Supporting Information

\title{
Resolvation-Based Damage to Metal-Organic Frameworks and Approaches to Mitigation
}

\author{
Ryan A. Dodson ${ }^{\dagger}$ and Adam J. Matzger ${ }^{*},+, \neq$ \\ ${ }^{\dagger}$ Department of Chemistry and ${ }^{\ddagger}$ Macromolecular Science and Engineering Program, \\ University of Michigan, 930 North University Avenue, Ann Arbor, Michigan 48109-1055, \\ United States
}

\section{Table of Contents:}

1. Reagents

2. Syntheses

3. Experimental details

4. Resolvation protocols

Tables S1-S8

5. Additional figures

Figures S1-S2: UiO-66 surface area comparisons and 2D-PXRD patterns

Figures S3-S6: Projections of 2D diffractograms along 2Ө-axes

Figures S7-S12: 2D-PXRD patterns for fragile MOFs solvated in DMF

6. BET surface area values

Tables S9-S12

7. Raw isotherm data

Tables S13-S22: UMCM-9

Tables S23-S30: IRMOF-3

Tables S31-S40: FJI-1

Tables S41-S48: UiO-66 


\section{Reagents}

Zinc perchlorate hexahydrate (Alfa Aesar, reagent grade), zirconium(IV) chloride (Strem, sublimed grade), 1,4-benzenedicarboxylic acid $\left(\mathrm{H}_{2} \mathrm{bdc}\right.$, Fisher Scientific, 98\%), 2aminoterephthalic acid $\left(\mathrm{H}_{2}\right.$ bdc- $\mathrm{NH}_{2}$, Sigma-Aldrich, 99\%), 2,6-naphthalenedicarboxylic acid $\left(\mathrm{H}_{2} \mathrm{ndc}\right.$, TCl America, 98\%), 4,4'-biphenyldicarboxylic acid $\left(\mathrm{H}_{2} \mathrm{bpdc}\right.$, Acros Organics, 98\%), 1,3,5-tri(4-carboxyphenyl)benzene ( $\mathrm{H}_{3}$ btb, Alfa Aesar, 97\%), 4,4'-bipyridine (4,4'-bpy, Acros Organics, 98\%), ethanol (Decon Laboratories, anhydrous), hydrochloric acid (Fisher Scientific, certified ACS plus), and fluoroboric acid (Acros Organics, 50 wt\% solution in water) were used as received. Zinc nitrate hexahydrate (Fisher Scientific, ACS grade) was partially dehydrated by room temperature evacuation ( $\sim 16 \mathrm{hr},<0.01 \mathrm{Torr})$ to yield zinc nitrate tetrahydrate (verified by thermogravimetric analysis). Hexane (Fisher Scientific, anhydrous), dichloromethane $\left(\mathrm{CH}_{2} \mathrm{Cl}_{2}\right.$, Fisher scientific, HPLC grade), tetrahydrofuran (THF, Fisher Scientific, HPLC grade), N,Ndimethylformamide (DMF, Fisher Scientific, 99.5\%), and dimethyl sulfoxide (DMSO, Fisher Scientific, HPLC grade) were stored over activated $4 \AA$ molecular sieves to minimize water content. The dryness of these solvents was monitored by Karl Fischer titration $\left(<10 \mathrm{ppm} \mathrm{H}_{2} \mathrm{O}\right.$ ). $N, N$-Diethylformamide (DEF, TCl America, >99.0\%) was purified by storage on activated charcoal for $\sim 1$ month followed by removal of impurities via silica gel column. Deionized water was used without further purification.

\section{Syntheses}

Each MOF synthesis was preceded by dissolution of reagents by sonication and was carried out in a $20 \mathrm{~mL}$ scintillation vial sealed with a PTFE-lined cap, and the washing procedures for each MOF were identical. The as-synthesized crystals were removed from the reaction vial and washed with DMF $(3 \times 10 \mathrm{~mL})$ and then washed $(3 \times 10 \mathrm{~mL})$ with the desired activation solvent as described below. Procedures specific to individual MOFs are included below:

Synthesis of UMCM-9. $\mathrm{H}_{2}$ ndc (29.1 mg, $\left.0.135 \mathrm{mmol}\right), \mathrm{H}_{2} \mathrm{bpdc}(36.8 \mathrm{mg}, 0.152 \mathrm{mmol}$ ), and $\mathrm{Zn}\left(\mathrm{NO}_{2}\right)_{3} \cdot 4 \mathrm{H}_{2} \mathrm{O}(216.0 \mathrm{mg}, 0.826 \mathrm{mmol})$ were dissolved in DEF $(6.7 \mathrm{~mL})$ and NMP $(13.3 \mathrm{~mL})$. The reaction was performed at $85^{\circ} \mathrm{C}$ for 7 days. Prior to activation, UMCM- 9 was washed $3 \times$ with DMF, $3 \times$ with $\mathrm{CH}_{2} \mathrm{Cl}_{2}$, then $3 \times$ with hexane. Activation was performed by room temperature evacuation of hexane from the MOF ( 0.001 Torr) for $16 \mathrm{hrs}$.

Synthesis of IRMOF-3. $\mathrm{H}_{2} \mathrm{bdc}-\mathrm{NH}_{2}(75.0 \mathrm{mg}, 0.414 \mathrm{mmol})$ and $\mathrm{Zn}\left(\mathrm{NO}_{2}\right)_{3} \cdot 4 \mathrm{H}_{2} \mathrm{O}(300.0 \mathrm{mg}, 1.147$ $\mathrm{mmol})$ were dissolved in DMF $(10 \mathrm{~mL})$. The reaction was performed at $100{ }^{\circ} \mathrm{C}$ for 16 hours. Prior to activation, IRMOF-3 was washed $3 \times$ with DMF, then $3 \times$ with $\mathrm{CH}_{2} \mathrm{Cl}_{2}$. Activation was performed by room temperature evacuation of $\mathrm{CH}_{2} \mathrm{Cl}_{2}$ from the MOF ( 0.001 Torr) for $16 \mathrm{hrs}$.

Synthesis of FJl-1. $\mathrm{H}_{3}$ btb (175.0 mg, $\left.0.399 \mathrm{mmol}\right), 4,4$ '-bpy $(47.0 \mathrm{mg}, 0.301 \mathrm{mmol})$ and $\mathrm{Zn}\left(\mathrm{ClO}_{4}\right)_{2} \cdot 6 \mathrm{H}_{2} \mathrm{O}(223.0 \mathrm{mg}, 0.599 \mathrm{mmol})$ were dissolved in DMF $(10 \mathrm{~mL})$ with fluoroboric acid $(0.1 \mathrm{~mL})$. The reaction was performed at $85{ }^{\circ} \mathrm{C}$ for 72 hours. Prior to activation, FJI-1 was washed $3 \times$ with DMF. Activation was performed by flowing $\mathrm{scCO}_{2}$ treatment for $4 \mathrm{hrs}$ (backpressure regulator set at $100 \mathrm{bar} ; 1 \mathrm{hr}$ at $2 \mathrm{~mL} \mathrm{~min}^{-1}$ at ambient temperature, $1 \mathrm{hr}$ at $2 \mathrm{~mL}$ $\min ^{-1}$ at $55^{\circ} \mathrm{C}, 2 \mathrm{hrs}$ at $1 \mathrm{~mL} \min ^{-1}$ at $55^{\circ} \mathrm{C}$ ). 
Synthesis of UiO-66. $\mathrm{H}_{2} \mathrm{bdc}(123.0 \mathrm{mg}, 0.740 \mathrm{mmol})$ and $\mathrm{ZrCl}_{4}(125.0 \mathrm{mg}, 0.536 \mathrm{mmol})$ were dissolved in DMF $(15 \mathrm{~mL})$ with hydrochloric acid $(1 \mathrm{~mL})$. The reaction was performed at $85^{\circ} \mathrm{C}$ for 16 hours. Prior to activation, UiO-66 was washed $3 \times$ with DMF, then $3 \times$ with EtOH. Activation was performed by evacuation of $\mathrm{EtOH}$ from the MOF ( 0.001 Torr) for 16 hrs at 120 ${ }^{\circ} \mathrm{C}$.

\section{Experimental details}

PXRD patterns were collected in $1 \mathrm{~mm}$ diameter Kapton capillaries using a Rigaku SmartLab $\mathrm{X}$-ray diffractometer ( $\mathrm{Cu} \mathrm{K}$ radiation) operating in point focus mode outfitted with a Pilatus $2 \mathrm{D}$ detector. Patterns were collected between $3-40^{\circ} 2 \theta$, with 8-minute exposure times. Due to the height error associated with sample-filled $1 \mathrm{~mm}$ capillaries, data were refined prior to analysis by adjusting the beam center position such that the primary peak of the pristine sample aligned with the known value for each MOF. This correction was applied uniformly across each set of MOFs to ensure that any peak shifts would be apparent. Sample surface areas were calculated using the BET method from $\mathrm{N}_{2}$ sorption isotherms measured on a Quantachrome NOVA 4200 gas sorption analyzer.

\section{Resolvation protocols}

Generally, resolvation was performed by direct submersion of activated, pristine MOF samples $(\sim 30 \mathrm{mg})$ in the solvent under examination $(2 \mathrm{~mL})$. Following a 20 -minute equilibration period (after which samples sank to the bottom of the vial and bubbles were no longer observed to emerge from the MOFs), the solvent was decanted and replaced with activation solvent appropriate for the MOF ( $3 \times$ washes $/ 4 \mathrm{~mL} / 20 \mathrm{~min}$ ). After equilibration with the third wash of the activation solvent, MOFs were re-activated as described above. Deviations were made from this protocol in two cases:

1. UMCM-9 was activated from hexane, which is immiscible with DMF and DMSO. When UMCM-9 was resolvated with these two solvents, the samples were washed $3 \times$ with $\mathrm{CH}_{2} \mathrm{Cl}_{2}$ prior to the $3 \times$ washes with hexane.

2. $\mathrm{FJI}-1$ was re-activated via flowing $\mathrm{scCO}_{2}$ directly from the resolvation solvent.

Reverse solvent exchange (RSE) was performed in a similar fashion to resolvation. Rather than direct submersion in the solvent to be studied, the MOFs were first resolvated with their activation solvent (or hexane, in the case of FJI-1). For UMCM-9 RSE into DMF or DMSO, this therefore required 6 washing steps with $\mathrm{CH}_{2} \mathrm{Cl}_{2}$, in order to facilitate the hexane $\rightarrow$ DMF/DMSO exchange as well as the DMF/DMSO $\rightarrow$ hexane exchange. Similarly, FJI-1 RSE into DMF or DMSO required 3 washing steps with $\mathrm{CH}_{2} \mathrm{Cl}_{2}$, in order to facilitate the hexane $\rightarrow$ DMF/DMSO exchange. The exact steps for each resolvation/RSE treatment are given in Tables S1-S8.

Table S1. UMCM-9 resolvation washing steps.

\begin{tabular}{|l|l|l|l|l|l|}
\hline $\begin{array}{l}\text { Wash } \\
\#\end{array}$ & $\begin{array}{l}\text { Hexane } \\
\text { resolvation }\end{array}$ & $\begin{array}{l}\mathrm{CH}_{2} \mathrm{Cl}_{2} \\
\text { resolvation }\end{array}$ & $\begin{array}{l}\text { THF } \\
\text { resolvation }\end{array}$ & $\begin{array}{l}\mathrm{DMF} \\
\text { resolvation }\end{array}$ & $\begin{array}{l}\mathrm{DMSO} \\
\text { resolvation }\end{array}$ \\
\hline 1 & Hexane & $\mathrm{CH}_{2} \mathrm{Cl}_{2}$ & THF & $\mathrm{DMF}$ & $\mathrm{DMSO}$ \\
\hline 2 & & $\mathrm{Hexane}$ & Hexane & $\mathrm{CH}_{2} \mathrm{Cl}_{2}$ & $\mathrm{CH}_{2} \mathrm{Cl}_{2}$ \\
\hline 3 & & Hexane & Hexane & $\mathrm{CH}_{2} \mathrm{Cl}_{2}$ & $\mathrm{CH}_{2} \mathrm{Cl}_{2}$ \\
\hline 4 & & Hexane & Hexane & $\mathrm{CH}_{2} \mathrm{Cl}_{2}$ & $\mathrm{CH}_{2} \mathrm{Cl}_{2}$ \\
\hline
\end{tabular}




\begin{tabular}{|l|l|l|l|l|l|}
\hline 5 & & & & Hexane & Hexane \\
\hline 6 & & & & Hexane & Hexane \\
\hline 7 & & & & Hexane & Hexane \\
\hline
\end{tabular}

Table S2. UMCM-9 RSE washing steps.

\begin{tabular}{|c|c|c|c|c|}
\hline $\begin{array}{l}\text { Wash } \\
\#\end{array}$ & $\begin{array}{l}\mathrm{CH}_{2} \mathrm{Cl}_{2} \\
\mathrm{RSE}\end{array}$ & $\begin{array}{l}\text { THF } \\
\text { RSE }\end{array}$ & $\begin{array}{l}\text { DMF } \\
\text { RSE } \\
\end{array}$ & $\begin{array}{l}\text { DMSO } \\
\text { RSE }\end{array}$ \\
\hline 1 & Hexane & Hexane & Hexane & Hexane \\
\hline 2 & $\mathrm{CH}_{2} \mathrm{Cl}_{2}$ & THF & $\mathrm{CH}_{2} \mathrm{Cl}_{2}$ & $\mathrm{CH}_{2} \mathrm{Cl}_{2}$ \\
\hline 3 & $\mathrm{CH}_{2} \mathrm{Cl}_{2}$ & THF & $\mathrm{CH}_{2} \mathrm{Cl}_{2}$ & $\mathrm{CH}_{2} \mathrm{Cl}_{2}$ \\
\hline 4 & $\mathrm{CH}_{2} \mathrm{Cl}_{2}$ & THF & $\mathrm{CH}_{2} \mathrm{Cl}_{2}$ & $\mathrm{CH}_{2} \mathrm{Cl}_{2}$ \\
\hline 5 & Hexane & Hexane & DMF & DMSO \\
\hline 6 & Hexane & Hexane & DMF & DMSO \\
\hline 7 & Hexane & Hexane & DMF & DMSO \\
\hline 8 & & & $\mathrm{CH}_{2} \mathrm{Cl}_{2}$ & $\mathrm{CH}_{2} \mathrm{Cl}_{2}$ \\
\hline 9 & & & $\mathrm{CH}_{2} \mathrm{Cl}_{2}$ & $\mathrm{CH}_{2} \mathrm{Cl}_{2}$ \\
\hline 10 & & & $\mathrm{CH}_{2} \mathrm{Cl}_{2}$ & $\mathrm{CH}_{2} \mathrm{Cl}_{2}$ \\
\hline 11 & & & Hexane & Hexane \\
\hline 12 & & & Hexane & Hexane \\
\hline 13 & & & Hexane & Hexane \\
\hline
\end{tabular}

Table S3. IRMOF-3 resolvation washing steps.

\begin{tabular}{|l|l|l|l|l|}
\hline $\begin{array}{l}\text { Wash } \\
\#\end{array}$ & $\begin{array}{l}\mathrm{CH}_{2} \mathrm{Cl}_{2} \\
\text { resolvation }\end{array}$ & $\begin{array}{l}\text { THF } \\
\text { resolvation }\end{array}$ & $\begin{array}{l}\text { DMF } \\
\text { resolvation }\end{array}$ & $\begin{array}{l}\text { DMSO } \\
\text { resolvation }\end{array}$ \\
\hline 1 & $\mathrm{CH}_{2} \mathrm{Cl}_{2}$ & THF & $\mathrm{DMF}$ & $\mathrm{DMSO}$ \\
\hline 2 & & $\mathrm{CH}_{2} \mathrm{Cl}_{2}$ & $\mathrm{CH}_{2} \mathrm{Cl}_{2}$ & $\mathrm{CH}_{2} \mathrm{Cl}_{2}$ \\
\hline 3 & & $\mathrm{CH}_{2} \mathrm{Cl}_{2}$ & $\mathrm{CH}_{2} \mathrm{Cl}_{2}$ & $\mathrm{CH}_{2} \mathrm{Cl}_{2}$ \\
\hline 4 & & $\mathrm{CH}_{2} \mathrm{Cl}_{2}$ & $\mathrm{CH}_{2} \mathrm{Cl}_{2}$ & $\mathrm{CH}_{2} \mathrm{Cl}_{2}$ \\
\hline
\end{tabular}

Table S4. IRMOF-3 RSE washing steps.

\begin{tabular}{|l|l|l|l|}
\hline $\begin{array}{l}\text { Wash } \\
\#\end{array}$ & $\begin{array}{l}\text { THF } \\
\text { RSE }\end{array}$ & $\begin{array}{l}\text { DMF } \\
\text { RSE }\end{array}$ & $\begin{array}{l}\text { DMSO } \\
\text { RSE }\end{array}$ \\
\hline 1 & $\mathrm{CH}_{2} \mathrm{Cl}_{2}$ & $\mathrm{CH}_{2} \mathrm{Cl}_{2}$ & $\mathrm{CH}_{2} \mathrm{Cl}_{2}$ \\
\hline 2 & $\mathrm{THF}$ & $\mathrm{DMF}$ & $\mathrm{DMSO}$ \\
\hline 3 & $\mathrm{THF}$ & $\mathrm{DMF}$ & $\mathrm{DMSO}$ \\
\hline 4 & $\mathrm{THF}$ & $\mathrm{DMF}$ & $\mathrm{DMSO}^{-}$ \\
\hline 5 & $\mathrm{CH}_{2} \mathrm{Cl}_{2}$ & $\mathrm{CH}_{2} \mathrm{Cl}_{2}$ & $\mathrm{CH}_{2} \mathrm{Cl}_{2}$ \\
\hline 6 & $\mathrm{CH}_{2} \mathrm{Cl}_{2}$ & $\mathrm{CH}_{2} \mathrm{Cl}_{2}$ & $\mathrm{CH}_{2} \mathrm{Cl}_{2}$ \\
\hline 7 & $\mathrm{CH}_{2} \mathrm{Cl}_{2}$ & $\mathrm{CH}_{2} \mathrm{Cl}_{2}$ & $\mathrm{CH}_{2} \mathrm{Cl}_{2}$ \\
\hline
\end{tabular}

Table S5. FJI-1 resolvation washing steps.

\begin{tabular}{|l|l|l|l|l|l|}
\hline $\begin{array}{l}\text { Wash } \\
\#\end{array}$ & $\begin{array}{l}\text { Hexane } \\
\text { resolvation }\end{array}$ & $\begin{array}{l}\mathrm{CH}_{2} \mathrm{Cl}_{2} \\
\text { resolvation }\end{array}$ & $\begin{array}{l}\text { THF } \\
\text { resolvation }\end{array}$ & $\begin{array}{l}\text { DMF } \\
\text { resolvation }\end{array}$ & $\begin{array}{l}\text { DMSO } \\
\text { resolvation }\end{array}$ \\
\hline 1 & Hexane & $\mathrm{CH}_{2} \mathrm{Cl}_{2}$ & THF & DMF & DMSO \\
\hline
\end{tabular}

Table S6. FJI-1 RSE washing steps.

\begin{tabular}{|l|l|l|l|l|}
\hline $\begin{array}{l}\text { Wash } \\
\#\end{array}$ & $\mathrm{CH}_{2} \mathrm{Cl}_{2}$ & THF & DMF & DMSO \\
\hline 1 & $\mathrm{HSE}$ & RSE & RSE & RSE \\
\hline 2 & $\mathrm{CH}_{2} \mathrm{Cl}_{2}$ & THF & $\mathrm{CH}_{2} \mathrm{Cl}_{2}$ & $\mathrm{CH}_{2} \mathrm{Cl}_{2}$ \\
\hline 3 & $\mathrm{CH}_{2} \mathrm{Cl}_{2}$ & THF & $\mathrm{CH}_{2} \mathrm{Cl}_{2}$ & $\mathrm{CH}_{2} \mathrm{Cl}_{2}$ \\
\hline 4 & $\mathrm{CH}_{2} \mathrm{Cl}_{2}$ & THF & $\mathrm{CH}_{2} \mathrm{Cl}_{2}$ & $\mathrm{CH}_{2} \mathrm{Cl}_{2}$ \\
\hline 5 & & & DMF & DMSO \\
\hline
\end{tabular}




\begin{tabular}{|l|l|l|l|l|}
\hline 6 & & & DMF & DMSO \\
\hline 7 & & & DMF & DMSO \\
\hline
\end{tabular}

Table S7. UiO-66 resolvation washing steps.

\begin{tabular}{|l|l|l|l|l|}
\hline $\begin{array}{l}\text { Wash } \\
\#\end{array}$ & $\begin{array}{l}\text { EtOH } \\
\text { resolvation }\end{array}$ & $\begin{array}{l}\text { DMF } \\
\text { resolvation }\end{array}$ & $\begin{array}{l}\text { DMSO } \\
\text { resolvation }\end{array}$ & $\begin{array}{l}\mathrm{H}_{2} \mathrm{O} \\
\text { resolvation }\end{array}$ \\
\hline 1 & EtOH & DMF & DMSO & $\mathrm{H}_{2} \mathrm{O}$ \\
\hline 2 & & $\mathrm{EtOH}$ & $\mathrm{EtOH}$ & $\mathrm{EtOH}$ \\
\hline 3 & & $\mathrm{EtOH}$ & $\mathrm{EtOH}$ & $\mathrm{EtOH}$ \\
\hline 4 & & $\mathrm{EtOH}$ & $\mathrm{EtOH}$ & $\mathrm{EtOH}$ \\
\hline
\end{tabular}

Table S8. UiO-66 RSE washing steps.

\begin{tabular}{|l|l|l|l|}
\hline $\begin{array}{l}\text { Wash } \\
\#\end{array}$ & $\begin{array}{l}\text { DMF } \\
\text { RSE }\end{array}$ & $\begin{array}{l}\text { DMSO } \\
\text { RSE }\end{array}$ & $\begin{array}{l}\mathrm{H}_{2} \mathrm{O} \\
\text { RSE }\end{array}$ \\
\hline 1 & EtOH & EtOH & EtOH \\
\hline 2 & DMF & DMSO & $\mathrm{H}_{2} \mathrm{O}$ \\
\hline 3 & DMF & DMSO & $\mathrm{H}_{2} \mathrm{O}$ \\
\hline 4 & DMF & DMSO & $\mathrm{H}_{2} \mathrm{O}$ \\
\hline 5 & EtOH & EtOH & EtOH \\
\hline 6 & EtOH & EtOH & EtOH \\
\hline 7 & $\mathrm{EtOH}$ & $\mathrm{EtOH}$ & $\mathrm{EtOH}$ \\
\hline
\end{tabular}




\section{Additional figures}

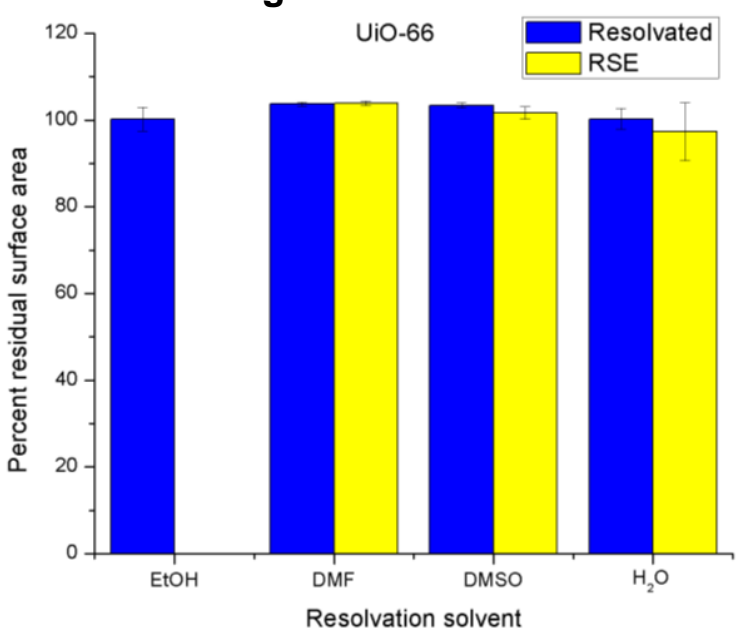

Figure S1. Percent residual surface area versus resolvation solvent for UiO-66. Blue: direct resolvation. Yellow: resolvation via RSE from hexane.

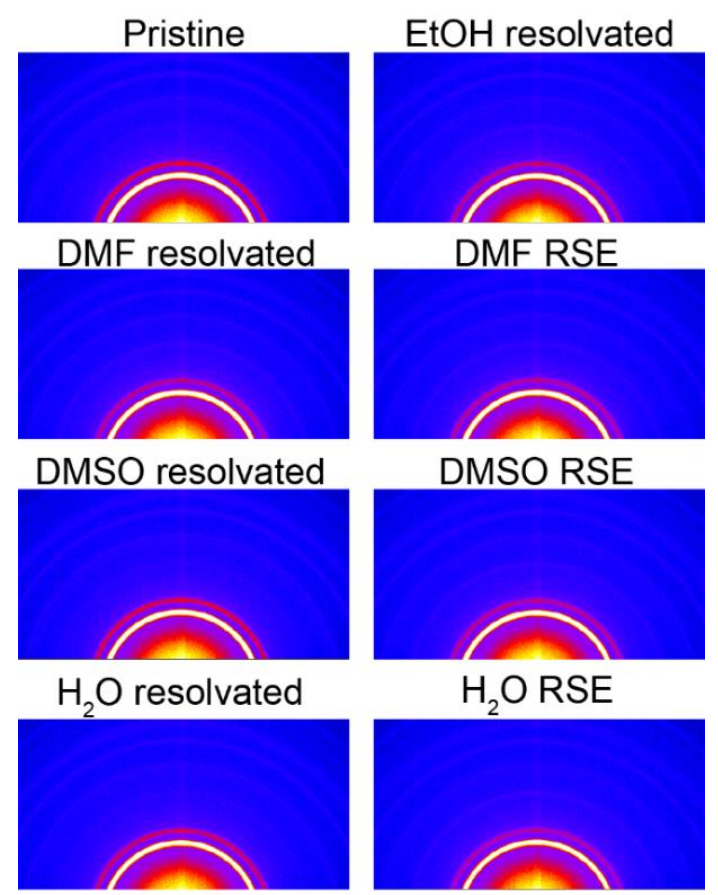

Figure S2. 2D-PXRD patterns for UiO-66 samples subjected to either direct resolvation or RSE treatment. Top left: pristine UiO-66 activated from EtOH. Top right: pristine UiO-66 activated from $\mathrm{EtOH}$, resolvated with $\mathrm{EtOH}$, and re-activated from EtOH. Left: UiO-66 resolvated with listed solvents, re-activated from $\mathrm{EtOH}$. Right: $\mathrm{UiO}-66$ resolvated with $\mathrm{EtOH}$, exchanged into listed solvents, and re-activated from $\mathrm{EtOH}$. UiO-66 was synthesized according to a common protocol which yields a microcrystalline powder; this synthesis was chosen with the goal of studying the behavior of a material that is most relevant to the field. However, the small crystallite size of this material makes it impossible to distinguish $\beta$-axis changes in the 2D-PXRD patterns of the material 


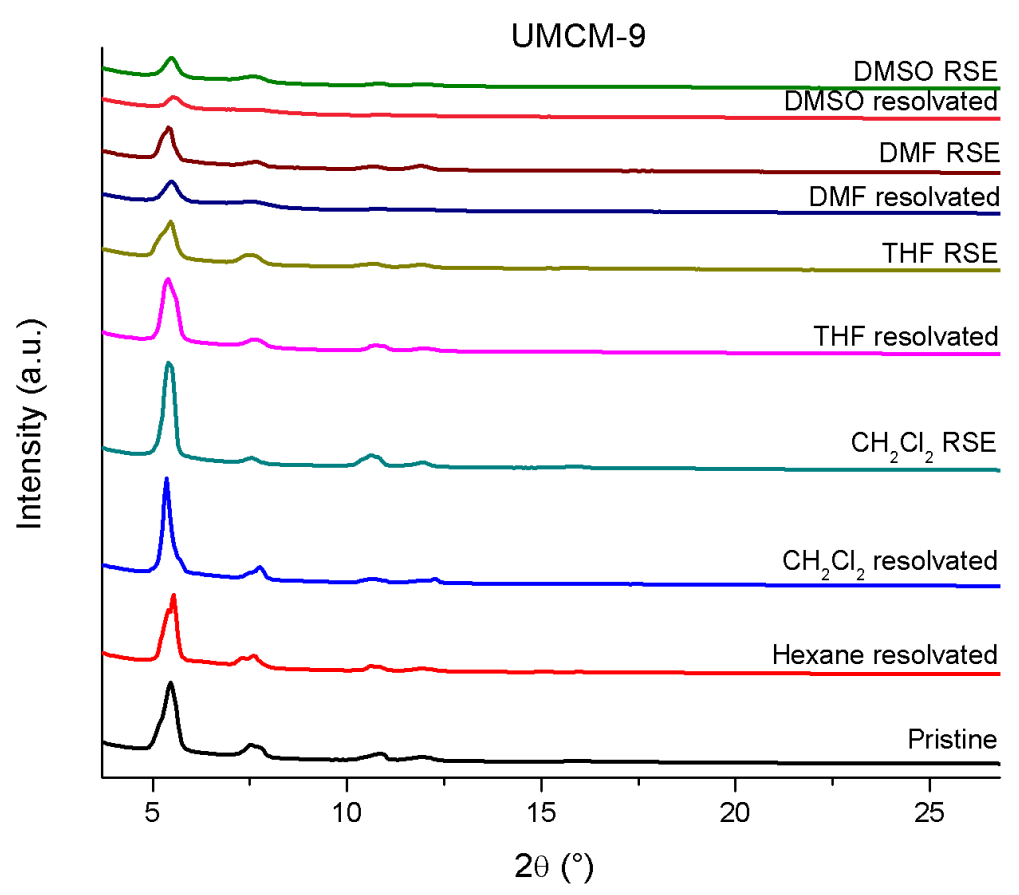

Figure S3. Diffracted X-ray intensity as a function of $2 \theta$ for the UMCM-9 resolvation and RSE samples studied. Greater retention of $10-15{ }^{\circ} 2 \theta$ peaks can be observed for high surface tension solvent (DMF, DMSO) RSE samples relative to the direct resolvation samples, consistent with better retention of sample crystallinity.

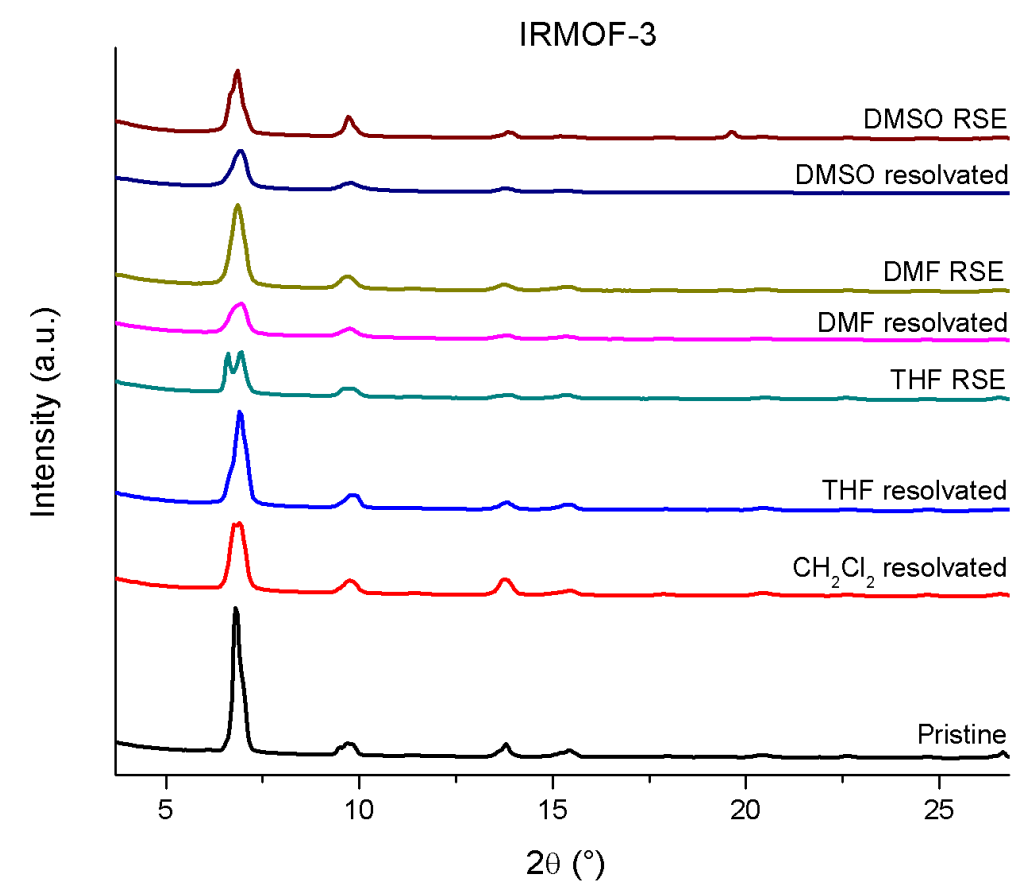

Figure S4. Diffracted X-ray intensity as a function of $2 \theta$ for the IRMOF-3 resolvation and RSE samples studied. Greater retention of $20-25{ }^{\circ} 2 \theta$ peaks can be observed for high surface tension solvent (DMF, DMSO) RSE samples relative to the direct resolvation samples, consistent with better retention of sample crystallinity. 


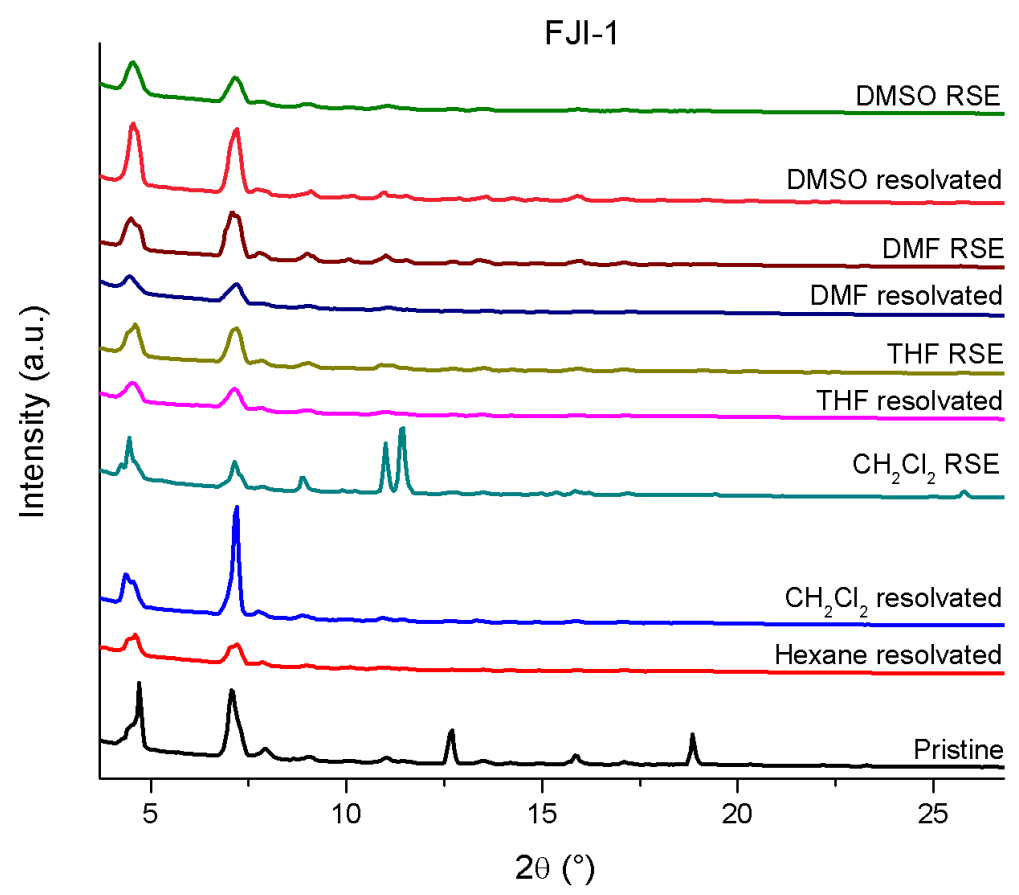

Figure S5. Diffracted X-ray intensity as a function of $2 \theta$ for the FJI-1 resolvation and RSE samples studied. Differences between peak intensities in samples which maintained near full surface area (e.g. pristine vs. $\mathrm{CH}_{2} \mathrm{Cl}_{2} \mathrm{RSE}$ ) are partially attributed to relatively high sample preferred orientation in prepared FJI-1 capillaries due to incomplete sample grinding, a requisite for determining changes in sample polycrystallinity via 2D-PXRD, as well as much larger initial crystal sizes $(\sim 5 \mathrm{~mm})$.

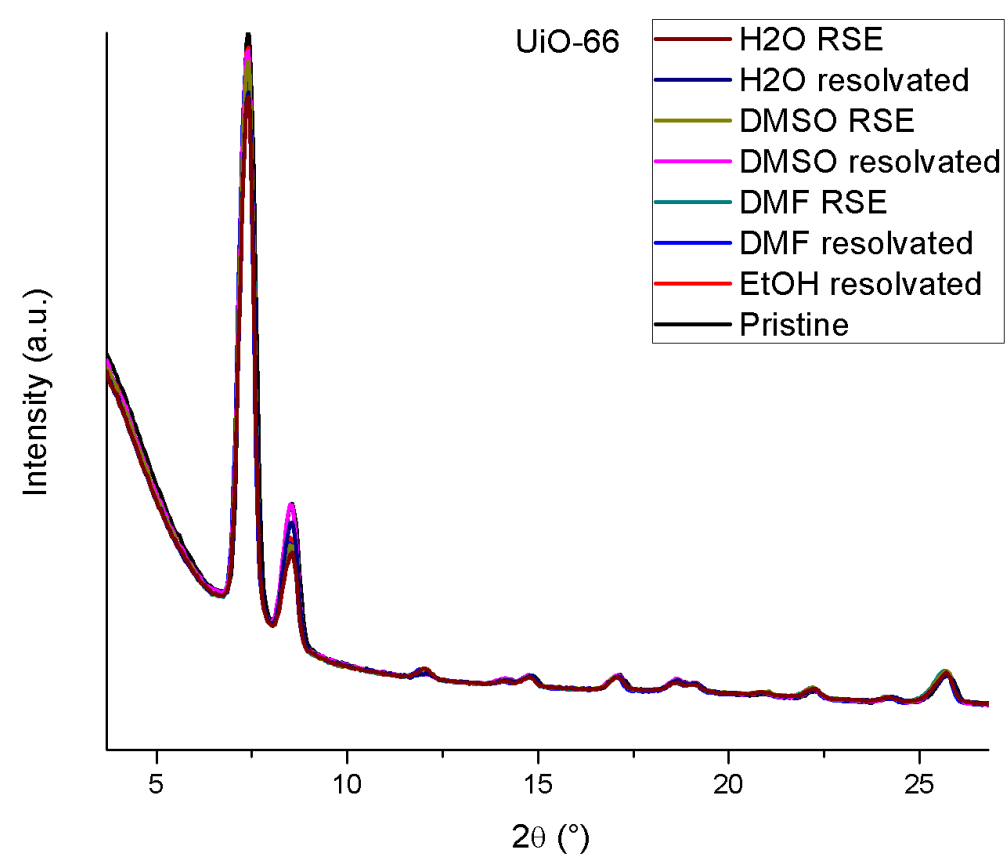

Figure S6. Diffracted X-ray intensity as a function of $2 \theta$ for the UiO-66 resolvation and RSE samples studied. Diffraction patterns are overlaid to demonstrate their strong similarity. The only observed changes in intensity are attributed to small differences in the quantity of sample packed into the capillaries. 


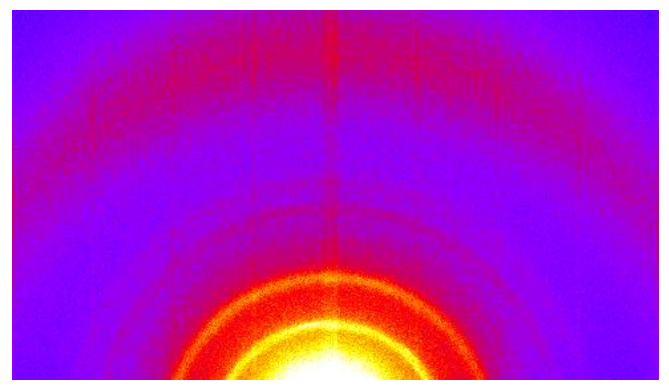

Figure S7. 2D-PXRD pattern for a DMF-solvated UMCM-9 sample subjected to direct DMF resolvation.

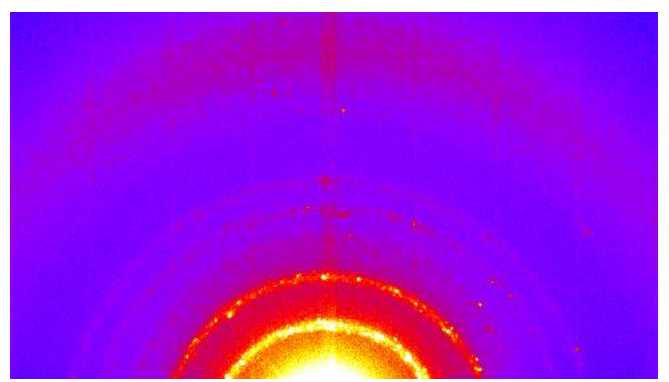

Figure S8. 2D-PXRD pattern for a DMF-solvated UMCM-9 sample subjected to DMF resolvation via RSE.

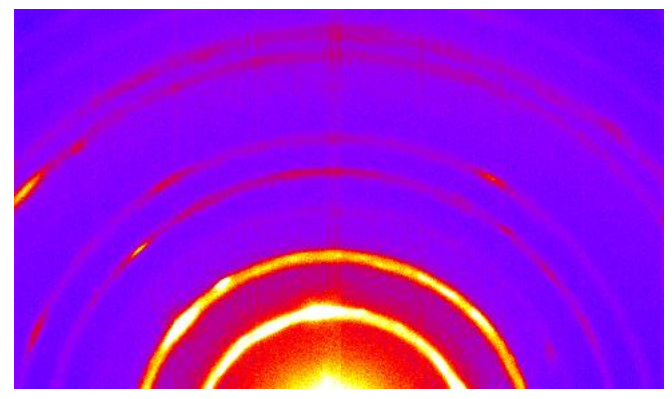

Figure S9. 2D-PXRD pattern for a DMF-solvated IRMOF-3 sample subjected to direct DMF resolvation.

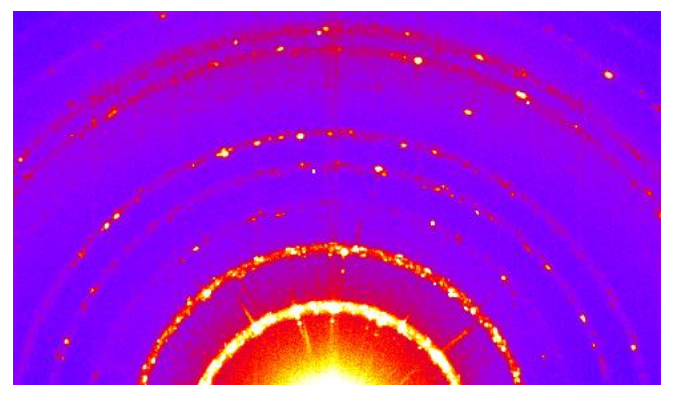

Figure S10. 2D-PXRD pattern for a DMF-solvated IRMOF-3 sample subjected to DMF resolvation via RSE. 


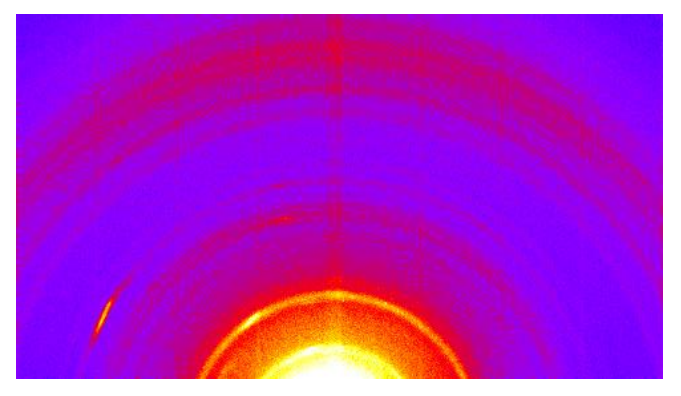

Figure S11. 2D-PXRD pattern for a DMF-solvated FJI-1 sample subjected to direct DMF resolvation.

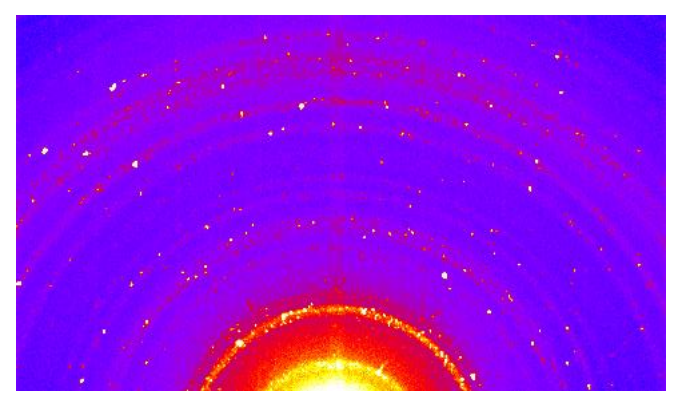

Figure S12. 2D-PXRD pattern for a DMF-solvated FJI-1 sample subjected to DMF resolvation via $R S E$. 


\section{BET surface area values}

Table S9. UMCM-9 BET surface area values. Pristine values associated with each sample are given in parentheses.

\begin{tabular}{|l|l|l|l|}
\hline $\begin{array}{l}\text { Resolvation } \\
\text { treatment }\end{array}$ & Trial 1 & Trial 2 & Trial 3 \\
\hline Hexane resolvated & $4871.445(4886.229)$ & $4597.616(4887.313)$ & $4959.377(4743.236)$ \\
\hline $\mathrm{CH}_{2} \mathrm{Cl}_{2}$ resolvated & $4839.069(4886.229)$ & $4723.449(4887.313)$ & $4748.670(4743.236)$ \\
\hline $\mathrm{THF}_{\text {resolvated }}$ & $3416.257(4886.229)$ & $3523.347(4887.313)$ & $3850.170(4743.236)$ \\
\hline DMF resolvated & $1168.332(4886.229)$ & $1018.117(4887.313)$ & $1014.082(4743.236)$ \\
\hline DMSO resolvated & $703.259(4886.229)$ & $582.618(4887.313)$ & $485.994(4743.236)$ \\
\hline $\mathrm{CH}_{2} \mathrm{Cl}_{2} \mathrm{RSE}$ & $4658.780(4743.236)$ & $4479.282(4639.937)$ & $4361.019(4639.937)$ \\
\hline $\mathrm{THF} \mathrm{RSE}$ & $4363.279(4743.236)$ & $4408.088(4639.937)$ & $4327.470(4639.937)$ \\
\hline DMF RSE & $4282.618(4743.236)$ & $3982.966(4639.937)$ & $3823.027(4639.937)$ \\
\hline DMSO RSE & $2744.301(4743.236)$ & $2710.679(4639.937)$ & $2230.496(4639.937)$ \\
\hline
\end{tabular}

Table S10. IRMOF-3 BET surface area values. Pristine values associated with each sample are given in parentheses.

\begin{tabular}{|l|l|l|l|}
\hline $\begin{array}{l}\text { Resolvation } \\
\text { treatment }\end{array}$ & Trial 1 & Trial 2 & Trial 3 \\
\hline $\mathrm{CH}_{2} \mathrm{Cl}_{2}$ resolvated & $2736.511(2846.728)$ & $2612.838(2758.320)$ & $2493.790(2723.582)$ \\
\hline THF resolvated & $2777.013(2846.728)$ & $2461.740(2758.320)$ & $2391.284(2723.582)$ \\
\hline DMF resolvated & $1564.853(2846.728)$ & $1526.210(2758.320)$ & $1650.651(2723.582)$ \\
\hline DMSO resolvated & $990.234(2846.728)$ & $1029.688(2758.320)$ & $1129.823(2723.582)$ \\
\hline THF RSE & $2451.428(2723.582)$ & $2461.384(2758.320)$ & $2625.397(2723.582)$ \\
\hline DMF RSE & $2220.588(2723.582)$ & $2358.293(2758.320)$ & $2395.788(2723.582)$ \\
\hline DMSO RSE & $2112.042(2723.582)$ & $2055.453(2758.320)$ & $2345.879(2723.582)$ \\
\hline
\end{tabular}

Table S11. FJI-1 BET surface area values. Pristine values associated with each sample are given in parentheses.

\begin{tabular}{|l|l|l|l|}
\hline $\begin{array}{l}\text { Resolvation } \\
\text { treatment }\end{array}$ & Trial 1 & Trial 2 & Trial 3 \\
\hline Hexane resolvated & $5141.839(4922.309)$ & $4309.985(4512.863)$ & $4617.747(4756.530)$ \\
\hline $\mathrm{CH}_{2} \mathrm{Cl}_{2}$ resolvated & $3051.493(4736.240)$ & $3156.182(4922.309)$ & $3524.140(4512.863)$ \\
\hline $\mathrm{THF}_{\mathrm{i} e s o l v a t e d}$ & $2167.888(4736.240)$ & $1646.980(4922.309)$ & $2474.469(4922.309)$ \\
\hline DMF resolvated & $1403.073(4736.240)$ & $1461.875(4922.309)$ & $2080.085(4756.530)$ \\
\hline DMSO resolvated & $3498.297(4922.309)$ & $1658.828(4922.309)$ & $3376.057(4756.530)$ \\
\hline $\mathrm{CH}_{2} \mathrm{Cl}_{2} \mathrm{RSE}$ & $4880.517(4922.309)$ & $4310.117(4922.309)$ & $4207.075(4512.863)$ \\
\hline THF RSE & $4344.884(4922.309)$ & $4672.283(4922.309)$ & $4822.559(4756.530)$ \\
\hline DMF RSE & $4690.209(4736.240)$ & $4624.500(4512.863)$ & $4954.712(4756.530)$ \\
\hline DMSO RSE & $4447.164(4922.309)$ & $3726.097(4512.863)$ & $4023.650(4756.530)$ \\
\hline
\end{tabular}


Table S12. UiO-66 BET surface area values. Pristine values associated with each sample are given in parentheses.

\begin{tabular}{|l|l|l|l|}
\hline $\begin{array}{l}\text { Resolvation } \\
\text { treatment }\end{array}$ & Trial 1 & Trial 2 & Trial 3 \\
\hline EtOH resolvated & $1535.346(1544.766)$ & $1504.327(1544.766)$ & $1604.913(1544.766)$ \\
\hline DMF resolvated & $1594.223(1544.766)$ & $1600.819(1544.766)$ & $1612.196(1544.766)$ \\
\hline DMSO resolvated & $1588.651(1544.766)$ & $1610.171(1544.766)$ & $1593.999(1544.766)$ \\
\hline $\mathrm{H}_{2} \mathrm{O}$ resolvated & $1505.456(1544.766)$ & $1543.641(1544.766)$ & $1597.201(1544.766)$ \\
\hline DMF RSE & $1706.754(1648.81)$ & $1599.434(1544.766)$ & $1616.194(1544.766)$ \\
\hline DMSO RSE & $1643.574(1648.81)$ & $1589.337(1544.766)$ & $1582.507(1544.766)$ \\
\hline $\mathrm{H}_{2} \mathrm{O}$ RSE & $1418.001(1648.81)$ & $1548.770(1544.766)$ & $1599.676(1544.766)$ \\
\hline
\end{tabular}

\section{Raw isotherm data}

\section{UMCM-9}

\section{Pristine samples}

Table S13. $\mathrm{N}_{2}$ isotherm data for pristine UMCM-9 samples used. Samples are labelled with their BET surface areas, given in parentheses.

\begin{tabular}{|l|l|l|l|l|l|}
\hline $\begin{array}{l}\text { Pristine } \\
(4886.229)\end{array}$ & $\begin{array}{l}\text { Pristine } \\
(4887.313)\end{array}$ & & $\begin{array}{l}\text { Pristine } \\
(4743.236)\end{array}$ & \\
\hline $\mathrm{P} / \mathrm{P}_{0}$ & $\begin{array}{l}\mathrm{N}_{2} \text { uptake } \\
\left(\mathrm{cc} \mathrm{g}^{-1}\right)\end{array}$ & $\mathrm{P} / \mathrm{P}_{0}$ & $\begin{array}{l}\mathrm{N}_{2} \text { uptake } \\
\left(\mathrm{cc} \mathrm{g}^{-1}\right)\end{array}$ & $\mathrm{P} / \mathrm{P}_{0}$ & $\begin{array}{l}\mathrm{N}_{2} \text { uptake } \\
\left(\mathrm{cc} \mathrm{g}^{-1}\right)\end{array}$ \\
\hline 0.001338 & 121.448 & 0.001667 & 131.7486 & 0.00176 & 132.4987 \\
\hline 0.002227 & 151.4731 & 0.002027 & 143.9085 & 0.002258 & 147.6816 \\
\hline 0.002434 & 158.4382 & 0.002215 & 149.4989 & 0.002489 & 154.3302 \\
\hline 0.002647 & 166.0549 & 0.002424 & 155.555 & 0.002756 & 161.6113 \\
\hline 0.002896 & 174.3455 & 0.002626 & 162.3281 & 0.00303 & 169.7847 \\
\hline 0.003174 & 183.2304 & 0.002864 & 169.4083 & 0.003355 & 178.2203 \\
\hline 0.003508 & 192.4386 & 0.003146 & 176.9069 & 0.00368 & 187.1035 \\
\hline 0.003886 & 202.9771 & 0.004163 & 206.4162 & 0.004033 & 196.6306 \\
\hline 0.004284 & 214.1292 & 0.005108 & 233.8595 & 0.005317 & 230.0782 \\
\hline 0.005117 & 238.6288 & 0.010238 & 357.4244 & 0.010426 & 342.4005 \\
\hline 0.011792 & 391.098 & 0.015267 & 441.095 & 0.015361 & 419.7723 \\
\hline 0.015869 & 455.8534 & 0.020375 & 512.339 & 0.02057 & 487.6923 \\
\hline 0.020402 & 518.9627 & 0.025021 & 584.1923 & 0.026695 & 574.925 \\
\hline 0.02611 & 610.403 & 0.030339 & 685.0366 & 0.030043 & 631.5458 \\
\hline 0.030849 & 703.6158 & 0.035418 & 803.4431 & 0.036175 & 754.4304 \\
\hline 0.035212 & 802.0498 & 0.040266 & 906.0776 & 0.040454 & 844.6796 \\
\hline 0.041317 & 936.8083 & 0.045252 & 992.517 & 0.045252 & 936.6118 \\
\hline 0.045765 & 1015.6574 & 0.051522 & 1059.2762 & 0.051341 & 1021.5338 \\
\hline 0.050547 & 1071.114 & 0.055403 & 1083.7811 & 0.056247 & 1061.4844 \\
\hline 0.055159 & 1103.3843 & 0.062228 & 1108.1532 & 0.060793 & 1082.6519 \\
\hline 0.060994 & 1127.6688 & 0.065879 & 1117.3331 & 0.0665 & 1099.8375 \\
\hline 0.065655 & 1140.7897 & 0.072466 & 1130.3423 & 0.07085 & 1109.5605 \\
\hline & & & & & \\
\hline
\end{tabular}




\begin{tabular}{|c|c|c|c|c|c|}
\hline 0.070508 & 1151.5048 & 0.077149 & 1137.6071 & 0.07637 & 1119.2653 \\
\hline 0.076244 & 1161.8573 & 0.082726 & 1145.1743 & 0.081644 & 1127.0826 \\
\hline 0.082727 & 1171.6665 & 0.088231 & 1151.8151 & 0.088556 & 1135.8774 \\
\hline 0.089417 & 1180.6207 & 0.095915 & 1159.8022 & 0.095453 & 1143.4412 \\
\hline 0.096974 & 1189.4729 & 0.100208 & 1164.0295 & 0.101918 & 1149.9186 \\
\hline 0.103236 & 1195.6401 & 0.145027 & 1195.4763 & 0.147905 & 1183.2412 \\
\hline 0.148019 & 1229.6373 & 0.197097 & 1221.1212 & 0.199578 & 1209.5826 \\
\hline 0.197805 & 1257.7205 & 0.251244 & 1241.0785 & 0.254419 & 1231.4049 \\
\hline 0.251071 & 1281.8148 & 0.305132 & 1258.3115 & 0.307917 & 1250.1376 \\
\hline 0.3064 & 1303.1857 & 0.359576 & 1273.9275 & 0.361127 & 1267.4528 \\
\hline 0.360477 & 1322.7224 & 0.412663 & 1287.5156 & 0.414424 & 1282.5343 \\
\hline 0.41153 & 1340.4115 & 0.466371 & 1301.6736 & 0.466212 & 1297.697 \\
\hline 0.46567 & 1357.9423 & 0.519025 & 1313.8212 & 0.516413 & 1311.5906 \\
\hline 0.517755 & 1374.2007 & 0.568462 & 1325.7159 & 0.569118 & 1325.322 \\
\hline 0.567982 & 1389.3346 & 0.619233 & 1337.0569 & 0.620402 & 1338.8806 \\
\hline 0.619034 & 1405.2467 & 0.670336 & 1348.6359 & 0.67105 & 1352.3676 \\
\hline 0.671403 & 1420.6644 & 0.721389 & 1360.2335 & 0.724138 & 1366.1984 \\
\hline 0.723616 & 1436.2327 & 0.773928 & 1372.2263 & 0.776965 & 1379.6136 \\
\hline 0.774149 & 1452.7494 & 0.82234 & 1382.9275 & 0.827072 & 1391.7034 \\
\hline 0.826254 & 1467.8889 & 0.873154 & 1394.8068 & 0.877447 & 1404.5657 \\
\hline 0.877029 & 1481.1389 & 0.922425 & 1407.1571 & 0.926732 & 1417.2649 \\
\hline 0.929946 & 1498.0494 & 0.971493 & 1418.876 & 0.975909 & 1430.0822 \\
\hline 0.975156 & 1513.5253 & 0.920585 & 1404.9961 & 0.923464 & 1413.4832 \\
\hline 0.922623 & 1494.8191 & 0.870636 & 1389.6353 & 0.869013 & 1396.0789 \\
\hline 0.870653 & 1477.1871 & 0.820096 & 1376.083 & 0.820731 & 1382.1304 \\
\hline 0.820675 & 1461.4185 & 0.770767 & 1364.5555 & 0.769627 & 1369.6467 \\
\hline 0.771238 & 1444.6801 & 0.719635 & 1353.2362 & 0.718214 & 1356.9501 \\
\hline 0.720548 & 1425.8158 & 0.672226 & 1342.7072 & 0.66973 & 1344.6071 \\
\hline 0.669496 & 1409.9517 & 0.622393 & 1331.7408 & 0.619608 & 1331.754 \\
\hline 0.622599 & 1395.52 & 0.572055 & 1321.2172 & 0.56839 & 1319.4004 \\
\hline 0.57043 & 1380.5144 & 0.526558 & 1311.3014 & 0.523931 & 1309.1381 \\
\hline 0.520594 & 1366.1115 & 0.471407 & 1300.083 & 0.474185 & 1297.1906 \\
\hline 0.47105 & 1351.2609 & 0.421069 & 1288.1792 & 0.420116 & 1282.8218 \\
\hline 0.423172 & 1336.4841 & 0.371993 & 1275.9668 & 0.372902 & 1269.5531 \\
\hline 0.372105 & 1320.5203 & 0.322131 & 1262.0203 & 0.320002 & 1252.4147 \\
\hline 0.321714 & 1303.703 & 0.271504 & 1245.4595 & 0.272853 & 1235.2401 \\
\hline 0.269772 & 1285.9353 & 0.222774 & 1228.7693 & 0.221562 & 1216.1527 \\
\hline 0.221652 & 1265.5547 & 0.175784 & 1209.3253 & 0.172905 & 1194.2622 \\
\hline \multirow[t]{4}{*}{0.173247} & 1242.52 & 0.123101 & 1179.8625 & 0.127494 & 1168.1511 \\
\hline & & 0.07694 & 1135.6134 & 0.074537 & 1114.1841 \\
\hline & & 0.029372 & 669.6445 & 0.029841 & 630.2878 \\
\hline & & 0.01922 & 496.0755 & 0.019184 & 470.5441 \\
\hline
\end{tabular}


Table S13 cont.

\begin{tabular}{|l|l|}
\hline $\begin{array}{l}\text { Pristine } \\
(4639.937)\end{array}$ & \\
\hline $\mathrm{P} / \mathrm{P}_{0}$ & $\begin{array}{l}\mathrm{N}_{2} \text { uptake } \\
\left(\mathrm{cc}^{-1}\right)\end{array}$ \\
\hline 0.001562 & 127.3639 \\
\hline 0.002145 & 147.0902 \\
\hline 0.002318 & 151.9786 \\
\hline 0.00252 & 157.3182 \\
\hline 0.002729 & 163.2837 \\
\hline 0.002959 & 169.4219 \\
\hline 0.003211 & 175.9539 \\
\hline 0.004219 & 201.8158 \\
\hline 0.005169 & 225.6803 \\
\hline 0.010864 & 342.5211 \\
\hline 0.01648 & 422.0432 \\
\hline 0.020972 & 477.8545 \\
\hline 0.02581 & 542.0718 \\
\hline 0.030526 & 614.5441 \\
\hline 0.035998 & 714.7361 \\
\hline 0.040843 & 805.2365 \\
\hline 0.045076 & 874.7419 \\
\hline 0.050677 & 944.2665 \\
\hline 0.055076 & 980.8764 \\
\hline 0.061189 & 1008.3234 \\
\hline 0.066221 & 1022.0355 \\
\hline 0.07026 & 1030.0784 \\
\hline 0.075465 & 1038.6124 \\
\hline 0.082744 & 1048.5162 \\
\hline 0.088821 & 1055.4229 \\
\hline 0.094271 & 1060.9299 \\
\hline 0.099937 & 1066.1846 \\
\hline 0.14597 & 1096.9416 \\
\hline 0.195438 & 1119.5641 \\
\hline 0.250191 & 1138.7201 \\
\hline 0.305397 & 1155.2965 \\
\hline 0.35989 & 1169.9951 \\
\hline 0.413656 & 1182.6891 \\
\hline 0.465996 & 1195.0244 \\
\hline 0.516076 & 1205.8235 \\
\hline 0.569201 & 1216.6313 \\
\hline 0.620239 & 1227.1788 \\
\hline 0.672075 & 1237.4052 \\
\hline 0.724653 & 1247.3395 \\
\hline
\end{tabular}




\begin{tabular}{|l|l|}
\hline 0.775748 & 1257.1487 \\
\hline 0.827117 & 1266.1825 \\
\hline 0.87886 & 1275.9797 \\
\hline 0.926802 & 1285.3129 \\
\hline 0.976039 & 1295.8752 \\
\hline 0.922518 & 1284.5959 \\
\hline 0.869177 & 1272.9109 \\
\hline 0.822185 & 1263.1678 \\
\hline 0.768038 & 1252.5958 \\
\hline 0.721594 & 1242.8612 \\
\hline 0.668728 & 1231.7773 \\
\hline 0.620692 & 1221.0613 \\
\hline 0.573953 & 1210.8254 \\
\hline 0.5237 & 1200.0452 \\
\hline 0.472764 & 1189.0085 \\
\hline 0.420589 & 1176.7872 \\
\hline 0.372309 & 1165.4791 \\
\hline 0.319868 & 1151.9561 \\
\hline 0.270515 & 1138.6896 \\
\hline 0.22109 & 1122.9647 \\
\hline 0.175704 & 1105.7451 \\
\hline 0.12165 & 1077.8994 \\
\hline 0.076322 & 1035.9994 \\
\hline 0.029885 & 607.1306 \\
\hline 0.019741 & 461.2468 \\
\hline & \\
\hline
\end{tabular}

Hexane resolvated samples

Table S14. $\mathrm{N}_{2}$ isotherm data for hexane resolvated UMCM-9 samples used.

\begin{tabular}{|c|c|c|c|c|c|}
\hline $\begin{array}{l}\text { Hexane } \\
\text { resolvation } 1\end{array}$ & & $\begin{array}{l}\text { Hexane } \\
\text { resolvation } 2\end{array}$ & & $\begin{array}{l}\text { Hexane } \\
\text { resolvation } 3\end{array}$ & \\
\hline $\mathrm{P} / \mathrm{P}_{0}$ & $\begin{array}{l}N_{2} \text { uptake } \\
\left(\mathrm{cc} \mathrm{g}^{-1}\right)\end{array}$ & $\mathrm{P} / \mathrm{P}_{0}$ & $\begin{array}{l}N_{2} \text { uptake } \\
\left(\mathrm{cc} \mathrm{g}^{-1}\right)\end{array}$ & $\mathrm{P} / \mathrm{P}_{0}$ & $\begin{array}{l}\mathrm{N}_{2} \text { uptake } \\
\left(\mathrm{cc} \mathrm{g}^{-1}\right)\end{array}$ \\
\hline 0.000854 & 102.8176 & 0.002183 & 142.8976 & 0.002304 & 151.7099 \\
\hline 0.001576 & 126.0468 & 0.002405 & 151.3999 & 0.002803 & 165.4619 \\
\hline 0.00204 & 138.7709 & 0.002676 & 160.6224 & 0.003331 & 180.0053 \\
\hline 0.002275 & 144.3276 & 0.003025 & 170.6378 & 0.003895 & 195.7011 \\
\hline 0.002504 & 150.3606 & 0.003375 & 181.0182 & 0.004387 & 212.185 \\
\hline 0.002739 & 156.94 & 0.003753 & 191.8324 & 0.005022 & 228.7104 \\
\hline 0.00299 & 164.1782 & 0.004167 & 203.4925 & 0.005664 & 245.8678 \\
\hline 0.003269 & 171.9515 & 0.004538 & 216.2154 & 0.006356 & 263.6201 \\
\hline 0.004271 & 201.7715 & 0.005373 & 239.6053 & 0.007097 & 281.6356 \\
\hline 0.005243 & 230.4159 & 0.010182 & 349.2979 & 0.010065 & 343.4959 \\
\hline 0.010611 & 363.1591 & 0.016083 & 441.8339 & 0.016128 & 438.8759 \\
\hline
\end{tabular}




\begin{tabular}{|c|c|c|c|c|c|}
\hline 0.016045 & 454.1748 & 0.020243 & 499.0471 & 0.020251 & 498.2961 \\
\hline 0.021398 & 530.5004 & 0.025417 & 578.5092 & 0.025986 & 581.5939 \\
\hline 0.025713 & 600.8777 & 0.030105 & 666.2097 & 0.030986 & 672.2932 \\
\hline 0.031037 & 706.9827 & 0.035999 & 793.3056 & 0.035551 & 767.4641 \\
\hline 0.035808 & 818.4945 & 0.041693 & 908.5762 & 0.040017 & 864.5951 \\
\hline 0.040624 & 928.0124 & 0.045025 & 959.7186 & 0.046158 & 983.8399 \\
\hline 0.045027 & 1008.0267 & 0.051547 & 1023.3932 & 0.05093 & 1048.8754 \\
\hline 0.051448 & 1081.0383 & 0.055129 & 1043.8704 & 0.056644 & 1096.3799 \\
\hline 0.055277 & 1105.5306 & 0.060609 & 1064.8115 & 0.061117 & 1118.5179 \\
\hline 0.062302 & 1131.7946 & 0.065718 & 1077.9871 & 0.067101 & 1138.3868 \\
\hline 0.065837 & 1140.9774 & 0.071234 & 1089.0662 & 0.073179 & 1153.3052 \\
\hline 0.072191 & 1153.6509 & 0.07802 & 1100.0099 & 0.080248 & 1167.5341 \\
\hline 0.076116 & 1160.0041 & 0.083749 & 1107.9565 & 0.085997 & 1177.2659 \\
\hline 0.081204 & 1167.3536 & 0.090557 & 1116.479 & 0.093472 & 1187.7766 \\
\hline 0.085799 & 1173.4076 & 0.098213 & 1124.9332 & 0.101597 & 1198.1506 \\
\hline 0.09194 & 1180.326 & 0.104856 & 1131.5743 & 0.107575 & 1205.2135 \\
\hline 0.098066 & 1186.5663 & 0.152757 & 1168.3876 & 0.158163 & 1252.6407 \\
\hline 0.147961 & 1221.6937 & 0.205474 & 1199.004 & 0.210284 & 1294.0204 \\
\hline 0.197458 & 1244.5177 & 0.259847 & 1225.3652 & 0.262784 & 1329.8661 \\
\hline 0.248369 & 1262.0239 & 0.313063 & 1247.9872 & 0.315455 & 1365.8298 \\
\hline 0.299685 & 1276.8252 & 0.365031 & 1269.3193 & 0.36702 & 1400.2544 \\
\hline 0.351325 & 1289.3098 & 0.416607 & 1289.4387 & 0.420233 & 1435.0269 \\
\hline 0.397781 & 1299.9332 & 0.469388 & 1309.6719 & 0.472006 & 1469.5714 \\
\hline 0.447631 & 1309.9238 & 0.521606 & 1328.7511 & 0.523521 & 1502.9099 \\
\hline 0.495457 & 1319.3661 & 0.571968 & 1349.2615 & 0.573039 & 1534.6053 \\
\hline 0.548592 & 1328.7982 & 0.622438 & 1368.2021 & 0.621858 & 1568.723 \\
\hline 0.596469 & 1337.2408 & 0.674556 & 1387.0991 & 0.673937 & 1603.4269 \\
\hline 0.64559 & 1345.708 & 0.728293 & 1406.1283 & 0.722671 & 1636.1168 \\
\hline 0.697429 & 1354.412 & 0.775509 & 1423.9126 & 0.773237 & 1673.5481 \\
\hline 0.746757 & 1360.7208 & 0.822732 & 1440.688 & 0.820573 & 1723.9731 \\
\hline 0.801003 & 1369.3944 & 0.873387 & 1460.229 & 0.868286 & 1770.1322 \\
\hline 0.850861 & 1377.776 & 0.923007 & 1478.8687 & 0.917619 & 1812.5326 \\
\hline 0.895314 & 1382.8784 & 0.974076 & 1498.9982 & 0.968264 & 1861.0565 \\
\hline 0.95258 & 1393.2075 & 0.918312 & 1474.7521 & 0.918268 & 1812.8109 \\
\hline 0.922876 & 1389.141 & 0.868342 & 1452.9658 & 0.869292 & 1772.6744 \\
\hline 0.869373 & 1384.1262 & 0.819892 & 1433.3514 & 0.81717 & 1727.0983 \\
\hline 0.814766 & 1379.0367 & 0.76726 & 1412.6122 & 0.768537 & 1688.8796 \\
\hline 0.765394 & 1371.5704 & 0.716427 & 1397.7942 & 0.719896 & 1655.6594 \\
\hline 0.729062 & 1367.7914 & 0.666385 & 1385.0977 & 0.667831 & 1619.7104 \\
\hline 0.666024 & 1359.8364 & 0.619377 & 1371.1547 & 0.617999 & 1583.8662 \\
\hline 0.61934 & 1353.0647 & 0.572867 & 1354.0813 & 0.571113 & 1550.6398 \\
\hline 0.570225 & 1344.2137 & 0.523468 & 1333.9296 & 0.523813 & 1519.3781 \\
\hline 0.516671 & 1335.9393 & 0.47135 & 1314.7498 & 0.470458 & 1484.2147 \\
\hline
\end{tabular}




\begin{tabular}{|l|l|l|l|l|l|}
\hline 0.470105 & 1326.3214 & 0.42091 & 1294.4144 & 0.418414 & 1447.3853 \\
\hline 0.421116 & 1316.218 & 0.370711 & 1274.2072 & 0.367513 & 1411.9352 \\
\hline 0.370308 & 1303.1281 & 0.31915 & 1253.03 & 0.317745 & 1377.6047 \\
\hline 0.319706 & 1288.5687 & 0.270436 & 1232.3655 & 0.26782 & 1342.5273 \\
\hline 0.269708 & 1273.9653 & 0.219274 & 1207.7245 & 0.220577 & 1309.527 \\
\hline 0.22407 & 1259.2203 & 0.172166 & 1181.9984 & 0.169889 & 1268.6157 \\
\hline & & 0.125992 & 1150.9061 & 0.121063 & 1223.2394 \\
\hline & & 0.07919 & 1103.1774 & 0.075732 & 1161.7749 \\
\hline & & 0.029141 & 655.7994 & 0.028804 & 635.9639 \\
\hline & & 0.018973 & 484.3526 & 0.019794 & 492.3433 \\
\hline
\end{tabular}

\section{$\mathrm{CH}_{2} \mathrm{Cl}_{2}$ resolvated samples}

Table S15. $\mathrm{N}_{2}$ isotherm data for $\mathrm{CH}_{2} \mathrm{Cl}_{2}$ resolvated UMCM-9 samples used.

\begin{tabular}{|l|l|l|l|l|l|}
\hline $\begin{array}{l}\mathrm{CH}_{2} \mathrm{Cl}_{2} \\
\text { resolvation 1 }\end{array}$ & $\begin{array}{l}\mathrm{CH}_{2} \mathrm{Cl}_{2} \\
\text { resolvation 2 }\end{array}$ & & $\begin{array}{l}\mathrm{CH}_{2} \mathrm{Cl}_{2} \\
\text { resolvation 3 }\end{array}$ & \\
\hline $\mathrm{P} / \mathrm{P}_{0}$ & $\left.\mathrm{Pc} \mathrm{g} \mathrm{P}_{0}\right)$ & $\begin{array}{l}\mathrm{N}_{2} \text { uptake } \\
\left(\mathrm{cc} \mathrm{g}^{-1}\right)\end{array}$ & $\mathrm{P} / \mathrm{P}_{0}$ & $\begin{array}{l}\mathrm{N}_{2} \text { uptake } \\
\left(\mathrm{cc} \mathrm{g}^{-1}\right)\end{array}$ \\
\hline 0.000862 & 102.8932 & 0.002319 & 152.0882 & 0.002789 & 158.6394 \\
\hline 0.001694 & 128.57 & 0.002447 & 157.6814 & 0.003053 & 165.4709 \\
\hline 0.002231 & 143.0098 & 0.002626 & 163.8124 & 0.003338 & 172.9295 \\
\hline 0.002452 & 149.0254 & 0.002847 & 170.5885 & 0.003545 & 181.5152 \\
\hline 0.002695 & 155.5455 & 0.003083 & 177.7026 & 0.00388 & 190.3519 \\
\hline 0.002938 & 162.6803 & 0.003354 & 185.3816 & 0.004244 & 199.6416 \\
\hline 0.003203 & 170.2931 & 0.003603 & 193.6359 & 0.004644 & 209.4696 \\
\hline 0.003498 & 178.5759 & 0.004217 & 213.3924 & 0.005072 & 220.3801 \\
\hline 0.004197 & 199.3481 & 0.005266 & 243.0668 & 0.005485 & 231.4812 \\
\hline 0.00528 & 231.0876 & 0.011431 & 381.3405 & 0.011577 & 353.7755 \\
\hline 0.011494 & 378.6632 & 0.016583 & 460.7737 & 0.015051 & 405.2699 \\
\hline 0.015044 & 437.5925 & 0.020265 & 512.0027 & 0.020272 & 476.0619 \\
\hline 0.021811 & 534.9156 & 0.025517 & 593.1702 & 0.02533 & 545.6231 \\
\hline 0.025183 & 589.8244 & 0.030754 & 695.7355 & 0.031314 & 643.8457 \\
\hline 0.030691 & 698.0439 & 0.035528 & 800.7624 & 0.035837 & 732.9795 \\
\hline 0.035389 & 807.6776 & 0.041008 & 916.1349 & 0.040987 & 839.4541 \\
\hline 0.040808 & 928.0407 & 0.045553 & 990.8456 & 0.045631 & 928.2318 \\
\hline 0.045072 & 1004.1877 & 0.051169 & 1049.9519 & 0.05011 & 993.4976 \\
\hline 0.052531 & 1082.5494 & 0.05555 & 1076.6555 & 0.055481 & 1042.4773 \\
\hline 0.055638 & 1100.979 & 0.060202 & 1094.3204 & 0.060275 & 1068.146 \\
\hline 0.060719 & 1120.7368 & 0.06661 & 1110.8649 & 0.066716 & 1089.0593 \\
\hline 0.066764 & 1136.9105 & 0.071134 & 1119.7275 & 0.071452 & 1100.0516 \\
\hline 0.070483 & 1144.2551 & 0.076471 & 1128.5031 & 0.077323 & 1110.8387 \\
\hline 0.075659 & 1153.3846 & 0.081238 & 1135.2599 & 0.082744 & 1118.9944 \\
\hline 0.080608 & 1160.7408 & 0.088074 & 1143.915 & 0.08992 & 1128.1615 \\
\hline 0.087839 & 1170.0844 & 0.094153 & 1150.6183 & 0.09696 & 1135.9308 \\
\hline & & & & & \\
\hline
\end{tabular}




\begin{tabular}{|c|c|c|c|c|c|}
\hline 0.09289 & 1175.73 & 0.101053 & 1157.3872 & 0.103131 & 1142.1672 \\
\hline 0.097993 & 1181.1528 & 0.146264 & 1189.9762 & 0.150052 & 1176.9056 \\
\hline 0.146827 & 1217.6013 & 0.196441 & 1215.3428 & 0.201368 & 1204.5005 \\
\hline 0.198982 & 1243.0977 & 0.252426 & 1237.1938 & 0.255145 & 1228.1053 \\
\hline 0.248435 & 1261.2 & 0.306006 & 1255.1195 & 0.30942 & 1248.9187 \\
\hline 0.299427 & 1277.7407 & 0.360215 & 1271.5609 & 0.361827 & 1268.2865 \\
\hline 0.351457 & 1292.1747 & 0.411826 & 1285.3213 & 0.414506 & 1285.4808 \\
\hline 0.395778 & 1304.3525 & 0.466127 & 1300.2947 & 0.465786 & 1302.8896 \\
\hline 0.450636 & 1317.7058 & 0.518473 & 1313.5702 & 0.517358 & 1319.4102 \\
\hline 0.500516 & 1329.2615 & 0.570984 & 1326.4771 & 0.569294 & 1335.465 \\
\hline 0.545823 & 1339.0441 & 0.622395 & 1339.1943 & 0.619447 & 1351.6321 \\
\hline 0.598751 & 1349.1234 & 0.673871 & 1351.3484 & 0.670292 & 1367.4854 \\
\hline 0.649434 & 1360.2971 & 0.725867 & 1363.0801 & 0.724183 & 1383.9773 \\
\hline 0.698482 & 1368.9886 & 0.777293 & 1374.9161 & 0.775092 & 1401.8612 \\
\hline 0.745998 & 1378.6843 & 0.829047 & 1386.0182 & 0.824082 & 1422.269 \\
\hline 0.795459 & 1386.8618 & 0.881136 & 1398.1121 & 0.873129 & 1440.8667 \\
\hline 0.854661 & 1396.4567 & 0.929101 & 1409.3427 & 0.92289 & 1459.2701 \\
\hline 0.89714 & 1406.2837 & 0.97795 & 1421.4518 & 0.973542 & 1477.8174 \\
\hline 0.945975 & 1413.1907 & 0.922215 & 1406.2854 & 0.920993 & 1458.5731 \\
\hline 0.928192 & 1413.4556 & 0.868891 & 1392.5129 & 0.86947 & 1440.4224 \\
\hline 0.866545 & 1405.6105 & 0.82149 & 1380.9882 & 0.818076 & 1423.2783 \\
\hline 0.826083 & 1398.7559 & 0.76776 & 1368.5099 & 0.770063 & 1409.1954 \\
\hline 0.771159 & 1391.7783 & 0.729677 & 1364.4794 & 0.719582 & 1393.9813 \\
\hline 0.723289 & 1385.6958 & 0.669183 & 1353.5685 & 0.668231 & 1377.5588 \\
\hline 0.666665 & 1374.3826 & 0.619591 & 1342.9594 & 0.619041 & 1361.2412 \\
\hline 0.61741 & 1365.8076 & 0.570727 & 1330.6841 & 0.570842 & 1345.4741 \\
\hline 0.570417 & 1357.2842 & 0.522819 & 1318.6428 & 0.52315 & 1330.2721 \\
\hline 0.517481 & 1347.2904 & 0.474319 & 1305.8749 & 0.471107 & 1313.1487 \\
\hline 0.478875 & 1338.4475 & 0.423093 & 1291.5664 & 0.419242 & 1294.573 \\
\hline 0.421712 & 1322.9772 & 0.371867 & 1277.3898 & 0.369524 & 1277.083 \\
\hline 0.367112 & 1306.4189 & 0.321134 & 1261.8463 & 0.320006 & 1258.5531 \\
\hline 0.317755 & 1290.8397 & 0.271941 & 1246.0698 & 0.27011 & 1239.7473 \\
\hline 0.273721 & 1275.2151 & 0.221964 & 1227.2643 & 0.221177 & 1218.0822 \\
\hline \multirow[t]{5}{*}{0.224003} & 1256.4882 & 0.175719 & 1206.9617 & 0.172928 & 1193.8359 \\
\hline & & 0.129538 & 1180.7417 & 0.126442 & 1164.1814 \\
\hline & & 0.076956 & 1130.4816 & 0.07335 & 1106.1719 \\
\hline & & 0.029463 & 677.6927 & 0.029467 & 616.5276 \\
\hline & & 0.019723 & 507.097 & 0.019259 & 464.2281 \\
\hline
\end{tabular}




\section{THF resolvated samples}

Table S16. $\mathrm{N}_{2}$ isotherm data for THF resolvated UMCM-9 samples used.

\begin{tabular}{|c|c|c|c|c|c|}
\hline $\begin{array}{l}\text { THF } \\
\text { resolvation } 1\end{array}$ & & $\begin{array}{l}\text { THF } \\
\text { resolvation } 2\end{array}$ & & $\begin{array}{l}\text { THF } \\
\text { resolvation } 3\end{array}$ & \\
\hline $\mathrm{P} / \mathrm{P}_{0}$ & $\begin{array}{l}\mathrm{N}_{2} \text { uptake } \\
\left(\mathrm{cc} \mathrm{g}^{-1}\right)\end{array}$ & $\mathrm{P} / \mathrm{P}_{0}$ & $\begin{array}{l}\mathrm{N}_{2} \text { uptake } \\
\left(\mathrm{cc} \mathrm{g}^{-1}\right)\end{array}$ & $\mathrm{P} / \mathrm{P}_{0}$ & $\begin{array}{l}\mathrm{N}_{2} \text { uptake } \\
\left(\mathrm{cc} \mathrm{g}^{-1}\right)\end{array}$ \\
\hline 0.001119 & 96.5602 & 0.002348 & 138.4489 & 0.002097 & 118.894 \\
\hline 0.001833 & 113.5284 & 0.002505 & 143.4323 & 0.002446 & 125.782 \\
\hline 0.00218 & 120.5519 & 0.002697 & 148.9138 & 0.002854 & 133.5293 \\
\hline 0.002555 & 128.189 & 0.002961 & 154.9165 & 0.003269 & 141.7052 \\
\hline 0.002945 & 136.3503 & 0.003218 & 161.2355 & 0.003713 & 150.4104 \\
\hline 0.003365 & 145.085 & 0.003518 & 168.0051 & 0.004201 & 159.7925 \\
\hline 0.003807 & 154.2796 & 0.00381 & 175.2662 & 0.004703 & 169.5387 \\
\hline 0.004278 & 164.256 & 0.004103 & 183.4794 & 0.00522 & 179.5252 \\
\hline 0.004757 & 174.7995 & 0.005409 & 211.2484 & 0.005817 & 190.7634 \\
\hline 0.005272 & 185.5421 & 0.01106 & 306.6071 & 0.011146 & 277.6443 \\
\hline 0.010191 & 271.1646 & 0.015263 & 357.0137 & 0.015274 & 326.7872 \\
\hline 0.015861 & 339.4452 & 0.021949 & 428.9156 & 0.020289 & 376.6397 \\
\hline 0.020647 & 388.1961 & 0.025909 & 477.418 & 0.025262 & 426.3637 \\
\hline 0.025124 & 438.4272 & 0.030569 & 543.4325 & 0.031537 & 500.585 \\
\hline 0.030396 & 509.9272 & 0.035114 & 612.2186 & 0.035825 & 559.9279 \\
\hline 0.036722 & 605.4405 & 0.040458 & 686.8381 & 0.040885 & 632.8437 \\
\hline 0.040661 & 661.3959 & 0.047194 & 753.489 & 0.045646 & 697.8566 \\
\hline 0.044991 & 706.5098 & 0.050769 & 775.9655 & 0.050203 & 747.3752 \\
\hline 0.050609 & 744.3038 & 0.056142 & 796.2482 & 0.055758 & 788.2574 \\
\hline 0.055771 & 764.3315 & 0.060024 & 805.8008 & 0.060657 & 809.3951 \\
\hline 0.063009 & 780.7942 & 0.067516 & 818.6036 & 0.065433 & 822.3434 \\
\hline 0.067832 & 788.5477 & 0.072154 & 824.6355 & 0.070078 & 830.8224 \\
\hline 0.072633 & 794.7545 & 0.078391 & 831.5286 & 0.075596 & 839.1374 \\
\hline 0.079157 & 801.7437 & 0.083521 & 836.4448 & 0.08332 & 848.1032 \\
\hline 0.083656 & 805.9816 & 0.0905 & 842.465 & 0.089938 & 854.1811 \\
\hline 0.088325 & 810.0668 & 0.09837 & 848.4166 & 0.096118 & 859.7447 \\
\hline 0.095578 & 815.6649 & 0.105085 & 852.9067 & 0.100872 & 863.2542 \\
\hline 0.099782 & 818.8013 & 0.151094 & 876.4299 & 0.146656 & 888.2487 \\
\hline 0.148653 & 844.6412 & 0.204247 & 895.7127 & 0.199058 & 906.5652 \\
\hline 0.199638 & 863.4519 & 0.257107 & 911.171 & 0.246924 & 920.4383 \\
\hline 0.247368 & 877.6379 & 0.31128 & 924.9681 & 0.295919 & 931.3466 \\
\hline 0.296106 & 889.5927 & 0.36496 & 937.5987 & 0.351276 & 943.2972 \\
\hline 0.352496 & 903.3121 & 0.417784 & 948.915 & 0.40073 & 951.9452 \\
\hline 0.399084 & 912.8175 & 0.468767 & 959.6913 & 0.448297 & 961.1887 \\
\hline 0.445481 & 923.4951 & 0.520029 & 970.4811 & 0.50134 & 969.5233 \\
\hline 0.504271 & 933.7878 & 0.570006 & 980.437 & 0.551019 & 977.3798 \\
\hline 0.546824 & 942.0367 & 0.623665 & 991.052 & 0.596759 & 983.1662 \\
\hline
\end{tabular}




\begin{tabular}{|l|l|l|l|l|l|}
\hline 0.601903 & 952.8238 & 0.674434 & 1000.5923 & 0.648848 & 992.3586 \\
\hline 0.647321 & 961.7704 & 0.727216 & 1010.6331 & 0.698534 & 1000.5761 \\
\hline 0.703445 & 972.6344 & 0.777856 & 1020.2665 & 0.751016 & 1005.8195 \\
\hline 0.750468 & 981.632 & 0.828354 & 1030.2831 & 0.79905 & 1012.0954 \\
\hline 0.800046 & 987.9322 & 0.880001 & 1040.344 & 0.847513 & 1021.9186 \\
\hline 0.847827 & 998.2308 & 0.929828 & 1048.7811 & 0.897862 & 1029.3704 \\
\hline 0.8996 & 1009.0509 & 0.9784 & 1058.3204 & 0.947177 & 1035.0281 \\
\hline 0.947786 & 1016.6848 & 0.922201 & 1046.507 & 0.926581 & 1035.7018 \\
\hline 0.92669 & 1016.52 & 0.868984 & 1035.5343 & 0.878832 & 1031.1727 \\
\hline 0.866045 & 1006.9503 & 0.819506 & 1024.7167 & 0.817141 & 1024.7407 \\
\hline 0.826842 & 1003.3141 & 0.76624 & 1015.6906 & 0.779015 & 1025.646 \\
\hline 0.766623 & 996.7147 & 0.72987 & 1012.5099 & 0.720272 & 1018.8304 \\
\hline 0.729423 & 990.5378 & 0.670296 & 1003.9662 & 0.666932 & 1015.1146 \\
\hline 0.67042 & 982.9404 & 0.620654 & 995.061 & 0.616226 & 1007.7674 \\
\hline 0.618117 & 973.5067 & 0.571169 & 984.7311 & 0.567464 & 1000.9124 \\
\hline 0.567494 & 966.5584 & 0.523226 & 975.08 & 0.519249 & 995.6926 \\
\hline 0.518674 & 955.7685 & 0.471793 & 963.9265 & 0.477592 & 987.8376 \\
\hline 0.478109 & 948.341 & 0.421388 & 953.1143 & 0.418289 & 976.0176 \\
\hline 0.421646 & 936.4407 & 0.371361 & 942.3337 & 0.37977 & 970.8312 \\
\hline 0.370212 & 922.0687 & 0.320377 & 929.9971 & 0.316012 & 955.7488 \\
\hline 0.321186 & 909.1228 & 0.269273 & 917.0856 & 0.267782 & 943.1533 \\
\hline 0.27247 & 896.8976 & 0.220252 & 902.7191 & 0.219945 & 928.129 \\
\hline 0.221566 & 878.2913 & 0.173828 & 887.3293 & 0.17392 & 911.0368 \\
\hline & & 0.12729 & 867.4968 & 0.123047 & 885.7618 \\
\hline & & 0.073282 & 828.319 & 0.076928 & 846.7618 \\
\hline & & 0.029955 & 542.927 & 0.028196 & 459.693 \\
\hline & & 0.018966 & 400.0949 & 0.019489 & 369.0791 \\
\hline
\end{tabular}

DMF resolvated samples

Table S17. $\mathrm{N}_{2}$ isotherm data for DMF resolvated UMCM-9 samples used.

\begin{tabular}{|l|l|l|l|l|l|}
\hline $\begin{array}{l}\text { DMF } \\
\text { resolvation 1 }\end{array}$ & $\begin{array}{l}\text { DMF } \\
\text { resolvation 2 }\end{array}$ & $\begin{array}{l}\text { DMF } \\
\text { resolvation 3 }\end{array}$ & \\
\hline $\mathrm{P}_{\mathrm{P}}$ & $\begin{array}{l}\mathrm{N}_{2} \text { uptake } \\
\left(\mathrm{cc} \mathrm{g}^{-1}\right)\end{array}$ & $\mathrm{P} / \mathrm{P}_{0}$ & $\begin{array}{l}\mathrm{N}_{2} \text { uptake } \\
\left(\mathrm{cc} \mathrm{g}^{-1}\right)\end{array}$ & $\mathrm{P} / \mathrm{P}_{0}$ & $\begin{array}{l}\mathrm{N}_{2} \text { uptake } \\
\left(\mathrm{cc} \mathrm{g}^{-1}\right)\end{array}$ \\
\hline 0.002128 & 89.6953 & 0.002212 & 77.7682 & 0.003888 & 73.7443 \\
\hline 0.002431 & 93.2838 & 0.002697 & 82.5872 & 0.004412 & 76.9833 \\
\hline 0.002864 & 97.0049 & 0.003304 & 87.5176 & 0.005023 & 80.4062 \\
\hline 0.003362 & 100.9949 & 0.004017 & 92.6359 & 0.005729 & 84.0529 \\
\hline 0.003947 & 105.3123 & 0.004774 & 97.6528 & 0.006465 & 87.8291 \\
\hline 0.004581 & 109.746 & 0.005566 & 102.6186 & 0.007265 & 91.8198 \\
\hline 0.005267 & 114.1105 & 0.006451 & 107.7039 & 0.008132 & 95.7403 \\
\hline 0.006003 & 118.6068 & 0.007271 & 113.2735 & 0.008998 & 99.6093 \\
\hline 0.00684 & 123.2605 & 0.008277 & 118.289 & 0.009923 & 103.3434 \\
\hline
\end{tabular}




\begin{tabular}{|c|c|c|c|c|c|}
\hline 0.010974 & 141.8924 & 0.010646 & 128.5363 & 0.012056 & 110.9315 \\
\hline 0.015353 & 156.7374 & 0.016112 & 147.1975 & 0.016693 & 124.8061 \\
\hline 0.021731 & 176.2013 & 0.021043 & 162.8644 & 0.020158 & 134.0575 \\
\hline 0.02518 & 187.9136 & 0.025716 & 179.0682 & 0.026252 & 150.6862 \\
\hline 0.030923 & 208.9781 & 0.030882 & 198.8399 & 0.030911 & 165.1534 \\
\hline 0.036428 & 229.9688 & 0.035385 & 216.6276 & 0.035745 & 181.5511 \\
\hline 0.040223 & 242.535 & 0.040551 & 233.8976 & 0.040426 & 197.2712 \\
\hline 0.045771 & 255.7691 & 0.046402 & 247.5531 & 0.046592 & 214.8041 \\
\hline 0.05075 & 263.4886 & 0.05119 & 254.8529 & 0.052606 & 226.9351 \\
\hline 0.057041 & 269.4146 & 0.058354 & 261.5247 & 0.057578 & 233.2891 \\
\hline 0.062892 & 273.1582 & 0.064783 & 265.6796 & 0.062099 & 237.055 \\
\hline 0.069883 & 276.53 & 0.071305 & 268.7529 & 0.069211 & 241.0115 \\
\hline 0.075663 & 278.9028 & 0.077306 & 271.248 & 0.074926 & 243.4167 \\
\hline 0.082113 & 281.1406 & 0.084113 & 273.6938 & 0.082425 & 246.2158 \\
\hline 0.086968 & 282.7378 & 0.089008 & 275.3486 & 0.088402 & 248.2411 \\
\hline 0.094219 & 284.9153 & 0.096044 & 277.6046 & 0.092122 & 249.3209 \\
\hline 0.102379 & 287.1859 & 0.104221 & 280.1453 & 0.098834 & 251.3832 \\
\hline 0.108115 & 288.8067 & 0.109452 & 281.742 & 0.103726 & 252.6233 \\
\hline 0.158295 & 299.4373 & 0.163931 & 294.5049 & 0.14654 & 261.0122 \\
\hline 0.212912 & 308.8545 & 0.214629 & 305.6728 & 0.19528 & 268.1155 \\
\hline 0.265479 & 318.2703 & 0.268252 & 315.7811 & 0.250441 & 275.4769 \\
\hline 0.318134 & 327.232 & 0.321162 & 326.3183 & 0.301212 & 281.1794 \\
\hline 0.36858 & 336.2042 & 0.370383 & 335.8712 & 0.349937 & 287.5742 \\
\hline 0.4178 & 344.6321 & 0.420082 & 345.5601 & 0.399863 & 292.9127 \\
\hline 0.46847 & 352.0472 & 0.470394 & 355.8271 & 0.447286 & 299.3399 \\
\hline 0.519682 & 359.8893 & 0.5207 & 365.6162 & 0.496717 & 304.2738 \\
\hline 0.569811 & 368.3905 & 0.573574 & 376.3062 & 0.551295 & 309.8639 \\
\hline 0.620582 & 376.9749 & 0.623943 & 387.0473 & 0.597589 & 315.2426 \\
\hline 0.672674 & 386.3687 & 0.672286 & 396.9943 & 0.647254 & 320.2307 \\
\hline 0.72496 & 395.9441 & 0.72596 & 407.8312 & 0.698221 & 325.324 \\
\hline 0.775969 & 405.6761 & 0.776229 & 418.6252 & 0.748766 & 332.8745 \\
\hline 0.826531 & 414.5491 & 0.82859 & 431.7307 & 0.798081 & 336.6064 \\
\hline 0.873825 & 424.3697 & 0.877404 & 443.811 & 0.848765 & 341.5308 \\
\hline 0.924445 & 432.0753 & 0.927131 & 456.0046 & 0.901837 & 347.1574 \\
\hline 0.972871 & 443.316 & 0.978143 & 472.1187 & 0.95054 & 353.283 \\
\hline 0.925051 & 430.6322 & 0.919339 & 452.8603 & 0.9268 & 351.7759 \\
\hline 0.870073 & 420.4393 & 0.868549 & 439.4963 & 0.862437 & 347.1733 \\
\hline 0.817318 & 408.7946 & 0.819599 & 426.6182 & 0.824697 & 347.6305 \\
\hline 0.768956 & 399.9407 & 0.766668 & 412.9311 & 0.765591 & 343.4467 \\
\hline 0.718726 & 390.8578 & 0.7285 & 408.7925 & 0.726555 & 341.0682 \\
\hline 0.667154 & 382.1121 & 0.66899 & 399.7185 & 0.666335 & 337.7727 \\
\hline 0.618158 & 374.1427 & 0.61765 & 390.9779 & 0.628959 & 333.8852 \\
\hline 0.570597 & 367.2858 & 0.571219 & 381.5359 & 0.567625 & 331.1576 \\
\hline
\end{tabular}




\begin{tabular}{|l|l|l|l|l|l|}
\hline 0.520649 & 360.0216 & 0.523147 & 371.9851 & 0.528705 & 326.5955 \\
\hline 0.469906 & 353.2606 & 0.471722 & 361.7091 & 0.469322 & 319.1716 \\
\hline 0.421198 & 346.7237 & 0.419632 & 350.7703 & 0.428743 & 316.3339 \\
\hline 0.371647 & 338.4703 & 0.368706 & 340.7114 & 0.365232 & 307.2409 \\
\hline 0.318725 & 328.554 & 0.317516 & 330.8017 & 0.326735 & 302.0163 \\
\hline 0.269902 & 319.9749 & 0.267831 & 320.711 & 0.267192 & 293.5903 \\
\hline 0.218063 & 311.2129 & 0.216805 & 310.9397 & 0.228782 & 287.3873 \\
\hline 0.167898 & 302.6229 & 0.16899 & 300.4805 & 0.168431 & 277.3046 \\
\hline 0.121398 & 293.6887 & 0.12104 & 289.3611 & 0.117675 & 266.3263 \\
\hline 0.073628 & 280.5242 & 0.071605 & 274.1087 & 0.070129 & 250.6287 \\
\hline 0.027496 & 201.0551 & 0.029598 & 201.754 & 0.029972 & 170.2466 \\
\hline 0.019812 & 175.5842 & 0.01973 & 166.4008 & 0.018884 & 138.5911 \\
\hline
\end{tabular}

DMSO resolvated samples

Table S18. $\mathrm{N}_{2}$ isotherm data for DMSO resolvated UMCM-9 samples used.

\begin{tabular}{|l|l|l|l|l|l|}
\hline $\begin{array}{l}\text { DMSO } \\
\text { resolvation 1 }\end{array}$ & $\begin{array}{l}\text { DMSO } \\
\text { resolvation 2 }\end{array}$ & $\begin{array}{l}\text { DMSO } \\
\text { resolvation 3 }\end{array}$ & \\
\hline $\mathrm{P}_{0} \mathrm{P}_{0}$ & $\begin{array}{l}\mathrm{N}_{2} \text { uptake } \\
\left(\mathrm{cc} \mathrm{g}^{-1}\right)\end{array}$ & $\mathrm{P}_{0}$ & $\begin{array}{l}\mathrm{N}_{2} \text { uptake } \\
\left(\mathrm{cc} \mathrm{g}^{-1}\right)\end{array}$ & $\mathrm{P} \mathrm{P}_{0}$ & $\begin{array}{l}\mathrm{N}_{2} \text { uptake } \\
\left(\mathrm{cc}^{-1}\right)\end{array}$ \\
\hline 0.002915 & 45.5737 & 0.003072 & 33.9917 & 0.003895 & 26.9798 \\
\hline 0.003701 & 49.6327 & 0.003866 & 37.3792 & 0.004892 & 29.8461 \\
\hline 0.004531 & 53.4695 & 0.004783 & 40.7255 & 0.005948 & 32.7158 \\
\hline 0.00544 & 57.4738 & 0.005751 & 44.0725 & 0.00704 & 35.4089 \\
\hline 0.006472 & 61.3567 & 0.006741 & 47.3499 & 0.008117 & 37.9817 \\
\hline 0.007503 & 65.1279 & 0.00779 & 50.6556 & 0.00926 & 40.4996 \\
\hline 0.008586 & 68.6403 & 0.008831 & 53.6604 & 0.010367 & 42.7822 \\
\hline 0.009718 & 72.0494 & 0.009915 & 56.5289 & 0.011437 & 44.8551 \\
\hline 0.010945 & 75.3692 & 0.010986 & 59.228 & 0.012471 & 46.7384 \\
\hline 0.014228 & 82.8551 & 0.01373 & 65.0131 & 0.015528 & 51.5374 \\
\hline 0.018326 & 91.1964 & 0.017312 & 71.7835 & 0.019423 & 57.1504 \\
\hline 0.022395 & 99.7505 & 0.021585 & 79.5165 & 0.023471 & 62.9599 \\
\hline 0.02632 & 108.8474 & 0.02532 & 87.0857 & 0.027591 & 69.486 \\
\hline 0.030512 & 119.1824 & 0.031559 & 100.4469 & 0.031887 & 76.9591 \\
\hline 0.036933 & 134.6579 & 0.035097 & 108.5044 & 0.036626 & 85.4342 \\
\hline 0.041832 & 144.4291 & 0.041664 & 120.5084 & 0.041365 & 93.6933 \\
\hline 0.047308 & 151.9148 & 0.046257 & 126.6811 & 0.046243 & 100.6428 \\
\hline 0.053296 & 157.0684 & 0.05262 & 131.7746 & 0.052271 & 106.7858 \\
\hline 0.060951 & 161.1894 & 0.058379 & 134.8208 & 0.058735 & 110.6178 \\
\hline 0.068563 & 164.2671 & 0.064669 & 137.0248 & 0.064756 & 113.0329 \\
\hline 0.073873 & 166.183 & 0.070871 & 138.8776 & 0.071679 & 115.1636 \\
\hline 0.07927 & 167.8858 & 0.078064 & 140.5953 & 0.078472 & 116.9968 \\
\hline 0.085937 & 169.7558 & 0.084084 & 142.0383 & 0.084572 & 118.2104 \\
\hline 0.091759 & 171.3303 & 0.090229 & 143.0935 & 0.08921 & 119.4542 \\
\hline & & & & & \\
\hline
\end{tabular}




\begin{tabular}{|c|c|c|c|c|c|}
\hline 0.097084 & 172.6952 & 0.09558 & 144.2495 & 0.09424 & 120.6878 \\
\hline 0.104948 & 174.6029 & 0.10053 & 145.0547 & 0.100515 & 121.7984 \\
\hline 0.110193 & 176.0178 & 0.103988 & 145.9505 & 0.1046 & 122.5509 \\
\hline 0.164947 & 187.3255 & 0.148658 & 150.9241 & 0.148666 & 128.9885 \\
\hline 0.218893 & 198.9951 & 0.196918 & 155.7864 & 0.196743 & 135.4845 \\
\hline 0.268979 & 209.97 & 0.245548 & 160.2726 & 0.246793 & 140.9586 \\
\hline 0.318509 & 219.7628 & 0.298307 & 164.1656 & 0.299493 & 147.4995 \\
\hline 0.370608 & 230.5688 & 0.349485 & 168.6874 & 0.349485 & 152.6462 \\
\hline 0.420636 & 240.9561 & 0.398174 & 172.0485 & 0.400249 & 158.8889 \\
\hline 0.471162 & 251.1471 & 0.447897 & 175.272 & 0.449171 & 163.7635 \\
\hline 0.523715 & 261.863 & 0.495589 & 179.8828 & 0.497008 & 168.3879 \\
\hline 0.574529 & 271.9518 & 0.546483 & 183.396 & 0.545639 & 172.7038 \\
\hline 0.626563 & 282.7978 & 0.599147 & 186.6741 & 0.597414 & 178.3247 \\
\hline 0.67828 & 294.1155 & 0.648848 & 192.1257 & 0.64622 & 184.0473 \\
\hline 0.728878 & 304.8744 & 0.698447 & 195.2846 & 0.698978 & 189.0152 \\
\hline 0.779844 & 317.675 & 0.751009 & 198.5936 & 0.750696 & 193.4206 \\
\hline 0.830759 & 328.9391 & 0.800098 & 205.0492 & 0.800455 & 199.2649 \\
\hline 0.880484 & 339.0574 & 0.849544 & 208.6855 & 0.848146 & 204.7916 \\
\hline 0.929603 & 349.6408 & 0.897585 & 212.3324 & 0.900592 & 210.5757 \\
\hline 0.977676 & 365.6869 & 0.946572 & 215.4484 & 0.947315 & 214.4152 \\
\hline 0.920296 & 343.8183 & 0.924987 & 218.4416 & 0.926858 & 211.6868 \\
\hline 0.86871 & 330.3495 & 0.863682 & 213.8614 & 0.87897 & 215.9443 \\
\hline 0.819165 & 319.0846 & 0.828519 & 212.7282 & 0.815248 & 210.2278 \\
\hline 0.769988 & 308.7444 & 0.777785 & 213.2376 & 0.774793 & 206.8161 \\
\hline 0.718229 & 295.3847 & 0.717499 & 209.5869 & 0.729088 & 206.3237 \\
\hline 0.668576 & 286.1585 & 0.671416 & 209.8096 & 0.667012 & 203.6007 \\
\hline 0.618079 & 276.7387 & 0.616386 & 205.842 & 0.626425 & 202.2337 \\
\hline 0.571918 & 268.389 & 0.566212 & 202.089 & 0.567392 & 194.8206 \\
\hline 0.523268 & 260.5709 & 0.528742 & 201.8258 & 0.527752 & 191.006 \\
\hline 0.468442 & 251.2205 & 0.470167 & 196.1332 & 0.467677 & 187.4231 \\
\hline 0.420607 & 242.5379 & 0.429813 & 192.6361 & 0.415399 & 180.588 \\
\hline 0.369121 & 232.1056 & 0.367402 & 188.4928 & 0.366135 & 173.9126 \\
\hline 0.316489 & 219.9827 & 0.317104 & 183.7109 & 0.316077 & 167.3653 \\
\hline 0.268625 & 210.8223 & 0.266391 & 178.1732 & 0.266056 & 161.0449 \\
\hline 0.216303 & 200.2458 & 0.218117 & 172.6197 & 0.216472 & 153.879 \\
\hline 0.167508 & 190.3915 & 0.171911 & 166.4982 & 0.170302 & 146.0339 \\
\hline 0.118129 & 181.1118 & 0.117114 & 158.1271 & 0.116539 & 135.8172 \\
\hline 0.069681 & 170.2035 & 0.068163 & 147.8646 & 0.06742 & 123.5587 \\
\hline 0.027741 & 122.2911 & 0.026652 & 99.47 & 0.0231 & 71.3708 \\
\hline 0.018246 & 101.7412 & 0.018622 & 83.0035 & 0.019773 & 65.4987 \\
\hline
\end{tabular}




\section{$\mathrm{CH}_{2} \mathrm{Cl}_{2}$ RSE samples}

Table S19. $\mathrm{N}_{2}$ isotherm data for $\mathrm{CH}_{2} \mathrm{Cl}_{2}$ RSE UMCM-9 samples used.

\begin{tabular}{|c|c|c|c|c|c|}
\hline $\begin{array}{l}\mathrm{CH}_{2} \mathrm{Cl}_{2} \mathrm{RSE} \\
1\end{array}$ & & $\begin{array}{l}\mathrm{CH}_{2} \mathrm{Cl}_{2} \mathrm{RSE} \\
2\end{array}$ & & $\begin{array}{l}\mathrm{CH}_{2} \mathrm{Cl}_{2} \mathrm{RSE} \\
3\end{array}$ & \\
\hline $\mathrm{P} / \mathrm{P}_{0}$ & $\begin{array}{l}\mathrm{N}_{2} \text { uptake } \\
\left(\mathrm{cc} \mathrm{g}^{-1}\right)\end{array}$ & $\mathrm{P} / \mathrm{P}_{0}$ & $\begin{array}{l}\mathrm{N}_{2} \text { uptake } \\
\left(\mathrm{cc} \mathrm{g}^{-1}\right)\end{array}$ & $\mathrm{P} / \mathrm{P}_{0}$ & $\begin{array}{l}\mathrm{N}_{2} \text { uptake } \\
\left(\mathrm{cc} \mathrm{g}^{-1}\right)\end{array}$ \\
\hline 0.001447 & 116.4523 & 0.001588 & 130.2948 & 0.000972 & 108.0439 \\
\hline 0.002178 & 134.982 & 0.002066 & 144.8337 & 0.001772 & 133.5725 \\
\hline 0.002395 & 138.9366 & 0.002283 & 151.476 & 0.002103 & 143.3374 \\
\hline 0.002591 & 143.2571 & 0.00253 & 158.7704 & 0.002248 & 147.8403 \\
\hline 0.002786 & 148.1131 & 0.002827 & 166.8092 & 0.002435 & 152.7775 \\
\hline 0.003025 & 153.3894 & 0.003139 & 175.2291 & 0.002637 & 157.9219 \\
\hline 0.003271 & 159.2954 & 0.003472 & 184.0833 & 0.00286 & 163.7222 \\
\hline 0.00351 & 165.4601 & 0.004306 & 205.1881 & 0.00309 & 169.8648 \\
\hline 0.004147 & 180.9345 & 0.005147 & 226.4746 & 0.00402 & 193.9257 \\
\hline 0.005239 & 208.3784 & 0.010591 & 340.5368 & 0.005403 & 227.8658 \\
\hline 0.011087 & 331.8874 & 0.016832 & 429.893 & 0.010633 & 334.1795 \\
\hline 0.016007 & 405.6018 & 0.020761 & 479.2364 & 0.015747 & 407.3836 \\
\hline 0.02108 & 469.1804 & 0.025132 & 537.6119 & 0.020934 & 471.0472 \\
\hline 0.026037 & 533.6008 & 0.030272 & 615.476 & 0.025364 & 529.1401 \\
\hline 0.030314 & 598.3585 & 0.03639 & 727.1491 & 0.031177 & 621.1502 \\
\hline 0.03559 & 693.0795 & 0.040159 & 799.5146 & 0.035867 & 706.1994 \\
\hline 0.04038 & 788.479 & 0.046053 & 899.4316 & 0.040391 & 788.9645 \\
\hline 0.045091 & 878.0828 & 0.051417 & 961.2317 & 0.045498 & 871.1676 \\
\hline 0.050902 & 965.6228 & 0.05539 & 992.0528 & 0.051628 & 941.2669 \\
\hline 0.056424 & 1019.6191 & 0.061029 & 1017.3038 & 0.055129 & 966.1048 \\
\hline 0.06018 & 1040.1055 & 0.065255 & 1029.1007 & 0.060777 & 989.9886 \\
\hline 0.067691 & 1064.2009 & 0.072316 & 1044.3492 & 0.065294 & 1002.6435 \\
\hline 0.0711 & 1071.9806 & 0.077318 & 1052.8585 & 0.071093 & 1014.6445 \\
\hline 0.07516 & 1079.4438 & 0.082247 & 1060.0554 & 0.075869 & 1022.4883 \\
\hline 0.081644 & 1089.2881 & 0.089184 & 1068.701 & 0.081329 & 1030.1948 \\
\hline 0.086731 & 1095.9117 & 0.097274 & 1077.4867 & 0.086976 & 1037.089 \\
\hline 0.092224 & 1102.3259 & 0.104074 & 1084.1897 & 0.092509 & 1043.0523 \\
\hline 0.096754 & 1106.995 & 0.150047 & 1118.4231 & 0.09928 & 1049.6664 \\
\hline 0.146759 & 1142.2896 & 0.202566 & 1147.1212 & 0.147047 & 1085.1042 \\
\hline 0.199391 & 1166.0869 & 0.25581 & 1172.2727 & 0.196594 & 1111.3895 \\
\hline 0.246943 & 1181.8256 & 0.307749 & 1193.6108 & 0.246212 & 1132.5595 \\
\hline 0.297585 & 1196.0929 & 0.359326 & 1212.5533 & 0.299037 & 1152.9087 \\
\hline 0.347843 & 1209.0797 & 0.412171 & 1231.3989 & 0.3531 & 1171.6065 \\
\hline 0.401278 & 1221.7197 & 0.46698 & 1249.5584 & 0.406796 & 1188.347 \\
\hline 0.445783 & 1230.7123 & 0.520021 & 1266.5393 & 0.45962 & 1204.6405 \\
\hline 0.501469 & 1241.3553 & 0.56864 & 1285.4033 & 0.510203 & 1219.9316 \\
\hline 0.550751 & 1249.6068 & 0.620275 & 1302.5096 & 0.564094 & 1234.5798 \\
\hline
\end{tabular}




\begin{tabular}{|l|l|l|l|l|l|}
\hline 0.599381 & 1258.0658 & 0.672555 & 1318.4553 & 0.612942 & 1248.2764 \\
\hline 0.646238 & 1266.776 & 0.721913 & 1334.2355 & 0.66524 & 1262.8638 \\
\hline 0.707807 & 1277.5717 & 0.770924 & 1350.9305 & 0.71488 & 1275.847 \\
\hline 0.750214 & 1285.1656 & 0.822188 & 1368.5023 & 0.765219 & 1288.6657 \\
\hline 0.802708 & 1296.0333 & 0.87059 & 1385.0077 & 0.81684 & 1301.8901 \\
\hline 0.855051 & 1305.6711 & 0.921877 & 1400.3138 & 0.867114 & 1314.9498 \\
\hline 0.895677 & 1312.4655 & 0.973011 & 1417.6119 & 0.918641 & 1327.9665 \\
\hline 0.953136 & 1320.6001 & 0.91997 & 1397.8789 & 0.969456 & 1339.1775 \\
\hline 0.926925 & 1319.7297 & 0.86822 & 1380.9257 & 0.923072 & 1326.1903 \\
\hline 0.869025 & 1307.6254 & 0.819202 & 1363.2927 & 0.872884 & 1309.9276 \\
\hline 0.823506 & 1298.8751 & 0.76951 & 1345.3165 & 0.820132 & 1292.9453 \\
\hline 0.767386 & 1292.5609 & 0.718136 & 1326.8456 & 0.770211 & 1277.4286 \\
\hline 0.72634 & 1290.1339 & 0.667705 & 1309.1104 & 0.721313 & 1263.3955 \\
\hline 0.669641 & 1280.64 & 0.619209 & 1293.3367 & & \\
\hline 0.618536 & 1272.7917 & 0.57133 & 1277.0107 & & \\
\hline 0.568415 & 1265.6826 & 0.522392 & 1257.563 & & \\
\hline 0.517325 & 1258.5258 & 0.469278 & 1239.6799 & & \\
\hline 0.473869 & 1248.9706 & 0.419166 & 1223.2373 & & \\
\hline 0.422814 & 1238.9174 & 0.372511 & 1207.4994 & & \\
\hline 0.368721 & 1227.4534 & 0.322015 & 1189.2333 & & \\
\hline 0.322153 & 1215.8574 & 0.268648 & 1169.3812 & & \\
\hline 0.274312 & 1202.0764 & 0.221145 & 1149.0713 & & \\
\hline 0.225211 & 1186.4379 & 0.174425 & 1126.7898 & & \\
\hline 0.179526 & 1166.9921 & 0.129068 & 1100.1506 & & \\
\hline 0.1218 & 1133.1608 & 0.075361 & 1046.9547 & & \\
\hline 0.078344 & 1087.9042 & 0.029482 & 610.8469 & & \\
\hline & & 0.019014 & 457.6094 & & \\
\hline
\end{tabular}

\section{THF RSE samples}

Table S20. $\mathrm{N}_{2}$ isotherm data for THF RSE UMCM-9 samples used.

\begin{tabular}{|l|l|l|l|l|l|}
\hline THF RSE 1 & $\begin{array}{l}\mathrm{N}_{2} \text { uptake } \\
\left(\mathrm{cc} \mathrm{g}^{-1}\right)\end{array}$ & $\mathrm{P} / \mathrm{P}_{0}$ & $\begin{array}{l}\mathrm{N}_{2} \text { uptake } \\
\left(\mathrm{cc} \mathrm{g}^{-1}\right)\end{array}$ & $\mathrm{P} / \mathrm{P}_{0}$ & $\begin{array}{l}\mathrm{N}_{2} \text { uptake } \\
\left(\mathrm{cc} \mathrm{g}^{-1}\right)\end{array}$ \\
\hline 0.000832 & 99.5942 & 0.001508 & 126.247 & 0.001225 & 115.5137 \\
\hline 0.002301 & 142.05 & 0.002233 & 147.1904 & 0.001628 & 128.298 \\
\hline 0.002504 & 146.4827 & 0.002428 & 153.3263 & 0.00219 & 143.9659 \\
\hline 0.002735 & 151.5184 & 0.00266 & 159.6984 & 0.00232 & 147.8017 \\
\hline 0.002974 & 156.9191 & 0.002921 & 166.819 & 0.002471 & 152.045 \\
\hline 0.003213 & 162.8312 & 0.003197 & 174.3096 & 0.002658 & 156.4689 \\
\hline 0.003481 & 169.4519 & 0.003509 & 182.1718 & 0.002838 & 161.506 \\
\hline 0.00377 & 176.3008 & 0.004284 & 201.4355 & 0.00304 & 166.9016 \\
\hline 0.004067 & 183.4526 & 0.005024 & 219.6065 & 0.004214 & 196.9355 \\
\hline 0.005326 & 214.096 & 0.011569 & 351.5347 & 0.005079 & 218.2344 \\
\hline
\end{tabular}




\begin{tabular}{|c|c|c|c|c|c|}
\hline 0.011817 & 342.8071 & 0.015187 & 402.611 & 0.010957 & 337.6479 \\
\hline 0.01524 & 391.7376 & 0.021515 & 481.4713 & 0.015387 & 400.515 \\
\hline 0.02184 & 472.7428 & 0.025328 & 532.3002 & 0.02053 & 463.5185 \\
\hline 0.025408 & 518.2547 & 0.030482 & 609.8063 & 0.026308 & 540.0511 \\
\hline 0.030416 & 591.0793 & 0.035107 & 692.3314 & 0.030608 & 607.8764 \\
\hline 0.036096 & 691.2939 & 0.04124 & 804.4949 & 0.035651 & 698.0826 \\
\hline 0.04( & 763.7047 & 0.045437 & 875.5155 & 0.041061 & 797.1176 \\
\hline 0.046271 & 866.5284 & 0.05 & 942.608 & 0.045419 & 867.0708 \\
\hline 0.05185 & 35.0442 & 0.05 & 976.9294 & 0.050749 & 929.6936 \\
\hline 0.05 & 967.6193 & 0.06 & 1004.3724 & & 968.9416 \\
\hline 0.06 & 990.4816 & & 1015.0627 & & \\
\hline 0.06 & 1004.0999 & 0.0 & 102 & & 1297 \\
\hline 0.07 & 1013.3777 & 0.0 & 103 & & 0.7242 \\
\hline 0.07 & 1022.0157 & & & & 3.1973 \\
\hline 0.08 & 663 & & 105 & & 2689 \\
\hline 0.08 & & & 106 & & \\
\hline 0.09 & 742 & & 106 & & \\
\hline 0.09 & 431 & & 109 & & 316 \\
\hline 0.14 & 8901 & & 112 & & 292 \\
\hline 0.20 & 1105.5728 & 0.25 & 114 & & 534 \\
\hline 0.24 & 4027 & 0.30 & 116 & & 3337 \\
\hline 0.29 & 1136.2158 & 0.36 & 7816 & 525 & 7713 \\
\hline 0.35 & 1148.9348 & 0.41 & 5353 & 831 & 0488 \\
\hline 0.39 & 1160.2825 & 0.46 & 4054 & & .9423 \\
\hline 0.44 & 1172.6844 & 0.51 & 1229.0845 & 745 & .0121 \\
\hline 0.50 & 82.1654 & 0.56 & 1242.7775 & 951 & .3883 \\
\hline 0.550208 & 1192.3834 & 0.621978 & 1257.4718 & 0.560276 & 1212.3104 \\
\hline 0.596218 & 99.5045 & 0.67 & 1271.2526 & 518 & 1224.9671 \\
\hline 0.650963 & 1210.6653 & 0.723377 & 1284.4856 & 976 & 1236.9481 \\
\hline 0.69 & 219.92 & 0.77 & 3412 & 015 & 1247.757 \\
\hline 0.74 & 1232.2783 & 0.826915 & 703 & 064 & .3813 \\
\hline 0.80 & 1241.449 & 0.87 & 6743 & 649 & 1270.2222 \\
\hline 0.846273 & 1250.1346 & 0.925581 & 1345.275 & 0.86 & 1280.0681 \\
\hline 0.897566 & 1260.4004 & 0.974164 & 1360.0936 & 0.920875 & 1290.441 \\
\hline 0.949207 & 1271.8713 & 0.922341 & 1342.5945 & 0.970284 & 1302.6122 \\
\hline 0.92699 & 1268.9264 & 0.86906 & 1325.3928 & 0.92323 & 1287.8065 \\
\hline 0.869018 & 1253.6173 & 0.820528 & 1309.954 & 0.873288 & 1274.6669 \\
\hline 0.814902 & 1244.4093 & 0.769358 & 1292.3944 & 0.822048 & 1261.6045 \\
\hline 0.778487 & 1242.1168 & 0.719622 & 1277.4959 & 0.77217 & 1248.8852 \\
\hline 0.718213 & 1233.9232 & 0.669532 & 1263.4927 & 0.721911 & 1235.7479 \\
\hline 0.668 & 1033 & 0.61 & 251 & & \\
\hline 0.628696 & 19.4821 & 0.571612 & 1235.0824 & & \\
\hline 0.571259 & 1211.4343 & 0.523436 & 1221.8756 & & \\
\hline
\end{tabular}




\begin{tabular}{|l|l|l|l|l|l|}
\hline 0.52111 & 1202.3354 & 0.472091 & 1207.5101 & & \\
\hline 0.46902 & 1190.5912 & 0.419992 & 1192.3002 & & \\
\hline 0.419797 & 1181.14 & 0.372352 & 1177.8616 & & \\
\hline 0.368344 & 1168.615 & 0.319818 & 1161.6589 & & \\
\hline 0.321617 & 1156.5123 & 0.269373 & 1144.9363 & & \\
\hline 0.272785 & 1142.8503 & 0.22108 & 1127.7397 & & \\
\hline 0.224314 & 1125.7228 & 0.174172 & 1107.4753 & & \\
\hline 0.178781 & 1105.8773 & 0.129148 & 1082.9753 & & \\
\hline 0.129224 & 1078.1875 & 0.075397 & 1032.7118 & & \\
\hline 0.076839 & 1027.8474 & 0.029453 & 600.4489 & & \\
\hline & & 0.019841 & 462.0487 & & \\
\hline
\end{tabular}

\section{DMF RSE samples}

Table S21. $\mathrm{N}_{2}$ isotherm data for DMF RSE UMCM- 9 samples used.

\begin{tabular}{|l|l|l|l|l|l|}
\hline DMF RSE 1 & & DMF RSE 2 & & DMF RSE 3 & \\
\hline $\mathrm{P}^{\mathrm{P}_{0}}$ & $\begin{array}{l}\mathrm{N}_{2} \text { uptake } \\
\left(\mathrm{cc} \mathrm{g}^{-1}\right)\end{array}$ & $\mathrm{P} / \mathrm{P}_{0}$ & $\begin{array}{l}\mathrm{N}_{2} \text { uptake } \\
\left(\mathrm{cc} \mathrm{g}^{-1}\right)\end{array}$ & $\mathrm{P} / \mathrm{P}_{0}$ & $\begin{array}{l}\mathrm{N}_{2} \text { uptake } \\
\left(\mathrm{cc} \mathrm{g}^{-1}\right)\end{array}$ \\
\hline 0.000753 & 106.6805 & 0.00166 & 139.8508 & 0.001253 & 134.8667 \\
\hline 0.00212 & 151.286 & 0.002269 & 157.4192 & 0.001693 & 149.7097 \\
\hline 0.002258 & 154.2108 & 0.002457 & 162.5536 & 0.002132 & 162.597 \\
\hline 0.002417 & 157.6062 & 0.002653 & 167.9156 & 0.002298 & 167.0458 \\
\hline 0.002576 & 161.2743 & 0.002878 & 173.9339 & 0.002485 & 171.9515 \\
\hline 0.00275 & 165.2883 & 0.003139 & 180.2535 & 0.002709 & 177.0466 \\
\hline 0.002924 & 169.8004 & 0.0034 & 186.9513 & 0.002925 & 182.841 \\
\hline 0.003126 & 174.4722 & 0.004067 & 202.6221 & 0.003184 & 189.0045 \\
\hline 0.004284 & 203.841 & 0.005154 & 227.2196 & 0.004193 & 212.9794 \\
\hline 0.005131 & 223.9892 & 0.011221 & 338.4932 & 0.005165 & 234.578 \\
\hline 0.010884 & 340.0609 & 0.016202 & 401.4953 & 0.011266 & 341.3101 \\
\hline 0.015269 & 402.5985 & 0.021327 & 459.5968 & 0.016741 & 407.0125 \\
\hline 0.020523 & 464.6953 & 0.026118 & 517.9801 & 0.020264 & 444.8359 \\
\hline 0.025531 & 525.8939 & 0.030337 & 576.1373 & 0.026099 & 512.7076 \\
\hline 0.030777 & 602.4045 & 0.035991 & 666.0779 & 0.030399 & 571.3599 \\
\hline 0.035271 & 678.0693 & 0.041116 & 748.6905 & 0.036227 & 660.6899 \\
\hline 0.040771 & 776.6685 & 0.045444 & 808.1713 & 0.040081 & 718.726 \\
\hline 0.045157 & 846.0088 & 0.051272 & 866.5035 & 0.045131 & 783.0338 \\
\hline 0.051633 & 922.8174 & 0.055375 & 893.0149 & 0.050894 & 836.2141 \\
\hline 0.05549 & 952.1767 & 0.060616 & 912.3021 & 0.055259 & 860.7835 \\
\hline 0.060346 & 973.3549 & 0.066502 & 926.3175 & 0.06167 & 881.3915 \\
\hline 0.066939 & 990.7773 & 0.070779 & 933.8923 & 0.065351 & 889.5507 \\
\hline 0.071375 & 998.9802 & 0.075825 & 941.5056 & 0.07128 & 899.6696 \\
\hline 0.075377 & 1005.0114 & 0.08158 & 948.6484 & 0.076683 & 907.1129 \\
\hline 0.081246 & 1012.9382 & 0.088235 & 955.6933 & 0.081545 & 912.963 \\
\hline 0.086058 & 1018.3647 & 0.096216 & 962.9786 & 0.087978 & 919.6337 \\
\hline
\end{tabular}




\begin{tabular}{|l|l|l|l|l|l|}
\hline 0.090885 & 1023.1976 & 0.102479 & 968.1114 & 0.095232 & 926.3111 \\
\hline 0.097629 & 1029.3949 & 0.146278 & 995.1508 & 0.100426 & 930.6285 \\
\hline 0.155327 & 1062.9012 & 0.198927 & 1018.3929 & 0.146781 & 961.3499 \\
\hline 0.199789 & 1079.5764 & 0.252802 & 1037.2521 & 0.19645 & 985.7715 \\
\hline 0.24564 & 1092.5482 & 0.305277 & 1053.1692 & 0.247689 & 1006.6193 \\
\hline 0.297975 & 1105.3011 & 0.358485 & 1067.7812 & 0.299094 & 1025.1682 \\
\hline 0.348697 & 1115.6143 & 0.411091 & 1080.6901 & 0.353496 & 1043.244 \\
\hline 0.401293 & 1125.5665 & 0.464219 & 1093.4642 & 0.405794 & 1059.205 \\
\hline 0.446008 & 1131.8226 & 0.517158 & 1105.1432 & 0.45766 & 1075.1533 \\
\hline 0.501491 & 1139.6519 & 0.570112 & 1117.3403 & 0.511997 & 1090.5348 \\
\hline 0.548486 & 1146.5498 & 0.620318 & 1128.4684 & 0.563791 & 1105.8026 \\
\hline 0.598845 & 1153.7507 & 0.674128 & 1139.7833 & 0.613619 & 1119.692 \\
\hline 0.648163 & 1160.6776 & 0.726219 & 1150.7067 & 0.666025 & 1133.9014 \\
\hline 0.703132 & 1168.9252 & 0.776708 & 1161.7095 & 0.718597 & 1147.5649 \\
\hline 0.747044 & 1174.2314 & 0.828104 & 1172.8937 & 0.770946 & 1161.3206 \\
\hline 0.80226 & 1183.0555 & 0.879543 & 1183.9877 & 0.820838 & 1174.1542 \\
\hline 0.847937 & 1188.7726 & 0.928596 & 1194.1884 & 0.869801 & 1187.0037 \\
\hline 0.897508 & 1196.176 & 0.977332 & 1203.5571 & 0.919333 & 1200.2642 \\
\hline 0.957427 & 1202.4419 & 0.921579 & 1191.5598 & 0.969938 & 1213.5154 \\
\hline 0.925753 & 1197.2561 & 0.8698 & 1179.5453 & 0.922323 & 1197.0596 \\
\hline 0.868171 & 1188.0707 & 0.819999 & 1167.7549 & 0.873093 & 1180.0527 \\
\hline 0.826293 & 1184.6471 & 0.769698 & 1156.1365 & 0.822005 & 1163.321 \\
\hline 0.766033 & 1179.7196 & 0.720072 & 1144.1981 & 0.77302 & 1148.4971 \\
\hline 0.725327 & 1175.2516 & 0.669517 & 1132.8591 & 0.72052 & 1133.4366 \\
\hline 0.668353 & 1171.0487 & 0.622145 & 1122.2969 & & \\
\hline 0.620953 & 1165.5941 & 0.573048 & 1110.9485 & & \\
\hline 0.568748 & 1159.8817 & 0.52179 & 1098.5947 & & \\
\hline 0.519105 & 1151.9012 & 0.471047 & 1087.303 & & \\
\hline 0.471691 & 1146.1461 & 0.418912 & 1074.8542 & & \\
\hline 0.423133 & 1138.4492 & 0.370728 & 1063.1659 & & \\
\hline 0.370609 & 1130.0868 & 0.318978 & 1049.5388 & & \\
\hline 0.322413 & 1120.8896 & 0.270047 & 1036.326 & & \\
\hline 0.275658 & 1109.5636 & 0.221015 & 1021.2796 & & \\
\hline 0.227172 & 1095.7021 & 0.174483 & 1004.3187 & & \\
\hline 0.171805 & 1075.6411 & 0.129641 & 983.1228 & & \\
\hline 0.121742 & 1050.0608 & 0.076252 & 940.1655 & & \\
\hline & & 0.029851 & 575.9409 & & \\
\hline & & 0.019188 & 436.1349 & & \\
\hline & & & & & \\
\hline
\end{tabular}


DMSO RSE samples

Table S22. $\mathrm{N}_{2}$ isotherm data for DMSO RSE UMCM-9 samples used.

\begin{tabular}{|c|c|c|c|c|c|}
\hline DMSO RSE 1 & & DMSO RSE 2 & & DMSO RSE 3 & \\
\hline $\mathrm{P} / \mathrm{P}_{0}$ & $\begin{array}{l}\mathrm{N}_{2} \text { uptake } \\
\left(\mathrm{cc} \mathrm{g}^{-1}\right)\end{array}$ & $\mathrm{P} / \mathrm{P}_{0}$ & $\begin{array}{l}\mathrm{N}_{2} \text { uptake } \\
\left(\mathrm{cc} \mathrm{g}^{-1}\right)\end{array}$ & $\mathrm{P} / \mathrm{P}_{0}$ & $\begin{array}{l}\mathrm{N}_{2} \text { uptake } \\
\left(\mathrm{cc} \mathrm{g} \mathrm{g}^{-1}\right)\end{array}$ \\
\hline 0.001288 & 96.0082 & 0.00166 & 107.9172 & 0.001059 & 77.7508 \\
\hline 0.002164 & 114.0716 & 0.00224 & 120.2242 & 0.001563 & 89.4876 \\
\hline 0.002308 & 116.4553 & 0.002523 & 125.8568 & 0.002226 & 101.5963 \\
\hline 0.002475 & 119.1109 & 0.00282 & 131.6187 & 0.002392 & 104.5363 \\
\hline 0.002656 & 121.9861 & 0.003168 & 138.0065 & 0.002586 & 107.7763 \\
\hline 0.00283 & 125.1624 & 0.003545 & 144.658 & 0.002817 & 111.1439 \\
\hline 0.003032 & 128.6833 & 0.003951 & 151.5927 & 0.00304 & 114.9969 \\
\hline 0.003249 & 132.32 & 0.004386 & 158.8397 & 0.003292 & 119.0131 \\
\hline 0.004197 & 148.4951 & 0.005277 & 172.7401 & 0.004301 & 133.6643 \\
\hline 0.005167 & 164.3745 & 0.011627 & 252.2136 & 0.005331 & 147.7923 \\
\hline 0.010623 & 236.6508 & 0.01544 & 286.3076 & 0.011223 & 211.291 \\
\hline 0.016782 & 293.0219 & 0.020884 & 330.3972 & 0.016223 & 249.7377 \\
\hline 0.021008 & 326.5128 & 0.026249 & 377.508 & 0.021056 & 283.6839 \\
\hline 0.025328 & 362.7814 & 0.030866 & 424.2672 & 0.025681 & 318.728 \\
\hline 0.030821 & 416.0493 & 0.035303 & 471.5812 & 0.030075 & 355.7841 \\
\hline 0.035829 & 470.4579 & 0.040246 & 520.6962 & 0.036328 & 411.6847 \\
\hline 0.040569 & 520.2985 & 0.045676 & 564.2274 & 0.040585 & 446.8318 \\
\hline 0.045099 & 558.3901 & 0.050656 & 590.5933 & 0.046103 & 480.7905 \\
\hline 0.051112 & 592.6505 & 0.055622 & 606.33 & 0.050526 & 499.5286 \\
\hline 0.055404 & 607.6891 & 0.060239 & 615.5448 & 0.057096 & 515.2463 \\
\hline 0.061555 & 620.1567 & 0.066002 & 623.8464 & 0.060287 & 520.2543 \\
\hline 0.065021 & 625.1736 & 0.071787 & 630.4708 & 0.066835 & 527.8772 \\
\hline 0.0711 & 631.7344 & 0.078811 & 636.7833 & 0.071064 & 531.7761 \\
\hline 0.075927 & 636.1233 & 0.085538 & 641.8764 & 0.076711 & 536.1888 \\
\hline 0.080797 & 639.8948 & 0.091946 & 646.1163 & 0.081617 & 539.545 \\
\hline 0.086239 & 643.6499 & 0.098333 & 650.1694 & 0.088453 & 543.6298 \\
\hline 0.09218 & 647.2247 & 0.105292 & 653.9594 & 0.094274 & 546.7723 \\
\hline 0.098121 & 650.5894 & 0.156209 & 676.0826 & 0.100382 & 549.7729 \\
\hline 0.148069 & 669.5587 & 0.20809 & 693.1686 & 0.148387 & 568.453 \\
\hline 0.201164 & 682.6027 & 0.261276 & 708.1066 & 0.19668 & 582.8725 \\
\hline 0.24729 & 691.455 & 0.315034 & 721.64 & 0.248669 & 595.7963 \\
\hline 0.300573 & 699.8193 & 0.367336 & 734.1447 & 0.302084 & 607.6104 \\
\hline 0.346598 & 706.3364 & 0.418528 & 745.5418 & 0.354144 & 618.4812 \\
\hline 0.398564 & 713.4916 & 0.470924 & 756.6505 & 0.406111 & 628.4979 \\
\hline 0.450921 & 718.9785 & 0.522805 & 767.8853 & 0.459483 & 638.9483 \\
\hline 0.49966 & 724.5897 & 0.572685 & 778.1657 & 0.510852 & 648.3237 \\
\hline 0.545027 & 729.0024 & 0.625704 & 789.1121 & 0.562963 & 657.6418 \\
\hline 0.601407 & 734.6564 & 0.674903 & 799.1848 & 0.617019 & 667.5389 \\
\hline
\end{tabular}




\begin{tabular}{|l|l|l|l|l|l|}
\hline 0.646151 & 739.8237 & 0.728002 & 810.2742 & 0.6681 & 676.5011 \\
\hline 0.698458 & 745.2098 & 0.777817 & 821.0779 & 0.718626 & 684.8661 \\
\hline 0.748318 & 751.8749 & 0.829488 & 832.4011 & 0.768986 & 693.513 \\
\hline 0.803526 & 757.3835 & 0.881261 & 843.5163 & 0.821558 & 702.8256 \\
\hline 0.849688 & 761.1869 & 0.930894 & 853.7081 & 0.869253 & 711.964 \\
\hline 0.903167 & 766.769 & 0.978477 & 862.9576 & 0.919729 & 720.8604 \\
\hline 0.947781 & 770.9336 & 0.920246 & 849.9786 & 0.968246 & 730.0968 \\
\hline 0.929241 & 766.778 & 0.86964 & 838.4755 & 0.920442 & 718.1959 \\
\hline 0.86668 & 761.4622 & 0.820115 & 826.4901 & 0.871652 & 707.0812 \\
\hline 0.828406 & 758.142 & 0.769525 & 814.7048 & 0.821465 & 695.6078 \\
\hline 0.767032 & 754.7618 & 0.718202 & 802.5684 & 0.77199 & 685.6299 \\
\hline 0.729119 & 752.9872 & 0.667379 & 791.4996 & 0.721947 & 676.278 \\
\hline 0.66598 & 747.6656 & 0.618325 & 780.9702 & & \\
\hline 0.629065 & 744.766 & 0.570706 & 770.385 & & \\
\hline 0.573068 & 740.038 & 0.521986 & 759.848 & & \\
\hline 0.517455 & 734.8794 & 0.472642 & 748.8486 & & \\
\hline 0.472566 & 730.004 & 0.419739 & 737.8431 & & \\
\hline 0.421664 & 724.28 & 0.371112 & 727.0612 & & \\
\hline 0.367063 & 717.9954 & 0.31926 & 715.2783 & & \\
\hline 0.321422 & 711.4879 & 0.268017 & 703.355 & & \\
\hline 0.27374 & 703.878 & 0.218296 & 690.9037 & & \\
\hline 0.223322 & 694.3383 & 0.171134 & 677.3193 & & \\
\hline 0.177855 & 683.5868 & 0.125031 & 660.6889 & & \\
\hline 0.129803 & 668.6545 & 0.078971 & 635.1545 & & \\
\hline & & 0.02875 & 404.7621 & & \\
\hline & & 0.019282 & 318.8458 & & \\
\hline
\end{tabular}

IRMOF-3

Pristine samples

Table S23. $\mathrm{N}_{2}$ isotherm data for pristine IRMOF-3 samples used. Samples are labelled with their BET surface areas, given in parentheses.

\begin{tabular}{|l|l|l|l|l|l|}
\hline $\begin{array}{l}\text { Pristine } \\
(2846.728)\end{array}$ & $\begin{array}{l}\text { Pristine } \\
(2723.582)\end{array}$ & & $\begin{array}{l}\text { Pristine } \\
(2758.320)\end{array}$ & \\
\hline $\mathrm{P} / \mathrm{P}_{0}$ & $\mathrm{~N}_{2}$ uptake $\left(\mathrm{cc} \mathrm{g}^{-1}\right)$ & $\mathrm{P} / \mathrm{P}_{0}$ & $\begin{array}{l}\mathrm{N}_{2} \text { uptake } \\
\left.(\mathrm{cc} \mathrm{g})^{-1}\right)\end{array}$ & $\mathrm{P} \mathrm{P}_{0}$ & $\begin{array}{l}\mathrm{N}_{2} \text { uptake } \\
\left(\mathrm{cc} \mathrm{g}^{-1}\right)\end{array}$ \\
\hline 0.000856 & 186.6838 & 0.001553 & 301.3788 & 0.001525 & 315.5685 \\
\hline 0.0015 & 283.985 & 0.002041 & 381.5295 & 0.00205 & 402.8175 \\
\hline 0.002092 & 383.5995 & 0.002056 & 386.0941 & 0.002035 & 404.6269 \\
\hline 0.002027 & 386.4686 & 0.002092 & 391.1812 & 0.002057 & 406.6476 \\
\hline 0.002041 & 389.5788 & 0.002135 & 396.5203 & 0.002086 & 408.7935 \\
\hline 0.00207 & 393.0332 & 0.002207 & 402.6256 & 0.002107 & 411.2892 \\
\hline 0.0021 & 396.8442 & 0.003364 & 478.1078 & 0.003035 & 474.6723 \\
\hline 0.003256 & 493.3278 & 0.004205 & 506.9238 & 0.004236 & 518.9852 \\
\hline
\end{tabular}




\begin{tabular}{|c|c|c|c|c|c|}
\hline 0.00417 & 530.8211 & 0.005096 & 527.8423 & 0.005099 & 538.9257 \\
\hline 0.005077 & 554.2401 & 0.012578 & 595.5035 & 0.010141 & 593.5232 \\
\hline 0.012115 & 623.6217 & 0.015971 & 606.9885 & 0.017506 & 620.9821 \\
\hline 0.015912 & 637.1946 & 0.020614 & 617.7178 & 0.02021 & 626.9193 \\
\hline 0.021728 & 650.1838 & 0.026493 & 627.1177 & 0.025287 & 635.2256 \\
\hline 0.026345 & 657.2733 & 0.030734 & 632.5758 & 0.031185 & 642.3717 \\
\hline 0.031898 & 663.8951 & 0.036067 & 638.1304 & 0.036047 & 647.0881 \\
\hline 0.035651 & 667.5847 & 0.042098 & 643.4355 & 0.041398 & 651.3784 \\
\hline 0.040392 & 671.5142 & 0.047675 & 647.7037 & 0.045238 & 654.0895 \\
\hline 0.045249 & 674.9889 & 0.052951 & 651.3242 & 0.051122 & 657.7059 \\
\hline 0.051014 & 678.58 & 0.059319 & 655.1876 & 0.056127 & 660.4324 \\
\hline 0.05664 & 681.6474 & 0.064415 & 658.2393 & 0.060615 & 662.6427 \\
\hline 0.062888 & 684.6675 & 0.070323 & 661.3358 & 0.065844 & 664.9982 \\
\hline 0.068002 & 686.9179 & 0.075685 & 663.9828 & 0.071044 & 667.1672 \\
\hline 0.07234 & 688.7151 & 0.081392 & 666.6849 & 0.07651 & 669.2589 \\
\hline 0.077893 & 690.7998 & 0.087624 & 669.5057 & 0.082558 & 671.4055 \\
\hline 0.084507 & 693.0999 & 0.093827 & 672.1678 & 0.088909 & 673.461 \\
\hline 0.08954 & 694.7747 & 0.101028 & 675.0586 & 0.095166 & 675.3439 \\
\hline 0.096139 & 696.7136 & 0.106606 & 677.2594 & 0.101035 & 676.9879 \\
\hline 0.100156 & 697.9298 & 0.152348 & 691.6379 & 0.145007 & 686.9564 \\
\hline 0.146086 & 707.9977 & 0.201511 & 704.6813 & 0.195856 & 695.4618 \\
\hline 0.195886 & 716.0574 & 0.252679 & 717.1183 & 0.246467 & 702.7219 \\
\hline 0.249526 & 722.6945 & 0.30297 & 728.9453 & 0.29674 & 708.8449 \\
\hline 0.301324 & 728.2996 & 0.353607 & 740.7395 & 0.347581 & 714.4917 \\
\hline 0.348439 & 732.6804 & 0.40374 & 752.4312 & 0.399486 & 720.1123 \\
\hline 0.395319 & 737.1478 & 0.454405 & 763.8578 & 0.451133 & 725.0294 \\
\hline 0.450467 & 741.5918 & 0.505839 & 776.171 & 0.501909 & 730.0079 \\
\hline 0.49756 & 745.2385 & 0.55754 & 788.3908 & 0.552657 & 735.8967 \\
\hline 0.545129 & 748.7983 & 0.608729 & 800.7709 & 0.606188 & 741.2461 \\
\hline 0.602786 & 752.8481 & 0.659272 & 813.0172 & 0.658129 & 746.4482 \\
\hline 0.645226 & 755.8998 & 0.708378 & 824.7012 & 0.709502 & 751.314 \\
\hline 0.696753 & 759.4926 & 0.757699 & 836.9116 & 0.760494 & 756.589 \\
\hline 0.748455 & 762.9728 & 0.808285 & 849.5768 & 0.81048 & 761.8195 \\
\hline 0.798899 & 766.572 & 0.857182 & 862.1201 & 0.85853 & 767.2512 \\
\hline 0.846555 & 769.8096 & 0.907402 & 874.8718 & 0.909616 & 773.0703 \\
\hline 0.901308 & 772.6852 & 0.957571 & 886.5629 & 0.956933 & 779.3196 \\
\hline 0.945796 & 776.0291 & 0.923567 & 877.0425 & 0.920526 & 775.5751 \\
\hline 0.927419 & 775.5742 & 0.87605 & 864.5355 & 0.871857 & 771.0773 \\
\hline 0.867479 & 772.6751 & 0.825931 & 850.8779 & 0.822944 & 766.4503 \\
\hline 0.828558 & 771.0573 & 0.775761 & 837.6851 & 0.772333 & 761.6368 \\
\hline 0.766262 & 767.8973 & 0.725247 & 825.0805 & 0.722534 & 756.8789 \\
\hline 0.72658 & 765.6781 & 0.675531 & 813.2606 & 0.67201 & 752.2485 \\
\hline 0.669267 & 762.3526 & 0.625584 & 801.8733 & 0.623966 & 748.0349 \\
\hline
\end{tabular}




\begin{tabular}{|l|l|l|l|l|l|}
\hline 0.620096 & 760.2639 & 0.575293 & 790.8622 & 0.576599 & 743.7758 \\
\hline 0.57015 & 756.2864 & 0.528207 & 780.7929 & 0.52811 & 738.899 \\
\hline 0.516875 & 752.7338 & 0.477901 & 769.5461 & 0.477895 & 732.3249 \\
\hline 0.472993 & 749.2807 & 0.426654 & 755.666 & 0.428262 & 724.582 \\
\hline 0.420128 & 744.8826 & 0.376377 & 744.7269 & 0.375572 & 719.4034 \\
\hline 0.368792 & 740.3433 & 0.324361 & 733.1467 & 0.327673 & 714.1079 \\
\hline 0.321713 & 735.1476 & 0.275579 & 721.6042 & 0.276055 & 708.1308 \\
\hline 0.272689 & 729.8566 & 0.224051 & 709.2468 & 0.226019 & 701.4277 \\
\hline 0.222787 & 723.1833 & 0.175858 & 697.3744 & 0.171482 & 692.9409 \\
\hline 0.175225 & 715.6951 & 0.128722 & 684.0579 & 0.122662 & 683.3801 \\
\hline 0.12525 & 705.7328 & 0.071128 & 662.3439 & 0.075503 & 669.7415 \\
\hline 0.078142 & 692.0454 & 0.028707 & 630.6712 & 0.029524 & 641.2546 \\
\hline 0.026616 & 657.5483 & 0.017229 & 610.0887 & 0.019038 & 625.1136 \\
\hline
\end{tabular}

\section{$\mathrm{CH}_{2} \mathrm{Cl}_{2}$ resolvated samples}

Table S24. $\mathrm{N}_{2}$ isotherm data for $\mathrm{CH}_{2} \mathrm{Cl}_{2}$ resolvated IRMOF-3 samples used.

\begin{tabular}{|l|l|l|l|l|l|}
\hline $\begin{array}{l}\mathrm{CH}_{2} \mathrm{Cl}_{2} \\
\text { resolvation 1 }\end{array}$ & $\begin{array}{l}\mathrm{CH}_{2} \mathrm{Cl}_{2} \\
\text { resolvation 2 }\end{array}$ & & $\begin{array}{l}\mathrm{CH}_{2} \mathrm{Cl}_{2} \\
\text { resolvation 3 }\end{array}$ \\
\hline $\mathrm{P} / \mathrm{P}_{0}$ & $\mathrm{~N}_{2}$ uptake $\left(\mathrm{cc} \mathrm{g}^{-1}\right)$ & $\mathrm{P} / \mathrm{P}_{0}$ & $\begin{array}{l}\mathrm{N}_{2} \text { uptake } \\
\left(\mathrm{cc} \mathrm{g}^{-1}\right)\end{array}$ & $\mathrm{P} / \mathrm{P}_{0}$ & $\begin{array}{l}\mathrm{N}_{2} \text { uptake } \\
\left(\mathrm{cc} \mathrm{g}^{-1}\right)\end{array}$ \\
\hline 0.001569 & 338.4936 & 0.000792 & 175.8427 & 0.001555 & 275.9629 \\
\hline 0.002117 & 417.7809 & 0.001713 & 332.0263 & 0.002027 & 351.1375 \\
\hline 0.002117 & 422.1602 & 0.002166 & 391.2234 & 0.002056 & 356.9337 \\
\hline 0.002167 & 426.7111 & 0.002202 & 397.131 & 0.002115 & 362.9989 \\
\hline 0.002217 & 431.9087 & 0.002281 & 403.4106 & 0.002194 & 369.7578 \\
\hline 0.002304 & 437.4185 & 0.00236 & 410.313 & 0.002274 & 377.0309 \\
\hline 0.003276 & 488.6112 & 0.002461 & 417.7493 & 0.003328 & 437.121 \\
\hline 0.004305 & 520.0953 & 0.003023 & 449.28 & 0.004135 & 462.4313 \\
\hline 0.005277 & 539.7979 & 0.004325 & 492.6904 & 0.005341 & 487.238 \\
\hline 0.013722 & 603.152 & 0.005153 & 510.1216 & 0.010834 & 536.9028 \\
\hline 0.016199 & 610.6912 & 0.011047 & 564.5229 & 0.015354 & 553.4458 \\
\hline 0.020843 & 620.6934 & 0.016048 & 581.875 & 0.020847 & 565.4153 \\
\hline 0.025105 & 627.5081 & 0.020302 & 591.1249 & 0.025534 & 572.5602 \\
\hline 0.032031 & 635.788 & 0.025109 & 598.6034 & 0.031311 & 579.3698 \\
\hline 0.037258 & 640.8804 & 0.030996 & 605.5414 & 0.037458 & 585.0612 \\
\hline 0.043248 & 645.6309 & 0.037329 & 611.4366 & 0.043838 & 589.8942 \\
\hline 0.049029 & 649.5041 & 0.043806 & 616.3894 & 0.049891 & 593.8176 \\
\hline 0.054306 & 652.7418 & 0.049981 & 620.3121 & 0.055064 & 596.8377 \\
\hline 0.061095 & 656.3474 & 0.055407 & 623.3171 & 0.06196 & 600.4754 \\
\hline 0.066041 & 658.6459 & 0.062107 & 626.6713 & 0.067497 & 603.0772 \\
\hline 0.071556 & 661.032 & 0.067634 & 629.1838 & 0.073615 & 605.7578 \\
\hline 0.076725 & 663.0923 & 0.07324 & 631.5652 & 0.078375 & 607.725 \\
\hline 0.0817 & 665.0273 & 0.078342 & 633.5996 & 0.084515 & 610.1065 \\
\hline & & & & & \\
\hline
\end{tabular}




\begin{tabular}{|c|c|c|c|c|c|}
\hline 0.087352 & 667.0994 & 0.084639 & 635.9718 & 0.088911 & 611.7842 \\
\hline 0.09584 & 669.9767 & 0.089324 & 637.6635 & 0.095145 & 613.9935 \\
\hline 0.10255 & 672.0931 & 0.09601 & 639.9949 & 0.100261 & 615.7503 \\
\hline 0.108173 & 673.7773 & 0.100832 & 641.6157 & 0.105631 & 617.4175 \\
\hline 0.15777 & 686.3387 & 0.107503 & 643.666 & 0.157105 & 630.986 \\
\hline 0.210514 & 697.6058 & 0.158398 & 656.5054 & 0.210607 & 642.6088 \\
\hline 0.263459 & 707.0687 & 0.212142 & 667.5662 & 0.261958 & 652.3807 \\
\hline 0.31508 & 715.9657 & 0.263288 & 676.8102 & 0.313905 & 661.4153 \\
\hline 0.365347 & 724.3108 & 0.315363 & 685.3171 & 0.367763 & 670.4456 \\
\hline 0.418681 & 732.8501 & 0.365444 & 693.7751 & 0.420197 & 679.1678 \\
\hline 0.469315 & 740.9766 & 0.416879 & 701.5796 & 0.472057 & 687.9391 \\
\hline 0.520914 & 748.7346 & 0.469364 & 710.1993 & 0.520705 & 695.9149 \\
\hline 0.571037 & 756.1835 & 0.522144 & 718.138 & 0.571061 & 704.4914 \\
\hline 0.623918 & 764.6137 & 0.571066 & 727.221 & 0.621721 & 712.9328 \\
\hline 0.675236 & 772.4561 & 0.621666 & 735.9577 & 0.673378 & 721.7628 \\
\hline 0.72623 & 780.0464 & 0.671437 & 744.5289 & 0.722345 & 729.8644 \\
\hline 0.776375 & 788.05 & 0.721727 & 752.9969 & 0.773319 & 737.7946 \\
\hline 0.828154 & 796.8931 & 0.77219 & 762.02 & 0.824808 & 745.0756 \\
\hline 0.87616 & 805.7294 & 0.821206 & 770.8575 & 0.875156 & 752.392 \\
\hline 0.92665 & 814.9212 & 0.871914 & 780.487 & 0.926144 & 759.556 \\
\hline 0.976334 & 826.1446 & 0.921225 & 789.9267 & 0.976936 & 763.4883 \\
\hline 0.921236 & 813.9349 & 0.971097 & 799.5429 & 0.92331 & 758.5491 \\
\hline 0.868205 & 803.4054 & 0.923168 & 790.5648 & 0.872729 & 750.6476 \\
\hline 0.820292 & 793.2141 & 0.870244 & 780.0911 & 0.822802 & 742.0405 \\
\hline 0.767246 & 784.0593 & 0.819825 & 769.9959 & 0.771742 & 734.2088 \\
\hline 0.720276 & 775.7193 & 0.771629 & 761.5547 & 0.720449 & 725.9019 \\
\hline 0.669325 & 767.4773 & 0.720871 & 752.1813 & 0.669774 & 718.4359 \\
\hline 0.619533 & 759.191 & 0.670358 & 742.7482 & 0.620711 & 710.8016 \\
\hline 0.570864 & 751.0447 & 0.620672 & 733.6319 & 0.57271 & 703.6739 \\
\hline 0.521116 & 743.6111 & 0.570361 & 724.5036 & 0.523517 & 696.7141 \\
\hline 0.4692 & 735.5685 & 0.522698 & 715.8205 & 0.472529 & 688.4866 \\
\hline 0.419739 & 727.5466 & 0.473566 & 707.9447 & 0.422784 & 678.1921 \\
\hline 0.372374 & 720.1584 & 0.419311 & 697.8353 & 0.370735 & 670.4722 \\
\hline 0.317967 & 711.6029 & 0.369179 & 690.1627 & 0.320837 & 662.5975 \\
\hline 0.268319 & 703.5771 & 0.318594 & 681.6603 & 0.270642 & 654.1259 \\
\hline 0.218095 & 695.3698 & 0.270658 & 674.3046 & 0.219676 & 644.6062 \\
\hline 0.170362 & 686.2289 & 0.219 & 667.6457 & 0.17094 & 634.4542 \\
\hline 0.123709 & 675.9309 & 0.168494 & 658.8879 & 0.123339 & 622.7377 \\
\hline 0.075703 & 661.3364 & & & 0.074945 & \begin{tabular}{|l|}
606.726 \\
\end{tabular} \\
\hline 0.027243 & 629.6801 & & & 0.025003 & \begin{tabular}{|l}
572.0439 \\
\end{tabular} \\
\hline 0.01874 & 615.9101 & & & 0.017991 & \begin{tabular}{|l}
559.9057 \\
\end{tabular} \\
\hline
\end{tabular}




\section{THF resolvated samples}

Table S25. $\mathrm{N}_{2}$ isotherm data for THF resolvated IRMOF-3 samples used.

\begin{tabular}{|c|c|c|c|c|c|}
\hline $\begin{array}{l}\text { THF } \\
\text { resolvation } 1\end{array}$ & & $\begin{array}{l}\text { THF } \\
\text { resolvation } 2\end{array}$ & & $\begin{array}{l}\text { THF } \\
\text { resolvation } 3\end{array}$ & \\
\hline $\mathrm{P} / \mathrm{P}_{0}$ & $\mathrm{~N}_{2}$ uptake $\left(\mathrm{cc} \mathrm{g}^{-1}\right)$ & $\mathrm{P} / \mathrm{P}_{0}$ & $\begin{array}{l}\mathrm{N}_{2} \text { uptake } \\
\left(\mathrm{cc} \mathrm{g}^{-1}\right)\end{array}$ & $\mathrm{P} / \mathrm{P}_{0}$ & $\begin{array}{l}\mathrm{N}_{2} \text { uptake } \\
\left(\mathrm{cc} \mathrm{g}^{-1}\right)\end{array}$ \\
\hline 0.0015 & 278.2234 & 0.000936 & 202.5886 & 0.001533 & 274.8725 \\
\hline 0.002059 & 365.8123 & 0.00167 & 320.6308 & 0.002173 & 359.6135 \\
\hline 0.002074 & 369.548 & 0.002152 & 376.5596 & 0.002187 & 364.26 \\
\hline 0.00211 & 373.6219 & 0.002188 & 382.174 & 0.002253 & 369.0916 \\
\hline 0.002147 & 378.149 & 0.002274 & 388.4389 & 0.00234 & 374.4931 \\
\hline 0.002176 & 383.1598 & 0.002375 & 394.9733 & 0.00242 & 380.1977 \\
\hline 0.003118 & 463.8061 & 0.002476 & 402.4081 & 0.003074 & 414.2841 \\
\hline 0.004235 & 512.4102 & 0.003008 & 429.244 & 0.004076 & 447.0426 \\
\hline 0.005301 & 542.5121 & 0.004404 & 470.3538 & 0.005108 & 467.4171 \\
\hline 0.011831 & 605.4714 & 0.005124 & 483.6363 & 0.010384 & 515.1585 \\
\hline 0.016735 & 622.7297 & 0.010917 & 533.1635 & 0.016342 & 535.0582 \\
\hline 0.021191 & 632.52 & 0.016099 & 549.6676 & 0.020171 & 542.6353 \\
\hline 0.026632 & 641.0571 & 0.020496 & 558.3068 & 0.026864 & 551.9581 \\
\hline 0.03064 & 645.954 & 0.025397 & 565.2557 & 0.031841 & 557.1686 \\
\hline 0.035434 & 650.7918 & 0.031406 & 571.6547 & 0.037879 & 562.2263 \\
\hline 0.040713 & 655.1829 & 0.037833 & 577.1534 & 0.044048 & 566.5449 \\
\hline 0.046103 & 658.9309 & 0.044396 & 581.7259 & 0.049389 & 569.7708 \\
\hline 0.052463 & 662.8617 & 0.050362 & 585.1929 & 0.054418 & 572.5128 \\
\hline 0.058669 & 665.9534 & 0.055515 & 587.9114 & 0.06079 & 575.601 \\
\hline 0.062993 & 667.9966 & 0.061553 & 590.7589 & 0.065971 & 577.899 \\
\hline 0.069382 & 670.9146 & 0.067447 & 593.3175 & 0.072133 & 580.4217 \\
\hline 0.074184 & 672.8306 & 0.073355 & 595.6232 & 0.076965 & 582.2879 \\
\hline 0.079699 & 674.8859 & 0.078184 & 597.4908 & 0.083476 & 584.7247 \\
\hline 0.085485 & 677.0021 & 0.082963 & 599.2427 & 0.087872 & 586.3683 \\
\hline 0.08986 & 678.4141 & 0.087777 & 600.9603 & 0.094615 & 588.6631 \\
\hline 0.096971 & 680.4915 & 0.09442 & 603.1564 & 0.099832 & 590.3282 \\
\hline 0.102471 & 682.006 & 0.100091 & 604.9431 & 0.10728 & 592.5599 \\
\hline 0.149118 & 692.4185 & 0.10715 & 607.0453 & 0.156335 & 605.211 \\
\hline 0.200096 & 700.8367 & 0.15911 & 619.6735 & 0.20701 & 616.8854 \\
\hline 0.246875 & 706.8791 & 0.210537 & 629.8205 & 0.260607 & 627.9705 \\
\hline 0.295272 & 712.7815 & 0.264807 & 639.517 & 0.312212 & 637.6506 \\
\hline 0.353302 & 718.0992 & 0.316479 & 648.1444 & 0.364733 & 647.4196 \\
\hline 0.399486 & 722.7275 & 0.367013 & 656.2806 & 0.415183 & 656.7446 \\
\hline 0.446316 & 726.4294 & 0.418915 & 664.4807 & 0.465851 & 666.0242 \\
\hline 0.496765 & 730.7949 & 0.467219 & 673.0191 & 0.515539 & 675.0664 \\
\hline 0.552089 & 735.9099 & 0.519315 & 681.175 & 0.56697 & 684.1724 \\
\hline 0.599964 & 738.435 & 0.572174 & 690.4234 & 0.620217 & 694.2511 \\
\hline
\end{tabular}




\begin{tabular}{|l|l|l|l|l|l|}
\hline 0.649147 & 742.175 & 0.620593 & 699.5052 & 0.670493 & 703.5922 \\
\hline 0.697787 & 745.9285 & 0.672553 & 708.7762 & 0.720253 & 712.3759 \\
\hline 0.74539 & 750.2803 & 0.723555 & 717.896 & 0.772113 & 723.0559 \\
\hline 0.804405 & 752.45 & 0.775198 & 727.692 & 0.823297 & 732.4781 \\
\hline 0.848037 & 756.0748 & 0.824661 & 733.9395 & 0.872678 & 740.59 \\
\hline 0.896133 & 759.2975 & 0.875973 & 749.8828 & 0.921559 & 748.8166 \\
\hline 0.957317 & 763.7646 & 0.923103 & 760.9373 & 0.969655 & 760.5616 \\
\hline 0.928383 & 761.7976 & 0.971464 & 770.761 & 0.923136 & 749.0519 \\
\hline 0.864214 & 760.2758 & 0.92432 & 761.1854 & 0.873434 & 739.927 \\
\hline 0.8135 & 756.5492 & 0.871856 & 748.347 & 0.822861 & 730.0485 \\
\hline 0.77439 & 755.7045 & 0.819198 & 733.6837 & 0.771248 & 720.0308 \\
\hline 0.715228 & 751.5026 & 0.77083 & 727.3405 & 0.720042 & 708.8027 \\
\hline 0.677199 & 749.1099 & 0.720202 & 717.4515 & 0.671031 & 700.6623 \\
\hline 0.617611 & 746.2068 & 0.669645 & 707.4074 & 0.62175 & 691.9382 \\
\hline 0.567302 & 743.5198 & 0.618621 & 697.8549 & 0.57162 & 683.1346 \\
\hline 0.515339 & 738.8293 & 0.568914 & 688.3286 & 0.523909 & 675.5869 \\
\hline 0.468309 & 735.0803 & 0.522302 & 680.1564 & 0.473859 & 666.3657 \\
\hline 0.422677 & 731.1867 & 0.471623 & 671.5258 & 0.423838 & 656.7089 \\
\hline 0.367177 & 725.6696 & 0.420117 & 661.3842 & 0.370495 & 647.728 \\
\hline 0.316934 & 720.608 & 0.367992 & 653.1938 & 0.320598 & 638.8817 \\
\hline 0.268199 & 714.4371 & 0.317695 & 645.0954 & 0.270874 & 629.9747 \\
\hline 0.219655 & 707.7146 & 0.26978 & 637.3734 & 0.219908 & 619.3949 \\
\hline 0.174507 & 700.4185 & 0.21854 & 631.3325 & 0.170744 & 608.5022 \\
\hline 0.123581 & 689.4715 & & & 0.124538 & 597.1846 \\
\hline 0.074956 & 674.3414 & & & 0.076507 & 582.131 \\
\hline 0.02739 & 641.9386 & & & 0.02658 & 551.5751 \\
\hline 0.017382 & 623.8947 & & & 0.01919 & 540.7043 \\
\hline & & & & & \\
\hline & & & & & \\
\hline
\end{tabular}

DMF resolvated samples

Table S26. $\mathrm{N}_{2}$ isotherm data for DMF resolvated IRMOF-3 samples used.

\begin{tabular}{|l|l|l|l|l|l|}
\hline $\begin{array}{l}\text { DMF } \\
\text { resolvation 1 }\end{array}$ & $\begin{array}{l}\text { DMF } \\
\text { resolvation 2 }\end{array}$ & & $\begin{array}{l}\text { DMF } \\
\text { resolvation 3 }\end{array}$ & \\
\hline $\mathrm{P} \mathrm{P}_{0}$ & $\mathrm{~N}_{2}$ uptake $\left(\mathrm{cc} \mathrm{g}^{-1}\right)$ & $\mathrm{P} / \mathrm{P}_{0}$ & $\begin{array}{l}\mathrm{N}_{2} \text { uptake } \\
\left(\mathrm{cc} \mathrm{g} \mathrm{g}^{-1}\right)\end{array}$ & $\mathrm{P} / \mathrm{P}_{0}$ & $\begin{array}{l}\mathrm{N}_{2} \text { uptake } \\
\left(\mathrm{cc} \mathrm{g}^{-1}\right)\end{array}$ \\
\hline 0.001662 & 180.1611 & 0.000885 & 128.5276 & 0.00162 & 207.5796 \\
\hline 0.002154 & 218.9357 & 0.001655 & 203.3457 & 0.002144 & 250.2977 \\
\hline 0.002199 & 222.8566 & 0.002029 & 230.3619 & 0.002209 & 254.947 \\
\hline 0.002279 & 227.1467 & 0.002173 & 238.1408 & 0.002296 & 260.0791 \\
\hline 0.002353 & 231.8916 & 0.002353 & 246.6368 & 0.002434 & 265.781 \\
\hline 0.002456 & 237.336 & 0.002591 & 255.3613 & 0.002565 & 271.8167 \\
\hline 0.003176 & 265.8267 & 0.002915 & 265.0928 & 0.003016 & 286.5162 \\
\hline 0.004 & 288.2216 & 0.003325 & 274.6969 & 0.004062 & 309.091 \\
\hline 0.005493 & 307.8072 & 0.004455 & 292.7894 & 0.005086 & 322.5855 \\
\hline
\end{tabular}




\begin{tabular}{|c|c|c|c|c|c|}
\hline 0.011897 & 339.5245 & 0.005361 & 302.4174 & 0.011168 & 357.1743 \\
\hline 0.016529 & 348.5803 & 0.01299 & 334.7081 & 0.016204 & 368.0979 \\
\hline 0.020684 & 353.9413 & 0.017826 & 342.5648 & 0.020724 & 374.2558 \\
\hline 0.025412 & 358.4455 & 0.023403 & 348.5971 & 0.026115 & 379.673 \\
\hline 0.03111 & 362.5414 & 0.029384 & 353.3444 & 0.031928 & 384.2192 \\
\hline 0.037559 & 366.1274 & 0.035307 & 357.1951 & 0.038635 & 388.4137 \\
\hline 0.044176 & 369.1537 & 0.041553 & 360.6304 & 0.045364 & 391.9648 \\
\hline 0.0495 & 371.1823 & 0.048145 & 363.8331 & 0.051446 & 394.8297 \\
\hline 0.054875 & 373.1438 & 0.053816 & 366.2299 & 0.056387 & 396.9683 \\
\hline 0.061662 & 375.2206 & 0.058422 & 368.0753 & 0.062527 & 399.396 \\
\hline 0.065904 & 376.416 & 0.065144 & 370.5275 & 0.067875 & 401.3583 \\
\hline 0.071368 & 377.9843 & 0.070275 & 372.4059 & 0.073753 & 403.4332 \\
\hline 0.077162 & 379.4887 & 0.075744 & 374.2612 & 0.079886 & 405.4549 \\
\hline 0.082941 & 380.8139 & 0.079703 & 375.6277 & 0.08426 & 406.887 \\
\hline 0.087463 & 381.8527 & 0.086122 & 377.7592 & 0.089536 & 408.5668 \\
\hline 0.092875 & 382.9979 & 0.090044 & 379.1548 & 0.094048 & 409.9361 \\
\hline 0.099147 & 384.1296 & 0.096744 & 381.2909 & 0.099643 & 411.5536 \\
\hline 0.105265 & 385.2043 & 0.102091 & 382.9609 & 0.106851 & 413.5122 \\
\hline 0.147743 & 392.1646 & 0.10805 & 384.8068 & 0.156429 & 425.4857 \\
\hline 0.202677 & 398.6947 & 0.161161 & 398.3148 & 0.209503 & 437.0048 \\
\hline 0.252684 & 403.9098 & 0.215107 & 410.862 & 0.261573 & 448.3271 \\
\hline 0.296368 & 408.3912 & 0.267189 & 422.6899 & 0.313905 & 458.8164 \\
\hline 0.353096 & 413.0034 & 0.319559 & 434.3219 & 0.365307 & 469.2386 \\
\hline 0.402022 & 417.8574 & 0.368402 & 445.4304 & 0.415445 & 479.6883 \\
\hline 0.448684 & 421.641 & 0.418649 & 456.8078 & 0.466462 & 490.1745 \\
\hline 0.496147 & 425.9421 & 0.468291 & 468.1273 & 0.517115 & 500.632 \\
\hline 0.546633 & 430.5416 & 0.520316 & 481.3311 & 0.563896 & 513.161 \\
\hline 0.595956 & 433.597 & 0.572606 & 494.617 & 0.614223 & 525.0263 \\
\hline 0.647809 & 437.4732 & 0.624429 & 507.079 & 0.66723 & 536.6924 \\
\hline 0.702471 & 441.9353 & 0.675367 & 521.1078 & 0.719918 & 548.5602 \\
\hline 0.750824 & 445.839 & 0.726233 & 534.9535 & 0.770456 & 560.0189 \\
\hline 0.801464 & 449.3042 & 0.775249 & 548.2406 & 0.818944 & 571.5144 \\
\hline 0.849677 & 453.4377 & 0.827201 & 563.538 & 0.868987 & 583.0411 \\
\hline 0.896214 & 457.5089 & 0.876023 & 577.2838 & 0.918907 & 594.1128 \\
\hline 0.946192 & 461.2641 & 0.922794 & 590.9225 & 0.967112 & 607.9591 \\
\hline 0.926023 & 460.3612 & 0.971299 & 603.9374 & 0.923768 & 596.1741 \\
\hline 0.863714 & 456.92 & 0.921945 & 590.3328 & 0.873732 & 583.3185 \\
\hline 0.827008 & 454.5021 & 0.870201 & 574.0363 & 0.823442 & 570.0721 \\
\hline 0.769206 & 452.7374 & 0.818097 & 558.8508 & 0.772948 & 556.9815 \\
\hline 0.729243 & 450.3658 & 0.770485 & 545.3809 & 0.7205 & 544.258 \\
\hline 0.665942 & 445.8383 & 0.719892 & 530.6158 & 0.66957 & 533.1418 \\
\hline 0.629699 & 443.0821 & 0.669307 & 515.4485 & 0.620566 & 522.4835 \\
\hline 0.567868 & 439.5304 & 0.619348 & 500.6916 & 0.571649 & 512.1232 \\
\hline
\end{tabular}




\begin{tabular}{|l|l|l|l|l|l|}
\hline 0.516861 & 435.4732 & 0.56972 & 488.4525 & 0.522311 & 500.3482 \\
\hline 0.468677 & 431.9687 & 0.523597 & 477.1262 & 0.472384 & 489.9293 \\
\hline 0.416515 & 426.865 & 0.470033 & 464.0813 & 0.42112 & 479.7355 \\
\hline 0.379 & 423.7849 & 0.421355 & 452.9155 & 0.368977 & 469.954 \\
\hline 0.315441 & 417.0367 & 0.367603 & 440.6591 & 0.317509 & 459.8841 \\
\hline 0.266177 & 412.2584 & 0.316846 & 429.355 & 0.270605 & 450.6246 \\
\hline 0.218184 & 406.478 & 0.266973 & 420.7146 & 0.218498 & 439.3749 \\
\hline 0.17364 & 400.5197 & 0.218972 & 412.4112 & 0.171594 & 429.1576 \\
\hline 0.120132 & 392.0191 & & & 0.1236 & 418.1889 \\
\hline 0.071029 & 381.0157 & & & 0.074058 & 404.2661 \\
\hline 0.027529 & 362.3257 & & & 0.029596 & 383.3769 \\
\hline 0.017846 & 352.6046 & & & 0.018907 & 372.7968 \\
\hline
\end{tabular}

DMSO resolvated samples

Table S27. $\mathrm{N}_{2}$ isotherm data for DMSO resolvated IRMOF-3 samples used.

\begin{tabular}{|l|l|l|l|l|l|}
\hline $\begin{array}{l}\text { DMSO } \\
\text { resolvation 1 }\end{array}$ & $\begin{array}{l}\text { DMSO } \\
\text { resolvation 2 }\end{array}$ & DMSO & \\
\hline $\mathrm{P} / \mathrm{P}_{0}$ & $\mathrm{~N}_{2}$ uptake $\left(\mathrm{cc} \mathrm{g}^{-1}\right)$ & $\mathrm{P} / \mathrm{P}_{0}$ & $\begin{array}{l}\mathrm{N}_{2} \text { uptake } \\
\left(\mathrm{cc} \mathrm{g}^{-1}\right)\end{array}$ & $\mathrm{P} \mathrm{P}_{0}$ & $\begin{array}{l}\mathrm{N}_{2} \text { uptake } \\
\left(\mathrm{cc} \mathrm{g}^{-1}\right)\end{array}$ \\
\hline 0.001625 & 116.3307 & 0.001101 & 100.6516 & 0.001715 & 152.5715 \\
\hline 0.002162 & 141.1628 & 0.001511 & 129.1903 & 0.002107 & 172.8787 \\
\hline 0.002184 & 143.422 & 0.002109 & 158.8034 & 0.002216 & 177.0995 \\
\hline 0.00225 & 145.8737 & 0.002253 & 163.848 & 0.002369 & 181.7205 \\
\hline 0.002346 & 148.5828 & 0.002454 & 169.2408 & 0.002543 & 186.7786 \\
\hline 0.002426 & 151.564 & 0.002706 & 175.0832 & 0.002768 & 192.1293 \\
\hline 0.00325 & 170.7839 & 0.003037 & 181.3358 & 0.003074 & 198.0708 \\
\hline 0.00439 & 186.5834 & 0.003498 & 187.7246 & 0.004672 & 217.3727 \\
\hline 0.005132 & 192.5459 & 0.004066 & 194.2263 & 0.005333 & 222.6763 \\
\hline 0.011691 & 214.2551 & 0.005585 & 205.4214 & 0.01151 & 244.3525 \\
\hline 0.01686 & 220.7427 & 0.01019 & 221.1301 & 0.015623 & 250.5002 \\
\hline 0.020485 & 223.9988 & 0.015206 & 228.4626 & 0.020883 & 255.6029 \\
\hline 0.025184 & 226.9503 & 0.021036 & 233.4706 & 0.027329 & 260.0302 \\
\hline 0.030757 & 229.5774 & 0.027477 & 237.2262 & 0.033832 & 263.5125 \\
\hline 0.036875 & 231.8846 & 0.033839 & 240.0931 & 0.040394 & 266.4542 \\
\hline 0.043125 & 233.8023 & 0.041071 & 242.7318 & 0.046984 & 269.0137 \\
\hline 0.04864 & 235.3273 & 0.047663 & 244.786 & 0.052739 & 271.0836 \\
\hline 0.055191 & 236.8124 & 0.0535 & 246.413 & 0.057745 & 272.7734 \\
\hline 0.060971 & 238.1046 & 0.058429 & 247.6797 & 0.062796 & 274.3494 \\
\hline 0.067228 & 239.2691 & 0.063301 & 248.8706 & 0.068827 & 276.1478 \\
\hline 0.072985 & 240.3227 & 0.06908 & 250.1861 & 0.074945 & 277.8433 \\
\hline 0.077934 & 241.231 & 0.075025 & 251.4348 & 0.079871 & 279.2115 \\
\hline 0.082529 & 241.9762 & 0.080264 & 252.5608 & 0.084224 & 280.3581 \\
\hline 0.088096 & 242.8038 & 0.086417 & 253.8288 & 0.089187 & 281.6414 \\
\hline
\end{tabular}




\begin{tabular}{|c|c|c|c|c|c|}
\hline 0.091816 & 243.37 & 0.092275 & 254.9991 & 0.095291 & 283.1702 \\
\hline 0.097743 & 244.1431 & 0.098219 & 256.145 & 0.103349 & 284.9993 \\
\hline 0.102949 & 244.785 & 0.10538 & 257.4633 & 0.109235 & 286.283 \\
\hline 0.147103 & 248.8019 & 0.109266 & 258.2541 & 0.1593 & 296.2751 \\
\hline 0.201022 & 252.8054 & 0.161809 & 265.9125 & 0.212242 & 306.203 \\
\hline 0.250066 & 255.5191 & 0.21451 & 272.7506 & 0.26384 & 315.4551 \\
\hline 0.298375 & 258.0419 & 0.266649 & 279.2921 & 0.316267 & 324.4273 \\
\hline 0.347515 & 260.5659 & 0.319444 & 285.7021 & 0.366768 & 333.6887 \\
\hline 0.396155 & 262.5364 & 0.370396 & 292.0236 & 0.415983 & 342.5959 \\
\hline 0.445287 & 264.824 & 0.421197 & 298.2267 & 0.465757 & 352.3089 \\
\hline 0.496552 & 266.6354 & 0.471717 & 304.5285 & 0.517123 & 361.651 \\
\hline 0.550015 & 268.8653 & 0.522957 & 311.7137 & 0.568902 & 372.3114 \\
\hline 0.596669 & 271.3735 & 0.573542 & 318.5832 & 0.619214 & 382.5042 \\
\hline 0.646794 & 273.0032 & 0.625106 & 325.9982 & 0.669854 & 392.6416 \\
\hline 0.698118 & 274.0413 & 0.674913 & 333.2522 & 0.720609 & 402.7234 \\
\hline 0.75028 & 275.3335 & 0.725794 & 340.5952 & 0.772192 & 412.8546 \\
\hline 0.798787 & 276.5626 & 0.774443 & 348.3696 & 0.821589 & 422.7591 \\
\hline 0.848692 & 278.3721 & 0.823955 & 356.1399 & 0.872446 & 433.3597 \\
\hline 0.904979 & 279.0742 & 0.873346 & 364.5073 & 0.920462 & 445.0219 \\
\hline 0.949684 & 282.2099 & 0.921916 & 373.7509 & 0.965361 & 459.4611 \\
\hline 0.928706 & 281.1589 & 0.970788 & 385.8022 & 0.926667 & 448.502 \\
\hline 0.867265 & 280.1439 & 0.925946 & 375.1129 & 0.874052 & 433.8221 \\
\hline 0.826964 & 280.1258 & 0.873447 & 364.5311 & 0.821138 & 421.0546 \\
\hline 0.770361 & 279.0532 & 0.820688 & 354.9453 & 0.770717 & 409.9062 \\
\hline 0.714927 & 279.2856 & 0.770189 & 346.5994 & 0.720594 & 399.739 \\
\hline 0.675993 & 278.4192 & 0.720432 & 338.2409 & 0.669657 & 389.402 \\
\hline 0.615456 & 276.8795 & 0.670221 & 329.9955 & 0.619367 & 379.6011 \\
\hline 0.569184 & 275.3206 & 0.619866 & 322.3939 & 0.569506 & 370.6825 \\
\hline 0.528537 & 274.2235 & 0.570188 & 315.2271 & 0.522056 & 361.6745 \\
\hline 0.469927 & 271.6785 & 0.524288 & 309.2653 & 0.474077 & 352.6 \\
\hline 0.413361 & 269.7094 & 0.469896 & 301.7328 & 0.420735 & 343.0904 \\
\hline 0.379875 & 268.1011 & 0.418599 & 295.354 & 0.369384 & 334.5185 \\
\hline 0.318912 & 265.3591 & 0.367121 & 289.0394 & 0.319479 & 325.8983 \\
\hline 0.267309 & 262.3832 & 0.318796 & 283.171 & 0.268404 & 317.2887 \\
\hline 0.218302 & 259.5263 & 0.269305 & 279.5297 & 0.217605 & 308.2139 \\
\hline 0.173743 & 256.2175 & 0.221217 & 274.9926 & 0.170119 & 299.479 \\
\hline 0.120228 & 251.2956 & & & 0.122358 & 290.3051 \\
\hline 0.071375 & 244.9935 & & & 0.07283 & 279.1377 \\
\hline 0.028221 & 233.9145 & & & 0.027489 & 262.6388 \\
\hline 0.018434 & 228.0562 & & & 0.016996 & 254.519 \\
\hline
\end{tabular}




\section{THF RSE samples}

Table S28. $\mathrm{N}_{2}$ isotherm data for THF RSE IRMOF-3 samples used.

\begin{tabular}{|c|c|c|c|c|c|}
\hline $\begin{array}{l}\text { THF RSE } \\
1\end{array}$ & & $\begin{array}{l}\text { THF RSE } \\
2\end{array}$ & & $\begin{array}{l}\text { THF RSE } \\
3\end{array}$ & \\
\hline $\mathrm{P} / \mathrm{P}_{0}$ & $\mathrm{~N}_{2}$ uptake $\left(\mathrm{cc} \mathrm{g}^{-1}\right)$ & $\mathrm{P} / \mathrm{P}_{0}$ & $\begin{array}{l}\mathrm{N}_{2} \text { uptake (cc g- } \\
1 \text { ) }\end{array}$ & $\mathrm{P} / \mathrm{P}_{0}$ & $\begin{array}{l}\mathrm{N}_{2} \text { uptake (cc g } \\
1 \text { ) }\end{array}$ \\
\hline 0.002153 & 336.0491 & 0.000947 & 214.6897 & 0.001565 & 287.81 \\
\hline 0.002182 & 340.9833 & 0.001608 & 321.0983 & 0.002121 & 367.7562 \\
\hline 0.002233 & 346.5504 & 0.002093 & 373.3325 & 0.002188 & 373.268 \\
\hline 0.002292 & 352.7055 & 0.00213 & 378.2264 & 0.00224 & 379.5833 \\
\hline 0.002365 & 359.8325 & 0.002196 & 383.6106 & 0.002314 & 386.5822 \\
\hline 0.002452 & 367.5786 & 0.002269 & 389.5445 & 0.002388 & 394.1159 \\
\hline 0.003102 & 414.3866 & 0.002372 & 395.9398 & 0.003056 & 440.6198 \\
\hline 0.004182 & 454.4485 & 0.003121 & 435.0774 & 0.004198 & 485.5865 \\
\hline 0.005583 & 482.9213 & 0.004017 & 462.7153 & 0.005414 & 513.2454 \\
\hline 0.01094 & 529.1996 & 0.005229 & 487.1484 & 0.010917 & 566.1671 \\
\hline 0.016231 & 546.9528 & 0.010591 & 533.9703 & 0.015359 & 583.6472 \\
\hline 0.020063 & 554.7376 & 0.017451 & 555.4393 & 0.022026 & 598.4071 \\
\hline 0.025069 & 562.0879 & 0.020741 & 561.4924 & 0.026024 & 604.3971 \\
\hline 0.030623 & 568.238 & 0.025419 & 568.1292 & 0.031564 & 610.9189 \\
\hline 0.036841 & 573.4077 & 0.030891 & 574.0306 & 0.036711 & 615.8163 \\
\hline 0.043001 & 577.6481 & 0.03612 & 578.5205 & 0.042392 & 620.2519 \\
\hline 0.048942 & 581.0937 & 0.04215 & 582.7675 & 0.048458 & 624.3411 \\
\hline 0.05516 & 584.004 & 0.048511 & 586.7978 & 0.053835 & 627.7504 \\
\hline 0.061633 & 587.0252 & 0.054158 & 589.6902 & 0.059783 & 631.1309 \\
\hline 0.065677 & 588.589 & 0.060695 & 592.6429 & 0.065486 & 634.1714 \\
\hline 0.070756 & 590.4254 & 0.066284 & 594.8123 & 0.070893 & 636.9054 \\
\hline 0.075515 & 592.1821 & 0.072138 & 597.0448 & 0.075802 & 639.1415 \\
\hline 0.081076 & 593.8859 & 0.076523 & 598.5518 & 0.080578 & 641.0356 \\
\hline 0.086798 & 595.4786 & 0.082655 & 600.6113 & 0.087171 & 643.4482 \\
\hline 0.090709 & 596.723 & 0.08845 & 602.3198 & 0.092689 & 645.3442 \\
\hline 0.098175 & 598.7194 & 0.093709 & 603.991 & 0.099074 & 647.3987 \\
\hline 0.102073 & 599.7921 & 0.099195 & 605.4869 & 0.102938 & 648.5342 \\
\hline 0.146971 & 608.6805 & 0.104307 & 606.9232 & 0.14536 & 659.7101 \\
\hline 0.201394 & 617.0623 & 0.146729 & 615.8678 & 0.199469 & 670.7612 \\
\hline 0.249548 & 622.6682 & 0.199014 & 624.8418 & 0.247668 & 678.7069 \\
\hline 0.296015 & 627.505 & 0.246996 & 630.5939 & 0.296593 & 685.585 \\
\hline 0.347417 & 632.471 & 0.296014 & 637.7493 & 0.353476 & 692.4227 \\
\hline 0.398884 & 637.7339 & 0.346184 & 643.0043 & 0.399383 & 697.3568 \\
\hline 0.448125 & 641.5112 & 0.396428 & 648.3101 & 0.448894 & 702.0233 \\
\hline 0.497293 & 645.4165 & 0.445768 & 654.7043 & 0.499021 & 706.8129 \\
\hline 0.549395 & 649.1943 & 0.496908 & 659.6954 & 0.550342 & 711.0496 \\
\hline 0.600212 & 653.4532 & 0.549745 & 668.0101 & 0.60015 & 715.4678 \\
\hline
\end{tabular}




\begin{tabular}{|l|l|l|l|l|l|}
\hline 0.648373 & 657.1551 & 0.59979 & 672.5937 & 0.649024 & 720.0297 \\
\hline 0.700818 & 661.161 & 0.649263 & 678.6118 & 0.698654 & 722.4406 \\
\hline 0.751102 & 663.2847 & 0.69936 & 686.5641 & 0.747794 & 726.05 \\
\hline 0.800322 & 667.193 & 0.750691 & 694.8319 & 0.7987 & 729.7762 \\
\hline 0.850241 & 671.8789 & 0.796183 & 701.6008 & 0.847121 & 732.8277 \\
\hline 0.906058 & 676.3328 & 0.851223 & 710.8892 & 0.897448 & 737.0994 \\
\hline 0.952788 & 680.3253 & 0.895319 & 717.3279 & 0.947767 & 741.6566 \\
\hline 0.916735 & 679.0518 & 0.957659 & 727.2491 & 0.926468 & 741.3436 \\
\hline 0.877245 & 680.3015 & 0.927995 & 726.7494 & 0.8667 & 736.1825 \\
\hline 0.813473 & 676.6527 & 0.866 & 715.7516 & 0.825028 & 734.703 \\
\hline 0.776092 & 674.5958 & 0.828198 & 712.1823 & 0.777281 & 732.1276 \\
\hline 0.713181 & 673.5366 & 0.770403 & 707.0701 & 0.716297 & 729.0649 \\
\hline 0.679543 & 671.5985 & 0.726865 & 706.207 & 0.665243 & 725.1905 \\
\hline 0.618027 & 667.2744 & 0.668388 & 700.4927 & 0.61703 & 722.4497 \\
\hline 0.578244 & 666.4128 & 0.617278 & 698.1651 & 0.56826 & 717.5941 \\
\hline 0.515597 & 660.9572 & 0.569127 & 690.5891 & 0.529139 & 716.0071 \\
\hline 0.467808 & 655.668 & 0.516871 & 683.4295 & 0.468006 & 708.5866 \\
\hline 0.428127 & 651.4841 & 0.478657 & 679.8327 & 0.420764 & 703.1791 \\
\hline 0.367509 & 645.1309 & 0.421664 & 672.9671 & 0.369377 & 697.2643 \\
\hline 0.318749 & 639.9587 & 0.370957 & 666.309 & 0.319539 & 690.8801 \\
\hline 0.267625 & 633.5225 & 0.322858 & 661.4533 & 0.268077 & 682.9757 \\
\hline 0.218078 & 626.2482 & 0.269507 & 652.84 & 0.21933 & 674.9806 \\
\hline 0.17253 & 618.3156 & 0.221246 & 644.1741 & 0.173245 & 665.8208 \\
\hline 0.12018 & 606.9327 & 0.172868 & 634.4782 & 0.121464 & 652.8097 \\
\hline 0.070887 & 591.3418 & 0.122793 & 619.7231 & 0.07337 & 635.6486 \\
\hline 0.028514 & 564.2675 & & & 0.022991 & 595.2245 \\
\hline 0.018705 & 549.4815 & & & 0.018378 & 585.9238 \\
\hline & & & & & \\
\hline
\end{tabular}

\section{DMF RSE samples}

Table S29. $\mathrm{N}_{2}$ isotherm data for DMF RSE IRMOF-3 samples used.

\begin{tabular}{|l|l|l|l|l|l|}
\hline DMF RSE 1 & & DMF RSE 2 & & DMF RSE 3 & \\
\hline $\mathrm{P} \mathrm{P}_{0}$ & $\begin{array}{l}\mathrm{N}_{2} \text { uptake }(\mathrm{cc} \\
\left.\mathrm{g}^{-1}\right)\end{array}$ & $\mathrm{P} / \mathrm{P}_{0}$ & $\begin{array}{l}\mathrm{N}_{2} \text { uptake }(\mathrm{cc} \\
\left.\mathrm{g}^{-1}\right)\end{array}$ & $\mathrm{P} \mathrm{P}_{0}$ & $\begin{array}{l}\mathrm{N}_{2} \text { uptake } \\
\mathrm{g}^{-1}\end{array}$ \\
\hline 0.001992 & 297.2275 & 0.000867 & 186.649 & 0.001609 & 278.1537 \\
\hline 0.002036 & 301.3062 & 0.001594 & 292.7418 & 0.002069 & 337.1375 \\
\hline 0.002095 & 305.9058 & 0.002027 & 344.1209 & 0.002114 & 342.563 \\
\hline 0.00216 & 310.9679 & 0.002042 & 348.9767 & 0.002188 & 348.3981 \\
\hline 0.002204 & 316.545 & 0.002108 & 354.3122 & 0.00224 & 354.8318 \\
\hline 0.00227 & 322.951 & 0.002181 & 360.1399 & 0.002329 & 362.1072 \\
\hline 0.003299 & 384.0099 & 0.002262 & 366.4507 & 0.003041 & 407.2222 \\
\hline 0.004313 & 416.1372 & 0.003378 & 422.8767 & 0.004294 & 450.3633 \\
\hline 0.005101 & 431.5091 & 0.004252 & 446.7949 & 0.005132 & 467.8469 \\
\hline
\end{tabular}




\begin{tabular}{|c|c|c|c|c|c|}
\hline 0.010561 & 478.1471 & 0.005031 & 461.9792 & 0.010702 & 518.3123 \\
\hline 0.015275 & 493.5019 & 0.010451 & 510.347 & 0.015441 & 534.926 \\
\hline 0.022449 & 506.0423 & 0.017091 & 531.1786 & 0.021834 & 547.2863 \\
\hline 0.026792 & 511.1758 & 0.020374 & 537.2101 & 0.026328 & 553.2234 \\
\hline 0.032025 & 515.99 & 0.02774 & 546.7379 & 0.031994 & 559.0228 \\
\hline 0.037703 & 520.1992 & 0.032793 & 551.5255 & 0.037415 & 563.4919 \\
\hline 0.043964 & 523.9042 & 0.037788 & 555.282 & 0.043141 & 567.4141 \\
\hline 0.04938 & 526.8033 & 0.043663 & 559.15 & 0.04937 & 571.1518 \\
\hline 0.055817 & 529.5624 & 0.048687 & 562.2364 & 0.054873 & 574.2006 \\
\hline 0.061677 & 532.0298 & 0.054974 & 564.9103 & 0.061333 & 577.4133 \\
\hline 0.065604 & 533.5961 & 0.061173 & 567.5782 & 0.067184 & 580.152 \\
\hline 0.070617 & 535.3784 & 0.065109 & 569.2931 & 0.071849 & 582.2767 \\
\hline 0.076507 & 537.3189 & 0.071829 & 571.6218 & 0.076944 & 584.2976 \\
\hline 0.081755 & 538.776 & 0.078226 & 573.816 & 0.082291 & 586.1708 \\
\hline 0.08717 & 540.3886 & 0.083324 & 575.1668 & 0.088662 & 588.2839 \\
\hline 0.093308 & 542.0037 & 0.089162 & 577.1969 & 0.093557 & 589.7249 \\
\hline 0.099496 & 543.4839 & 0.092225 & 578.023 & 0.099742 & 591.4318 \\
\hline 0.104138 & 544.5902 & 0.098725 & 579.8535 & 0.103242 & 592.2468 \\
\hline 0.147387 & 553.1906 & 0.10214 & 580.817 & 0.151664 & 603.6407 \\
\hline 0.200978 & 561.1253 & 0.147236 & 589.7182 & 0.201464 & 613.1317 \\
\hline 0.245271 & 566.5176 & 0.202092 & 598.2694 & 0.24634 & 619.5118 \\
\hline 0.302927 & 573.4507 & 0.248223 & 604.4965 & 0.300168 & 626.6739 \\
\hline 0.350183 & 578.6074 & 0.296616 & 610.2349 & 0.346008 & 632.1976 \\
\hline 0.399635 & 583.5928 & 0.347212 & 615.0975 & 0.404552 & 638.3534 \\
\hline 0.449336 & 588.0449 & 0.39573 & 621.5311 & 0.449636 & 642.3791 \\
\hline 0.495563 & 592.0809 & 0.447524 & 625.7798 & 0.497864 & 646.1415 \\
\hline 0.54511 & 596.2957 & 0.495601 & 632.2579 & 0.546931 & 650.3192 \\
\hline 0.602095 & 600.045 & 0.546491 & 637.3763 & 0.603013 & 654.706 \\
\hline 0.646621 & 604.1661 & 0.595714 & 643.4907 & 0.652732 & 657.694 \\
\hline 0.70262 & 609.1344 & 0.650196 & 650.9399 & 0.697726 & 662.1694 \\
\hline 0.749103 & 613.4664 & 0.698633 & 657.9734 & 0.750197 & 665.9512 \\
\hline 0.798242 & 616.4755 & 0.745755 & 665.0342 & 0.799998 & 669.3784 \\
\hline 0.847453 & 621.0927 & 0.795199 & 671.8312 & 0.851511 & 671.9625 \\
\hline 0.8963 & 625.7416 & 0.853037 & 678.9205 & 0.904605 & 676.2267 \\
\hline 0.945483 & 628.9581 & 0.895202 & 685.8105 & 0.949696 & 679.1222 \\
\hline 0.926405 & 629.5519 & 0.952291 & 694.8168 & 0.925756 & 677.3068 \\
\hline 0.866567 & 625.6659 & 0.929853 & 693.532 & 0.867063 & 673.9807 \\
\hline 0.82769 & 622.9211 & 0.864883 & 683.9901 & 0.827697 & 673.2714 \\
\hline 0.763502 & 621.8822 & 0.827816 & 679.2393 & 0.76385 & 669.0172 \\
\hline 0.728639 & 619.6847 & 0.762258 & 676.7507 & 0.727889 & 667.3616 \\
\hline 0.667764 & 614.5703 & 0.728422 & 674.9084 & 0.667913 & 662.8793 \\
\hline 0.614152 & 611.8513 & 0.679265 & 673.3261 & 0.628799 & 659.7817 \\
\hline 0.566086 & 607.9277 & 0.617917 & 665.9523 & 0.568942 & 655.5734 \\
\hline
\end{tabular}




\begin{tabular}{|l|l|l|l|l|l|}
\hline 0.515137 & 603.6722 & 0.570912 & 658.7189 & 0.516413 & 651.9185 \\
\hline 0.479346 & 601.4576 & 0.515468 & 653.5934 & 0.477833 & 648.7009 \\
\hline 0.416553 & 594.659 & 0.478378 & 650.1229 & 0.420364 & 643.5643 \\
\hline 0.366151 & 588.9484 & 0.418535 & 643.6967 & 0.366366 & 637.4416 \\
\hline 0.329514 & 584.645 & 0.366396 & 639.2495 & 0.320355 & 631.4934 \\
\hline 0.268851 & 576.7859 & 0.32158 & 633.5384 & 0.266668 & 624.1602 \\
\hline 0.219005 & 569.4714 & 0.271725 & 625.8121 & 0.219189 & 616.7059 \\
\hline 0.1735 & 561.9103 & 0.220806 & 617.1911 & 0.17501 & 608.4371 \\
\hline 0.120793 & 551.3548 & 0.174095 & 607.9672 & 0.123037 & 596.6381 \\
\hline 0.071676 & 537.0038 & 0.120546 & 593.2684 & 0.072954 & 580.4229 \\
\hline 0.029631 & 513.0324 & & & 0.02951 & 552.9354 \\
\hline 0.019552 & 500.2157 & & & 0.017925 & 535.5542 \\
\hline
\end{tabular}

DMSO RSE samples

Table S30. $\mathrm{N}_{2}$ isotherm data for DMSO RSE IRMOF-3 samples used.

\begin{tabular}{|l|l|l|l|l|l|}
\hline DMSO RSE 1 & & DMSO RSE 2 & & DMSO RSE 3 & \\
\hline${\mathrm{P} / \mathrm{P}_{0}}$ & $\begin{array}{l}\mathrm{N}_{2} \text { uptake } \\
\left(\mathrm{cc} \mathrm{g}^{-1}\right)\end{array}$ & $\mathrm{P} / \mathrm{P}_{0}$ & $\begin{array}{l}\mathrm{N}_{2} \text { uptake } \\
\left(\mathrm{cc} \mathrm{g}^{-1}\right)\end{array}$ & $\mathrm{P} / \mathrm{P}_{0}$ & $\begin{array}{l}\mathrm{N}_{2} \text { uptake } \\
\left(\mathrm{cc} \mathrm{g}^{-1}\right)\end{array}$ \\
\hline 0.002131 & 294.6091 & 0.000984 & 183.6272 & 0.001602 & 274.2739 \\
\hline 0.002138 & 298.6055 & 0.001557 & 256.9007 & 0.002158 & 340.1241 \\
\hline 0.002204 & 303.0772 & 0.002123 & 311.5657 & 0.002195 & 346.1451 \\
\hline 0.002262 & 308.0261 & 0.002174 & 316.6591 & 0.002277 & 352.6394 \\
\hline 0.002328 & 313.7605 & 0.002247 & 322.2112 & 0.002358 & 360.1766 \\
\hline 0.002408 & 319.9992 & 0.00235 & 328.3485 & 0.002462 & 368.2809 \\
\hline 0.003051 & 358.5619 & 0.002482 & 335.0712 & 0.003004 & 399.4894 \\
\hline 0.004269 & 396.3096 & 0.00307 & 360.4033 & 0.004568 & 447.7699 \\
\hline 0.005087 & 411.5046 & 0.004062 & 386.3524 & 0.005377 & 462.5552 \\
\hline 0.011101 & 457.8983 & 0.00506 & 402.9339 & 0.011688 & 511.6286 \\
\hline 0.016209 & 472.2386 & 0.011421 & 447.4154 & 0.016331 & 525.7411 \\
\hline 0.02242 & 481.9895 & 0.016922 & 461.1919 & 0.020558 & 533.7332 \\
\hline 0.026908 & 486.8136 & 0.020719 & 467.1249 & 0.025549 & 540.6367 \\
\hline 0.03209 & 491.3204 & 0.026022 & 473.1832 & 0.031586 & 546.9406 \\
\hline 0.037761 & 495.1809 & 0.031963 & 478.285 & 0.037423 & 551.6746 \\
\hline 0.043877 & 498.6788 & 0.037523 & 482.0859 & 0.043482 & 555.8147 \\
\hline 0.049621 & 501.3914 & 0.044052 & 485.6331 & 0.050023 & 559.7631 \\
\hline 0.05597 & 504.0538 & 0.049627 & 488.4011 & 0.055496 & 562.8157 \\
\hline 0.062283 & 506.2812 & 0.055716 & 490.8509 & 0.061763 & 565.9904 \\
\hline 0.065844 & 507.7122 & 0.062318 & 493.1158 & 0.067881 & 568.931 \\
\hline 0.070727 & 509.2171 & 0.066306 & 494.588 & 0.071182 & 570.6154 \\
\hline 0.075989 & 510.7322 & 0.07169 & 496.2925 & 0.076952 & 572.9882 \\
\hline 0.081521 & 512.3406 & 0.075986 & 497.4715 & 0.080912 & 574.4736 \\
\hline 0.087411 & 513.7351 & 0.081935 & 499.4141 & 0.087283 & 576.699 \\
\hline 0.091403 & 514.7025 & 0.088913 & 501.0884 & 0.092407 & 578.1663 \\
\hline & & & & & \\
\hline
\end{tabular}




\begin{tabular}{|c|c|c|c|c|c|}
\hline 0.098519 & 516.2456 & 0.092049 & 501.7493 & 0.09906 & 580.207 \\
\hline 0.103423 & 517.5253 & 0.098725 & 503.494 & 0.105148 & 581.8795 \\
\hline 0.148883 & 525.3434 & 0.102566 & 504.2838 & 0.147911 & 592.7761 \\
\hline 0.197263 & 531.3251 & 0.147537 & 512.1932 & 0.201798 & 604.1666 \\
\hline 0.252087 & 537.5227 & 0.195408 & 518.27 & 0.248217 & 612.1457 \\
\hline 0.300854 & 542.041 & 0.245748 & 524.2439 & 0.300539 & 620.3532 \\
\hline 0.348716 & 546.1488 & 0.297093 & 529.0074 & 0.348352 & 627.3945 \\
\hline 0.398446 & 549.9331 & 0.34506 & 534.7039 & 0.398011 & 634.2961 \\
\hline 0.450153 & 553.3713 & 0.395737 & 539.119 & 0.445943 & 640.746 \\
\hline 0.498475 & 556.4057 & 0.449587 & 545.0054 & 0.504406 & 648.2607 \\
\hline 0.549103 & 560.2529 & 0.496365 & 549.6652 & 0.550572 & 653.7989 \\
\hline 0.59584 & 562.9208 & 0.548019 & 554.4058 & 0.597043 & 659.3956 \\
\hline 0.646169 & 565.9712 & 0.600025 & 562.1267 & 0.649765 & 665.0773 \\
\hline 0.698767 & 570.3647 & 0.646017 & 567.1153 & 0.700174 & 670.9545 \\
\hline 0.751745 & 573.1523 & 0.696327 & 572.9683 & 0.748076 & 676.7368 \\
\hline 0.800197 & 576.4363 & 0.747511 & 581.7336 & 0.797847 & 681.4171 \\
\hline 0.847271 & 579.9305 & 0.796932 & 587.6982 & 0.849242 & 686.7862 \\
\hline 0.904321 & 584.1713 & 0.853485 & 594.999 & 0.896521 & 692.592 \\
\hline 0.950767 & 587.7524 & 0.899696 & 604.577 & 0.950259 & 697.8272 \\
\hline 0.927267 & 586.9693 & 0.946981 & 610.2365 & 0.9254 & 697.6157 \\
\hline 0.866173 & 583.9642 & 0.927848 & 607.9395 & 0.87218 & 691.3291 \\
\hline 0.82342 & 583.9773 & 0.866433 & 598.9949 & 0.829158 & 687.1118 \\
\hline 0.77731 & 581.8821 & 0.826692 & 595.9246 & 0.779773 & 683.6642 \\
\hline 0.716239 & 579.0092 & 0.773767 & 593.9343 & 0.717521 & 677.549 \\
\hline 0.676872 & 578.3063 & 0.727063 & 593.557 & 0.677762 & 672.4506 \\
\hline 0.618173 & 574.4708 & 0.668748 & 588.5503 & 0.618105 & 665.451 \\
\hline 0.566509 & 571.065 & 0.619224 & 583.6949 & 0.56763 & 660.5938 \\
\hline 0.528595 & 570.1062 & 0.569061 & 577.5218 & 0.514996 & 654.4188 \\
\hline 0.46799 & 564.8257 & 0.513845 & 571.751 & 0.478641 & 650.0303 \\
\hline 0.420618 & 560.5903 & 0.476931 & 568.6789 & 0.420661 & 641.7841 \\
\hline 0.378317 & 557.0984 & 0.418594 & 564.1545 & 0.366292 & 633.4677 \\
\hline 0.316706 & 551.0197 & 0.364979 & 558.3785 & 0.329329 & 628.1516 \\
\hline 0.266472 & 545.535 & 0.321073 & 553.1884 & 0.269664 & 617.6184 \\
\hline 0.217735 & 539.913 & 0.271013 & 546.3282 & 0.219167 & 608.5179 \\
\hline 0.171771 & 533.2518 & 0.219785 & 538.8369 & 0.174276 & 599.1159 \\
\hline 0.119172 & 523.4331 & 0.17098 & 529.3345 & 0.122273 & 586.1127 \\
\hline 0.071581 & 510.7324 & & & 0.072131 & 568.6362 \\
\hline 0.029113 & 488.1494 & & & 0.028167 & 539.5181 \\
\hline 0.019129 & 475.9723 & & & 0.016605 & 520.8652 \\
\hline
\end{tabular}


FJI-1

Pristine samples

Table S31. $\mathrm{N}_{2}$ isotherm data for pristine FJl-1 samples used. Samples are labelled with their BET surface areas, given in parentheses.

\begin{tabular}{|c|c|c|c|c|c|}
\hline $\begin{array}{l}\text { Pristine } \\
(4736.240)\end{array}$ & & $\begin{array}{l}\text { Pristine } \\
(4922.309)\end{array}$ & & $\begin{array}{l}\text { Pristine } \\
\text { (4512.863) }\end{array}$ & \\
\hline $\mathrm{P} / \mathrm{P}_{0}$ & $\begin{array}{l}\mathrm{N}_{2} \text { uptake } \\
\left(\mathrm{cc} \mathrm{g}^{-1}\right)\end{array}$ & $\mathrm{P} / \mathrm{P}_{0}$ & $\begin{array}{l}\mathrm{N}_{2} \text { uptake } \\
\left(\mathrm{cc} \mathrm{g} \mathrm{g}^{-1}\right)\end{array}$ & $\mathrm{P} / \mathrm{P}_{0}$ & $\begin{array}{l}\mathrm{N}_{2} \text { uptake } \\
\left(\mathrm{cc} \mathrm{g}^{-1}\right)\end{array}$ \\
\hline 0.001526 & 207.8972 & 0.000972 & 156.3222 & 0.001073 & 144.9805 \\
\hline 0.002178 & 294.6489 & 0.001502 & 198.9866 & 0.001628 & 190.612 \\
\hline 0.002149 & 300.6988 & 0.00204 & 245.9087 & 0.002138 & 237.2355 \\
\hline 0.002149 & 307.7027 & 0.002076 & 250.1191 & 0.002175 & 241.4341 \\
\hline 0.002192 & 315.0204 & 0.002143 & 254.8657 & 0.002249 & 246.038 \\
\hline 0.002228 & 322.9532 & 0.002157 & 260.3267 & 0.002308 & 251.3225 \\
\hline 0.003102 & 495.9367 & 0.002209 & 266.004 & 0.002353 & 257.0678 \\
\hline 0.004255 & 614.345 & 0.003004 & 394.3553 & 0.003004 & 360.7419 \\
\hline 0.005194 & 652.0475 & 0.004065 & 590.6368 & 0.004224 & 546.3065 \\
\hline 0.011161 & 733.0936 & 0.005316 & 672.0308 & 0.005171 & 598.577 \\
\hline 0.016047 & 771.396 & 0.011928 & 769.8162 & 0.012436 & 696.7001 \\
\hline 0.020324 & 801.3021 & 0.015176 & 794.8428 & 0.015365 & 718.8778 \\
\hline 0.025854 & 839.6839 & 0.020219 & 830.5734 & 0.020677 & 754.1662 \\
\hline 0.030196 & 870.2867 & 0.025285 & 866.1451 & 0.026115 & 789.9651 \\
\hline 0.035454 & 909.2764 & 0.030447 & 902.408 & 0.03099 & 822.0793 \\
\hline 0.040297 & 949.9116 & 0.035528 & 939.8503 & 0.035288 & 851.2169 \\
\hline 0.046945 & 1005.8856 & 0.040954 & 984.9285 & 0.041687 & 901.2347 \\
\hline 0.050176 & 1028.7483 & 0.045792 & 1028.7626 & 0.045438 & 933.7219 \\
\hline 0.055148 & 1050.583 & 0.051668 & 1070.123 & 0.051645 & 973.1037 \\
\hline 0.060929 & 1066.6178 & 0.055909 & 1089.4194 & 0.055307 & 989.0392 \\
\hline 0.065406 & 1075.7897 & 0.062543 & 1107.303 & 0.062704 & 1007.8018 \\
\hline 0.070278 & 1083.7483 & 0.066033 & 1114.1071 & 0.065323 & 1012.8107 \\
\hline 0.076066 & 1091.3788 & 0.072115 & 1123.412 & 0.070539 & 1020.6096 \\
\hline 0.082757 & 1098.7362 & 0.076489 & 1128.7545 & 0.075569 & 1026.5918 \\
\hline 0.090473 & 1106.0057 & 0.082144 & 1134.5321 & 0.081081 & 1032.5103 \\
\hline 0.09816 & 1112.354 & 0.087541 & 1139.3625 & 0.086637 & 1037.5477 \\
\hline 0.104005 & 1116.7386 & 0.092394 & 1143.3624 & 0.092037 & 1041.9736 \\
\hline 0.150456 & 1141.6581 & 0.098513 & 1147.9969 & 0.098554 & 1046.4351 \\
\hline 0.200087 & 1161.9001 & 0.147206 & 1172.1654 & 0.150599 & 1070.3871 \\
\hline 0.250929 & 1179.0438 & 0.196414 & 1187.259 & 0.198559 & 1083.8384 \\
\hline 0.304809 & 1195.4268 & 0.247022 & 1199.136 & 0.250352 & 1095.203 \\
\hline 0.356783 & 1210.5081 & 0.30053 & 1210.2786 & 0.298563 & 1103.381 \\
\hline 0.408141 & 1225.054 & 0.345159 & 1217.8383 & 0.347959 & 1111.5244 \\
\hline 0.461619 & 1240.7404 & 0.40176 & 1226.7402 & 0.395601 & 1118.1348 \\
\hline
\end{tabular}




\begin{tabular}{|l|l|l|l|l|l|}
\hline 0.514997 & 1255.5475 & 0.445888 & 1233.3501 & 0.450834 & 1125.2118 \\
\hline 0.564571 & 1270.5828 & 0.501134 & 1241.2034 & 0.496627 & 1130.17 \\
\hline 0.614045 & 1285.0652 & 0.550129 & 1249.0629 & 0.550506 & 1135.8177 \\
\hline 0.666134 & 1300.1024 & 0.59542 & 1254.7095 & 0.596298 & 1139.1899 \\
\hline 0.716274 & 1314.3259 & 0.647235 & 1261.1316 & 0.651117 & 1144.9194 \\
\hline 0.768333 & 1329.6711 & 0.695141 & 1267.8237 & 0.700475 & 1149.8992 \\
\hline 0.816023 & 1343.7367 & 0.747714 & 1273.2496 & 0.747726 & 1154.2919 \\
\hline 0.865583 & 1358.2772 & 0.79601 & 1280.0435 & 0.799888 & 1158.1755 \\
\hline 0.916662 & 1373.8811 & 0.852817 & 1288.5479 & 0.848899 & 1162.6521 \\
\hline 0.967368 & 1389.7856 & 0.896569 & 1296.1731 & 0.897118 & 1167.7919 \\
\hline 0.920867 & 1376.1389 & 0.944982 & 1313.7137 & 0.946588 & 1170.8273 \\
\hline 0.870512 & 1360.4611 & 0.928165 & 1311.2595 & 0.92907 & 1170.4009 \\
\hline 0.820286 & 1345.0776 & 0.868309 & 1301.9583 & 0.86908 & 1165.568 \\
\hline 0.770941 & 1330.3965 & 0.81712 & 1295.1616 & 0.828673 & 1161.4366 \\
\hline 0.721331 & 1315.7517 & 0.769686 & 1291.8516 & 0.76733 & 1157.4995 \\
\hline 0.67064 & 1301.2595 & 0.728761 & 1288.1531 & 0.721892 & 1152.5447 \\
\hline 0.622935 & 1288.4014 & 0.671556 & 1281.4229 & 0.667481 & 1148.2212 \\
\hline 0.574028 & 1275.2964 & 0.619638 & 1275.934 & 0.629863 & 1143.6779 \\
\hline 0.525499 & 1260.8765 & 0.569288 & 1270.2753 & 0.568956 & 1137.7734 \\
\hline 0.474328 & 1247.1973 & 0.516538 & 1263.2681 & 0.515758 & 1131.996 \\
\hline 0.421494 & 1232.3782 & 0.468545 & 1256.1656 & 0.479109 & 1129.0575 \\
\hline 0.37106 & 1218.0991 & 0.419594 & 1248.739 & 0.417314 & 1119.6521 \\
\hline 0.321594 & 1203.5607 & 0.369656 & 1239.3542 & 0.36706 & 1112.2667 \\
\hline 0.27106 & 1188.3894 & 0.323607 & 1229.9819 & 0.320861 & 1104.98 \\
\hline 0.221006 & 1171.739 & 0.271512 & 1218.4546 & 0.269002 & 1096.0868 \\
\hline 0.172678 & 1153.9443 & 0.223113 & 1206.1355 & 0.222099 & 1086.9113 \\
\hline 0.127166 & 1133.0337 & 0.178227 & 1192.2717 & 0.176454 & 1076.0946 \\
\hline 0.073587 & 1092.3856 & 0.128319 & 1171.5857 & 0.127784 & 1059.7125 \\
\hline 0.029515 & 878.3228 & & & 0.075636 & 1026.4409 \\
\hline 0.019722 & 804.1403 & & & & \\
\hline & & & & & \\
\hline
\end{tabular}

Table S31 cont.

\begin{tabular}{|l|l|}
\hline $\begin{array}{l}\text { Pristine } \\
(4756.530)\end{array}$ & \\
\hline $\mathrm{P} \mathrm{P}_{0}$ & $\begin{array}{l}\mathrm{N}_{2} \text { uptake } \\
\left(\mathrm{cc} \mathrm{g}^{-1}\right)\end{array}$ \\
\hline 0.001613 & 214.4736 \\
\hline 0.002102 & 274.8794 \\
\hline 0.002124 & 285.3504 \\
\hline 0.002182 & 296.9449 \\
\hline 0.002261 & 309.172 \\
\hline 0.002333 & 323.0916 \\
\hline 0.003269 & 504.1606 \\
\hline 0.004046 & 582.2675 \\
\hline
\end{tabular}




\begin{tabular}{|l|l|}
\hline 0.005213 & 630.1589 \\
\hline 0.010987 & 707.4757 \\
\hline 0.01543 & 740.5037 \\
\hline 0.020095 & 772.0238 \\
\hline 0.026863 & 817.5121 \\
\hline 0.031039 & 846.2365 \\
\hline 0.035258 & 876.2746 \\
\hline 0.040118 & 916.198 \\
\hline 0.046094 & 967.3579 \\
\hline 0.050386 & 994.4775 \\
\hline 0.056578 & 1019.2365 \\
\hline 0.061509 & 1030.8011 \\
\hline 0.067737 & 1041.3965 \\
\hline 0.073771 & 1049.5272 \\
\hline 0.080395 & 1056.8744 \\
\hline 0.087386 & 1063.6128 \\
\hline 0.094457 & 1069.5006 \\
\hline 0.10247 & 1075.4576 \\
\hline 0.108165 & 1079.3307 \\
\hline 0.159645 & 1106.6254 \\
\hline 0.213314 & 1128.1368 \\
\hline 0.268005 & 1147.1764 \\
\hline 0.319665 & 1164.0852 \\
\hline 0.370843 & 1180.1199 \\
\hline 0.42171 & 1195.7563 \\
\hline 0.471628 & 1212.6685 \\
\hline 0.524325 & 1227.8154 \\
\hline 0.574012 & 1243.1613 \\
\hline 0.625211 & 1259.3719 \\
\hline 0.678455 & 1275.345 \\
\hline 0.729632 & 1290.2459 \\
\hline 0.781299 & 1306.058 \\
\hline 0.831815 & 1314.2697 \\
\hline 0.88269 & 1346.1471 \\
\hline 0.92684 & 1368.8513 \\
\hline 0.973194 & 1400.595 \\
\hline 0.922844 & 1382.8718 \\
\hline 0.870291 & 1359.4294 \\
\hline 0.817638 & 1335.1436 \\
\hline 0.767245 & 1323.1193 \\
\hline 0.71758 & 1305.9381 \\
\hline 0.668519 & 1290.3993 \\
\hline 0.617673 & 1273.6865 \\
\hline & \\
\hline
\end{tabular}




\begin{tabular}{|l|l|}
\hline 0.568353 & 1258.0624 \\
\hline 0.522647 & 1245.4655 \\
\hline 0.474537 & 1198.2246 \\
\hline 0.420314 & 1178.3545 \\
\hline 0.369626 & 1164.4252 \\
\hline 0.31708 & 1149.4373 \\
\hline 0.268344 & 1134.7251 \\
\hline 0.216957 & 1117.2601 \\
\hline 0.168617 & 1100.2544 \\
\hline 0.121464 & 1079.6688 \\
\hline 0.075549 & 1046.4597 \\
\hline 0.028418 & 826.4085 \\
\hline 0.017546 & 751.7759 \\
\hline
\end{tabular}

Hexane resolvated samples

Table S32. $\mathrm{N}_{2}$ isotherm data for hexane resolvated FJI-1 samples used.

\begin{tabular}{|l|l|l|l|l|l|}
\hline $\begin{array}{l}\text { Hexane } \\
\text { resolvation 1 }\end{array}$ & $\begin{array}{l}\text { Hexane } \\
\text { resolvation 2 }\end{array}$ & & $\begin{array}{l}\text { Hexane } \\
\text { resolvation 3 }\end{array}$ & \\
\hline $\left.\mathrm{P}_{2} \mathrm{P}_{0} \mathrm{c} \mathrm{g}^{-1}\right)$ & $\mathrm{P} / \mathrm{P}_{0}$ & $\begin{array}{l}\mathrm{N}_{2} \text { uptake } \\
\left(\mathrm{cc} \mathrm{g}^{-1}\right)\end{array}$ & $\mathrm{P}^{-1} \mathrm{P}_{0}$ & $\begin{array}{l}\mathrm{N}_{2} \text { uptake } \\
\left(\mathrm{cc} \mathrm{g}^{-1}\right)\end{array}$ \\
\hline 0.000837 & 151.9327 & 0.000976 & 133.1055 & 0.001558 & 191.1914 \\
\hline 0.002152 & 288.6482 & 0.001576 & 183.9089 & 0.002063 & 233.7902 \\
\hline 0.002195 & 296.9886 & 0.002116 & 237.2547 & 0.002129 & 239.8164 \\
\hline 0.002274 & 306.0971 & 0.002123 & 241.9789 & 0.002211 & 246.6396 \\
\hline 0.002324 & 316.4015 & 0.00216 & 247.5004 & 0.002315 & 254.2434 \\
\hline 0.002388 & 327.2531 & 0.002204 & 253.5951 & 0.002389 & 262.3884 \\
\hline 0.002445 & 339.6888 & 0.002256 & 260.1445 & 0.003153 & 381.0689 \\
\hline 0.003168 & 501.3763 & 0.003092 & 400.8251 & 0.004073 & 531.1381 \\
\hline 0.004469 & 644.6486 & 0.004216 & 539.9586 & 0.005238 & 606.1764 \\
\hline 0.005298 & 677.2376 & 0.005015 & 580.2446 & 0.011404 & 699.767 \\
\hline 0.010804 & 759.1033 & 0.012405 & 674.9375 & 0.015707 & 731.6341 \\
\hline 0.016153 & 801.8651 & 0.01623 & 700.929 & 0.020322 & 761.6082 \\
\hline 0.020185 & 831.8626 & 0.020202 & 725.7217 & 0.025553 & 794.9451 \\
\hline 0.026006 & 874.7159 & 0.025646 & 759.4123 & 0.03068 & 827.4411 \\
\hline 0.030167 & 906.0178 & 0.031202 & 794.4332 & 0.035199 & 857.2173 \\
\hline 0.035909 & 951.8882 & 0.036077 & 827.1707 & 0.040712 & 897.5609 \\
\hline 0.040771 & 997.3436 & 0.040544 & 860.7187 & 0.04546 & 942.2631 \\
\hline 0.045784 & 1044.2948 & 0.045153 & 897.1861 & 0.05101 & 981.2085 \\
\hline 0.050739 & 1078.4296 & 0.05025 & 930.098 & 0.056315 & 1005.1951 \\
\hline 0.056609 & 1102.2096 & 0.056419 & 953.9139 & 0.061398 & 1020.1146 \\
\hline 0.061379 & 1114.2421 & 0.061479 & 966.3092 & 0.065115 & 1027.4429 \\
\hline 0.067321 & 1125.4177 & 0.065717 & 973.4398 & 0.070034 & 1034.9058 \\
\hline 0.072955 & 1133.7209 & 0.0702 & 979.2144 & 0.076133 & 1042.161 \\
\hline & & & & & \\
\hline
\end{tabular}




\begin{tabular}{|c|c|c|c|c|c|}
\hline 0.079691 & 1141.9907 & 0.076288 & 985.7825 & 0.081668 & 1046.3665 \\
\hline 0.085039 & 1147.7395 & 0.082509 & 991.2303 & 0.08793 & 1050.5354 \\
\hline 0.092311 & 1154.8121 & 0.087931 & 995.3005 & 0.094883 & 1055.2085 \\
\hline 0.100219 & 1161.7646 & 0.094278 & 999.8199 & 0.101865 & 1059.0513 \\
\hline 0.106805 & 1166.8368 & 0.099013 & 1002.6472 & 0.147095 & 1080.0779 \\
\hline 0.155256 & 1197.3275 & 0.151237 & 1025.0531 & 0.200264 & 1095.3242 \\
\hline 0.208061 & 1223.0037 & 0.196804 & 1037.8518 & 0.24755 & 1104.9076 \\
\hline 0.261331 & 1244.1584 & 0.247897 & 1048.4005 & 0.296134 & 1114.693 \\
\hline 0.313987 & 1263.3682 & 0.298554 & 1057.0729 & 0.348643 & 1123.098 \\
\hline 0.365591 & 1280.3474 & 0.345926 & 1064.1581 & 0.397019 & 1128.0938 \\
\hline 0.419705 & 1297.6909 & 0.395429 & 1071.5117 & 0.453475 & 1136.9414 \\
\hline 0.471931 & 1314.1328 & 0.448208 & 1078.6771 & 0.500761 & 1143.6168 \\
\hline 0.524743 & 1329.4392 & 0.495499 & 1083.7493 & 0.546688 & 1150.9734 \\
\hline 0.573366 & 1343.0721 & 0.545653 & 1088.4938 & 0.598782 & 1154.4835 \\
\hline 0.62492 & 1358.8084 & 0.599912 & 1094.3795 & 0.646023 & 1162.667 \\
\hline 0.674715 & 1373.7584 & 0.650087 & 1100.118 & 0.695735 & 1168.0396 \\
\hline 0.72426 & 1389.2225 & 0.700603 & 1104.4828 & 0.754179 & 1172.1301 \\
\hline 0.773347 & 1405.7621 & 0.750202 & 1108.8464 & 0.799499 & 1179.3962 \\
\hline 0.82257 & 1424.6473 & 0.798336 & 1113.9985 & 0.848491 & 1188.9095 \\
\hline 0.869605 & 1444.5802 & 0.846781 & 1119.1371 & 0.89824 & 1193.0045 \\
\hline 0.919086 & 1467.5315 & 0.902667 & 1122.574 & 0.947788 & 1210.6816 \\
\hline 0.962231 & 1509.3495 & 0.948996 & 1127.4613 & 0.927956 & 1208.4474 \\
\hline 0.922411 & 1485.1783 & 0.928284 & 1125.8282 & 0.8677 & 1199.1499 \\
\hline 0.87381 & 1455.1412 & 0.868299 & 1123.8513 & 0.813366 & 1190.7102 \\
\hline 0.821133 & 1428.5061 & 0.826638 & 1123.5189 & 0.769145 & 1188.88 \\
\hline 0.771037 & 1407.2159 & 0.765869 & 1118.5387 & 0.729005 & 1182.3428 \\
\hline 0.71771 & 1387.7776 & 0.728824 & 1115.7428 & 0.669477 & 1177.2045 \\
\hline 0.669753 & 1369.9359 & 0.666687 & 1112.2971 & 0.615098 & 1173.8385 \\
\hline 0.619035 & 1349.301 & 0.616903 & 1109.1649 & 0.566076 & 1168.1089 \\
\hline 0.57102 & 1328.5187 & 0.567815 & 1103.7993 & 0.527672 & 1163.9895 \\
\hline 0.521597 & 1308.776 & & & 0.471349 & 1153.3727 \\
\hline 0.47123 & 1282.928 & & & 0.416466 & 1145.4243 \\
\hline 0.418418 & 1264.2198 & & & 0.365092 & 1135.3431 \\
\hline 0.369016 & 1249.4172 & & & 0.329886 & 1131.0231 \\
\hline 0.320593 & 1233.9745 & & & 0.27127 & 1118.9985 \\
\hline 0.26871 & 1219.1473 & & & 0.22098 & 1107.5637 \\
\hline 0.217242 & 1204.2541 & & & 0.171283 & 1093.3538 \\
\hline \multirow[t]{4}{*}{0.167797} & 1185.5651 & & & 0.12563 & 1073.4066 \\
\hline & & & & 0.079724 & 1042.2501 \\
\hline & & & & 0.029144 & 817.3602 \\
\hline & & & & 0.018987 & 748.9588 \\
\hline
\end{tabular}




\section{$\mathrm{CH}_{2} \mathrm{Cl}_{2}$ resolvated samples}

Table S33. $\mathrm{N}_{2}$ isotherm data for $\mathrm{CH}_{2} \mathrm{Cl}_{2}$ resolvated $\mathrm{FJI}-1$ samples used.

\begin{tabular}{|c|c|c|c|c|c|}
\hline $\begin{array}{l}\mathrm{CH}_{2} \mathrm{Cl}_{2} \\
\text { resolvation } 1\end{array}$ & & $\begin{array}{l}\mathrm{CH}_{2} \mathrm{Cl}_{2} \\
\text { resolvation } 2\end{array}$ & & $\begin{array}{l}\mathrm{CH}_{2} \mathrm{Cl}_{2} \\
\text { resolvation } 3\end{array}$ & \\
\hline $\mathrm{P} / \mathrm{P}_{0}$ & $\begin{array}{l}\mathrm{N}_{2} \text { uptake } \\
\left(\mathrm{cc} \mathrm{g}^{-1}\right)\end{array}$ & $\mathrm{P} / \mathrm{P}_{0}$ & $\begin{array}{l}\mathrm{N}_{2} \text { uptake } \\
\left(\mathrm{cc} \mathrm{g}^{-1}\right)\end{array}$ & $\mathrm{P} / \mathrm{P}_{0}$ & $\begin{array}{l}\mathrm{N}_{2} \text { uptake } \\
\left(\mathrm{cc} \mathrm{g}^{-1}\right)\end{array}$ \\
\hline 0.002073 & 192.2444 & 0.001001 & 106.9938 & 0.000978 & 111.7791 \\
\hline 0.002051 & 195.878 & 0.002031 & 175.2183 & 0.001522 & 142.212 \\
\hline 0.00208 & 199.9054 & 0.002102 & 181.153 & 0.002037 & 175.6468 \\
\hline 0.002108 & 204.461 & 0.002167 & 187.7095 & 0.002081 & 178.7941 \\
\hline 0.002158 & 209.2806 & 0.002238 & 195.0235 & 0.002148 & 182.2087 \\
\hline 0.002208 & 214.853 & 0.00231 & 202.7706 & 0.002192 & 185.9917 \\
\hline 0.003041 & 323.6409 & 0.002395 & 211.5545 & 0.002236 & 190.1239 \\
\hline 0.004623 & 412.1578 & 0.003218 & 326.2233 & 0.003037 & 283.2949 \\
\hline 0.005029 & 421.8055 & 0.004262 & 405.9665 & 0.004155 & 427.0558 \\
\hline 0.012635 & 483.4973 & 0.005348 & 436.348 & 0.005479 & 485.8457 \\
\hline 0.0151 & 495.5254 & 0.010575 & 486.9402 & 0.010186 & 541.6765 \\
\hline 0.020064 & 517.2411 & 0.016274 & 516.7559 & 0.016636 & 578.9854 \\
\hline 0.025905 & 542.6412 & 0.020572 & 537.3584 & 0.02007 & 596.4547 \\
\hline 0.030349 & 562.0692 & 0.026757 & 566.8781 & 0.025424 & 623.321 \\
\hline 0.035777 & 587.6574 & 0.030475 & 585.2203 & 0.030521 & 649.6567 \\
\hline 0.040756 & 614.2443 & 0.036259 & 614.9669 & 0.035125 & 674.0743 \\
\hline 0.045342 & 639.1647 & 0.040464 & 640.3153 & 0.041361 & 712.1793 \\
\hline 0.050663 & 662.8555 & 0.045584 & 671.2219 & 0.046465 & 745.0123 \\
\hline 0.056261 & 678.0465 & 0.05001 & 691.6805 & 0.050702 & 766.5646 \\
\hline 0.060977 & 685.8995 & 0.055794 & 707.9177 & 0.055931 & 783.4675 \\
\hline 0.065585 & 691.4324 & 0.060542 & 716.2512 & 0.060512 & 792.8899 \\
\hline 0.070485 & 696.2957 & 0.066527 & 723.8746 & 0.066256 & 800.9733 \\
\hline 0.076675 & 701.3066 & 0.072254 & 729.6578 & 0.071493 & 806.614 \\
\hline 0.083099 & 705.6993 & 0.079083 & 735.3177 & 0.07506 & 809.7383 \\
\hline 0.089802 & 709.6871 & 0.086126 & 740.5502 & 0.080737 & 813.9717 \\
\hline 0.097743 & 713.817 & 0.093169 & 745.2451 & 0.085797 & 817.2532 \\
\hline 0.103385 & 716.5191 & 0.098561 & 748.4833 & 0.092137 & 820.9806 \\
\hline 0.148278 & 732.1229 & 0.105975 & 752.5082 & 0.098822 & 824.2389 \\
\hline 0.197488 & 745.0589 & 0.154112 & 773.5446 & 0.14892 & 840.5583 \\
\hline 0.249968 & 756.56 & 0.20741 & 789.8936 & 0.198335 & 850.1262 \\
\hline 0.302924 & 767.1202 & 0.261246 & 806.2961 & 0.249037 & 857.6395 \\
\hline 0.354471 & 776.8522 & 0.315316 & 820.2479 & 0.301033 & 863.3267 \\
\hline 0.405711 & 786.2768 & 0.366084 & 832.9313 & 0.35083 & 868.1129 \\
\hline 0.45683 & 795.7105 & 0.417352 & 845.1272 & 0.397001 & 872.1378 \\
\hline 0.509686 & 804.929 & 0.470515 & 855.3375 & 0.446894 & 876.3922 \\
\hline 0.55869 & 813.2269 & 0.521061 & 865.2678 & 0.497242 & 879.6066 \\
\hline 0.609467 & 822.4908 & 0.572322 & 876.4006 & 0.545811 & 883.5632 \\
\hline
\end{tabular}




\begin{tabular}{|l|l|l|l|l|l|}
\hline 0.662352 & 831.6868 & 0.624963 & 887.1 & 0.598454 & 886.7615 \\
\hline 0.714105 & 840.7343 & 0.67641 & 898.7399 & 0.646097 & 890.4675 \\
\hline 0.763322 & 850.8333 & 0.727978 & 910.2678 & 0.695489 & 894.094 \\
\hline 0.814007 & 859.9349 & 0.776264 & 922.6285 & 0.746992 & 896.8578 \\
\hline 0.866842 & 868.7599 & 0.825337 & 934.4485 & 0.798165 & 900.4993 \\
\hline 0.915895 & 877.1062 & 0.876927 & 949.1701 & 0.845094 & 904.041 \\
\hline 0.961444 & 887.7598 & 0.923791 & 965.2716 & 0.901826 & 906.8977 \\
\hline 0.919727 & 878.2084 & 0.964305 & 996.4536 & 0.952653 & 910.911 \\
\hline 0.870069 & 869.4971 & 0.920988 & 983.4266 & 0.929059 & 909.76 \\
\hline 0.821842 & 861.7629 & 0.873223 & 968.4198 & 0.864965 & 906.7061 \\
\hline 0.772795 & 853.2968 & 0.818623 & 951.0449 & 0.827965 & 905.1765 \\
\hline 0.722787 & 844.1483 & 0.769243 & 938.1302 & 0.763849 & 901.002 \\
\hline 0.672644 & 836.147 & 0.719769 & 924.9738 & 0.725819 & 898.9051 \\
\hline 0.622708 & 828.0434 & 0.670968 & 911.9127 & 0.664858 & 895.5468 \\
\hline 0.576169 & 820.6992 & 0.617991 & 896.3981 & 0.623599 & 892.0252 \\
\hline 0.526838 & 812.611 & 0.571442 & 882.9533 & 0.568411 & 888.7578 \\
\hline 0.474266 & 803.9286 & 0.523056 & 868.5564 & 0.516768 & 884.8063 \\
\hline 0.425084 & 795.5359 & 0.472975 & 835.4877 & 0.469303 & 881.4809 \\
\hline 0.373345 & 786.5641 & 0.421035 & 824.0798 & 0.419734 & 877.7969 \\
\hline 0.323509 & 777.3699 & 0.369674 & 812.6953 & 0.36737 & 872.9348 \\
\hline 0.275908 & 768.5219 & 0.317805 & 800.1506 & 0.321287 & 868.5917 \\
\hline 0.226356 & 757.8551 & 0.26678 & 789.697 & 0.271402 & 862.6356 \\
\hline 0.176135 & 745.9007 & 0.21687 & 778.529 & 0.222252 & 855.9057 \\
\hline 0.129681 & 732.3221 & 0.168462 & 766.2231 & 0.171535 & 846.7725 \\
\hline 0.074901 & 706.317 & & & & \\
\hline 0.028319 & 562.8373 & & & & \\
\hline 0.019523 & 523.1446 & & & & \\
\hline & & & & & \\
\hline
\end{tabular}

\section{THF resolvated samples}

Table S34. $\mathrm{N}_{2}$ isotherm data for THF resolvated FJI-1 samples used.

\begin{tabular}{|l|l|l|l|l|l|}
\hline $\begin{array}{l}\text { THF } \\
\text { resolvation 1 }\end{array}$ & $\begin{array}{l}\text { THF } \\
\text { resolvation 2 }\end{array}$ & $\begin{array}{l}\text { THF } \\
\text { resolvation 3 }\end{array}$ & \\
\hline $\mathrm{P}_{\mathrm{P}}$ & $\begin{array}{l}\mathrm{N}_{2} \text { uptake } \\
\left(\mathrm{cc} \mathrm{g}^{-1}\right)\end{array}$ & $\mathrm{P} / \mathrm{P}_{0}$ & $\begin{array}{l}\mathrm{N}_{2} \text { uptake } \\
\left(\mathrm{cc} \mathrm{g}^{-1}\right)\end{array}$ & $\mathrm{P} / \mathrm{P}_{0}$ & $\begin{array}{l}\mathrm{N}_{2} \text { uptake } \\
\left(\mathrm{cc} \mathrm{g}^{-1}\right)\end{array}$ \\
\hline 0.001535 & 99.4 & 0.001187 & 66.4379 & 0.000853 & 77.7864 \\
\hline 0.002181 & 123.6515 & 0.002074 & 99.1526 & 0.001743 & 117.0078 \\
\hline 0.002321 & 128.9942 & 0.002195 & 104.6877 & 0.002029 & 130.5963 \\
\hline 0.002461 & 135.1701 & 0.002317 & 110.7663 & 0.00211 & 134.2426 \\
\hline 0.002571 & 142.0449 & 0.002417 & 117.5935 & 0.002198 & 138.435 \\
\hline 0.002681 & 149.5272 & 0.002517 & 124.8154 & 0.002279 & 143.0039 \\
\hline 0.003048 & 178.7138 & 0.002646 & 132.9753 & 0.002353 & 148.1006 \\
\hline 0.004106 & 254.2664 & 0.003032 & 163.2031 & 0.003162 & 218.8742 \\
\hline 0.005119 & 282.5518 & 0.005027 & 229.7147 & 0.004096 & 298.7592 \\
\hline
\end{tabular}




\begin{tabular}{|c|c|c|c|c|c|}
\hline 0.011766 & 328.3297 & 0.005742 & 237.2635 & 0.005022 & 329.8499 \\
\hline 0.016092 & 342.7237 & 0.011727 & 265.6517 & 0.013838 & 393.6106 \\
\hline 0.021557 & 358.6699 & 0.015059 & 275.6288 & 0.015412 & 399.7972 \\
\hline 0.026368 & 372.4202 & 0.021287 & 292.427 & 0.021051 & 419.996 \\
\hline 0.031135 & 386.269 & 0.026585 & 306.5981 & 0.026316 & 438.5206 \\
\hline 0.036004 & 400.5461 & 0.030167 & 316.3686 & 0.030095 & 451.8682 \\
\hline 0.040367 & 414.976 & 0.036059 & 333.1559 & 0.035029 & 469.8148 \\
\hline 0.046566 & 436.9253 & 0.041 & 349.4403 & 0.041102 & 495.0338 \\
\hline 0.051472 & 450.8665 & 0.046005 & 365.642 & 0.045463 & 514.2061 \\
\hline 0.055248 & 458.3276 & 0.05219 & 379.5676 & 0.050735 & 533.1323 \\
\hline 0.060382 & 465.4396 & 0.058054 & 387.2566 & 0.056477 & 545.9399 \\
\hline 0.066625 & 471.0816 & 0.063981 & 392.5247 & 0.062852 & 554.3821 \\
\hline 0.074153 & 475.8947 & 0.070982 & 397.3347 & 0.06697 & 558.3603 \\
\hline 0.079823 & 478.7985 & 0.077088 & 400.7634 & 0.071411 & 561.8886 \\
\hline 0.084575 & 480.9607 & 0.082958 & 403.7506 & 0.076087 & 564.8985 \\
\hline 0.091494 & 483.5896 & 0.089458 & 406.8423 & 0.08269 & 568.6577 \\
\hline 0.098259 & 485.8234 & 0.095457 & 409.6029 & 0.087433 & 570.9273 \\
\hline 0.102291 & 487.1512 & 0.10097 & 411.8961 & 0.094374 & 574.0942 \\
\hline 0.148005 & 496.7677 & 0.107191 & 414.3137 & 0.100249 & 576.3259 \\
\hline 0.197075 & 503.3286 & 0.158338 & 431.8017 & 0.146998 & 589.0316 \\
\hline 0.248636 & 508.5381 & 0.210542 & 446.664 & 0.195696 & 598.153 \\
\hline 0.299844 & 512.6049 & 0.264435 & 461.6436 & 0.249754 & 605.5605 \\
\hline 0.348679 & 515.8542 & 0.317776 & 474.6356 & 0.296003 & 611.7783 \\
\hline 0.402046 & 518.752 & 0.369874 & 487.0565 & 0.349083 & 616.9043 \\
\hline 0.451638 & 520.9014 & 0.419347 & 498.139 & 0.396649 & 622.5385 \\
\hline 0.501282 & 523.5961 & 0.470093 & 509.9836 & 0.446332 & 627.7256 \\
\hline 0.551608 & 526.2691 & 0.521812 & 522.3054 & 0.501066 & 632.8038 \\
\hline 0.600143 & 528.3829 & 0.570642 & 535.2892 & 0.546529 & 636.8782 \\
\hline 0.649838 & 528.4185 & 0.620244 & 546.4153 & 0.597139 & 640.5432 \\
\hline 0.703043 & 529.485 & 0.674922 & 557.2164 & 0.651572 & 645.4377 \\
\hline 0.753421 & 529.6149 & 0.726369 & 568.2493 & 0.699497 & 651.3873 \\
\hline 0.805437 & 531.3127 & 0.777487 & 580.3022 & 0.748629 & 656.1694 \\
\hline 0.855315 & 530.1478 & 0.825838 & 591.8029 & 0.79918 & 660.1942 \\
\hline 0.903791 & 530.6296 & 0.876184 & 603.2642 & 0.847672 & 664.5084 \\
\hline 0.952112 & 531.2421 & 0.928124 & 616.8057 & 0.898083 & 670.7524 \\
\hline 0.928815 & 530.8903 & 0.973614 & 633.9718 & 0.948531 & 682.4633 \\
\hline 0.863145 & 534.9543 & 0.921967 & 617.4456 & 0.925318 & 680.0511 \\
\hline 0.825172 & 536.2861 & 0.871908 & 605.2487 & 0.875642 & 674.0383 \\
\hline 0.76673 & 536.3122 & 0.819495 & 591.7262 & 0.828819 & 670.809 \\
\hline 0.727465 & 537.8343 & 0.770086 & 579.4321 & 0.766166 & 666.3308 \\
\hline 0.679408 & 536.6839 & 0.718304 & 565.9728 & 0.728843 & 663.7526 \\
\hline 0.618424 & 536.2325 & 0.670532 & 553.607 & 0.666263 & 660.1935 \\
\hline 0.567201 & 535.498 & 0.619014 & 539.9462 & & \\
\hline
\end{tabular}




\begin{tabular}{|l|l|l|l|l|l|}
\hline 0.516221 & 533.2805 & 0.57052 & 526.2281 & & \\
\hline 0.477646 & 531.0718 & 0.522584 & 511.3804 & & \\
\hline 0.416398 & 527.8442 & 0.473146 & 495.0053 & & \\
\hline 0.364331 & 524.8655 & 0.422407 & 478.1979 & & \\
\hline 0.313997 & 521.35 & 0.369573 & 465.8208 & & \\
\hline 0.265771 & 516.9645 & 0.317404 & 453.9284 & & \\
\hline 0.217053 & 511.9765 & 0.265807 & 444.0652 & & \\
\hline 0.171545 & 505.7096 & 0.216556 & 433.3089 & & \\
\hline 0.119052 & 495.3866 & 0.167376 & 422.0198 & & \\
\hline 0.072427 & 478.0843 & & & & \\
\hline 0.029541 & 384.1942 & & & & \\
\hline 0.018494 & 351.2871 & & & & \\
\hline
\end{tabular}

DMF resolvated samples

Table S35. $\mathrm{N}_{2}$ isotherm data for DMF resolvated FJI-1 samples used.

\begin{tabular}{|l|l|l|l|l|l|}
\hline $\begin{array}{l}\text { DMF } \\
\text { resolvation 1 }\end{array}$ & $\begin{array}{l}\text { DMF } \\
\text { resolvation 2 }\end{array}$ & & $\begin{array}{l}\text { DMF } \\
\text { resolvation 3 }\end{array}$ & \\
\hline $\mathrm{P}_{0} \mathrm{P}_{0}$ & $\begin{array}{l}\mathrm{N}_{2} \text { uptake } \\
\left(\mathrm{cc} \mathrm{g}^{-1}\right)\end{array}$ & $\mathrm{P}_{0}$ & $\begin{array}{l}\mathrm{N}_{2} \text { uptake } \\
\left(\mathrm{cc} \mathrm{g}^{-1}\right)\end{array}$ & $\mathrm{P} \mathrm{P}_{0}$ & $\begin{array}{l}\mathrm{N}_{2} \text { uptake } \\
\left(\mathrm{cc} \mathrm{g}^{-1}\right)\end{array}$ \\
\hline 0.001843 & 105.5009 & 0.002182 & 86.877 & 0.001634 & 122.1731 \\
\hline 0.002095 & 118.2418 & 0.002277 & 90.5662 & 0.002146 & 150.1643 \\
\hline 0.002124 & 121.0206 & 0.00235 & 94.696 & 0.002174 & 154.9531 \\
\hline 0.002196 & 124.1723 & 0.002467 & 99.3932 & 0.002239 & 160.2841 \\
\hline 0.002268 & 127.5195 & 0.002576 & 104.6533 & 0.002318 & 166.2816 \\
\hline 0.002333 & 131.0926 & 0.002693 & 110.3316 & 0.002405 & 172.6652 \\
\hline 0.003017 & 164.537 & 0.003095 & 132.9367 & 0.003067 & 226.0465 \\
\hline 0.004262 & 197.5685 & 0.004496 & 190.5133 & 0.004565 & 283.8396 \\
\hline 0.005522 & 210.2387 & 0.005189 & 201.8112 & 0.005321 & 295.2847 \\
\hline 0.011008 & 231.9419 & 0.01248 & 236.7818 & 0.011174 & 331.9411 \\
\hline 0.016494 & 244.7543 & 0.015093 & 243.9116 & 0.015394 & 347.1534 \\
\hline 0.020756 & 253.5825 & 0.020705 & 256.8804 & 0.022003 & 368.032 \\
\hline 0.025191 & 262.3623 & 0.02677 & 270.0746 & 0.025978 & 380.4869 \\
\hline 0.031635 & 274.4598 & 0.031617 & 280.7146 & 0.030298 & 393.5916 \\
\hline 0.035587 & 282.2498 & 0.03666 & 291.9409 & 0.03528 & 408.9428 \\
\hline 0.040987 & 294.0439 & 0.041061 & 302.9602 & 0.04014 & 425.7575 \\
\hline 0.045083 & 302.8046 & 0.045476 & 314.3448 & 0.046447 & 447.9984 \\
\hline 0.051743 & 313.2715 & 0.050819 & 325.6587 & 0.050746 & 459.6217 \\
\hline 0.056106 & 317.8363 & 0.055453 & 332.2785 & 0.055469 & 467.9309 \\
\hline 0.06098 & 321.4524 & 0.060825 & 337.4977 & 0.060653 & 474.2263 \\
\hline 0.066675 & 324.6858 & 0.067641 & 342.1564 & 0.066881 & 479.7084 \\
\hline 0.072384 & 327.273 & 0.072925 & 345.0182 & 0.073094 & 484.0906 \\
\hline 0.078684 & 329.6148 & 0.079596 & 347.9852 & 0.080237 & 488.2573 \\
\hline 0.085415 & 331.8643 & 0.085106 & 350.0965 & 0.086018 & 491.2665 \\
\hline & & & & & \\
\hline
\end{tabular}




\begin{tabular}{|c|c|c|c|c|c|}
\hline 0.092989 & 334.144 & 0.091835 & 352.492 & 0.09324 & 494.5093 \\
\hline 0.100037 & 335.9786 & 0.096601 & 354.1372 & 0.101462 & 497.8228 \\
\hline 0.106596 & 337.6137 & 0.101674 & 355.748 & 0.107553 & 500.085 \\
\hline 0.157158 & 346.2179 & 0.151259 & 365.8076 & 0.158623 & 513.8784 \\
\hline 0.208765 & 352.9464 & 0.198924 & 372.5741 & 0.213998 & 524.882 \\
\hline 0.263481 & 358.9941 & 0.251071 & 378.624 & 0.26874 & 534.1788 \\
\hline 0.31495 & 364.1947 & 0.299335 & 383.6443 & 0.321904 & 541.9192 \\
\hline 0.366153 & 369.2328 & 0.349344 & 388.3457 & 0.37378 & 549.697 \\
\hline 0.418818 & 374.0665 & 0.397265 & 392.7112 & 0.42377 & 556.4325 \\
\hline 0.472634 & 379.1476 & 0.44747 & 397.2994 & 0.474825 & 563.657 \\
\hline 0.525695 & 383.5604 & 0.496136 & 400.777 & 0.52647 & 570.4149 \\
\hline 0.577013 & 388.3978 & 0.545436 & 404.3236 & 0.579477 & 577.2065 \\
\hline 0.625862 & 392.7725 & 0.597642 & 407.813 & 0.630661 & 583.8881 \\
\hline 0.674458 & 396.8519 & 0.65092 & 412.7683 & 0.68186 & 590.4355 \\
\hline 0.726223 & 401.1845 & 0.698483 & 414.9958 & 0.733549 & 596.6363 \\
\hline 0.777764 & 405.7158 & 0.74609 & 418.8673 & 0.783114 & 602.884 \\
\hline 0.827815 & 410.1487 & 0.797026 & 422.3629 & 0.831793 & 608.5571 \\
\hline 0.876787 & 414.7934 & 0.852464 & 428.3757 & 0.881696 & 615.6213 \\
\hline 0.926154 & 419.7018 & 0.905895 & 431.0733 & 0.93152 & 624.1067 \\
\hline 0.973814 & 426.4112 & 0.950291 & 437.1119 & 0.979083 & 635.829 \\
\hline 0.922885 & 420.2292 & 0.929126 & 435.3372 & 0.925638 & 622.4951 \\
\hline 0.868853 & 414.8594 & 0.86409 & 433.7423 & 0.86937 & 612.3805 \\
\hline 0.821084 & 409.8422 & 0.825686 & 431.7118 & 0.817544 & 605.3024 \\
\hline 0.768499 & 404.6545 & 0.766219 & 428.0843 & 0.767706 & 598.4019 \\
\hline 0.720391 & 400.1205 & 0.728231 & 427.1409 & 0.717169 & 591.2368 \\
\hline 0.667468 & 395.551 & 0.667874 & 425.8639 & 0.667136 & 584.1506 \\
\hline 0.620289 & 391.4812 & 0.61769 & 421.4505 & 0.617248 & 576.6991 \\
\hline 0.571707 & 387.2426 & 0.578695 & 419.3212 & 0.568043 & 570.361 \\
\hline 0.522239 & 382.642 & 0.517936 & 414.9362 & 0.522294 & 565.0627 \\
\hline 0.469654 & 378.4847 & 0.467169 & 412.1688 & 0.472218 & 557.8211 \\
\hline 0.417234 & 373.7252 & 0.421955 & 408.0376 & 0.419867 & 551.032 \\
\hline 0.367615 & 369.6366 & 0.37191 & 403.9538 & 0.369431 & 545.2798 \\
\hline 0.316153 & 365.0395 & 0.321537 & 398.4438 & 0.317519 & 538.4686 \\
\hline 0.265641 & 360.5829 & 0.265026 & 392.5736 & 0.270352 & 532.0743 \\
\hline 0.218433 & 355.9782 & 0.21755 & 386.083 & 0.217195 & 523.7164 \\
\hline 0.171032 & 350.7755 & 0.172169 & 379.133 & 0.168473 & 515.4057 \\
\hline 0.124804 & 344.2573 & 0.120781 & 368.5778 & 0.121709 & 505.1357 \\
\hline 0.077582 & 333.5765 & 0.074348 & 353.8803 & 0.076226 & 488.3389 \\
\hline 0.027315 & 273.5468 & 0.028018 & 282.3004 & 0.026129 & 386.5906 \\
\hline 0.019201 & 258.1363 & 0.017334 & 257.7118 & 0.017806 & 360.96 \\
\hline
\end{tabular}




\section{DMSO resolvated samples}

Table S36. $\mathrm{N}_{2}$ isotherm data for DMSO resolvated FJI-1 samples used.

\begin{tabular}{|c|c|c|c|c|c|}
\hline $\begin{array}{l}\text { DMSO } \\
\text { resolvation } 1\end{array}$ & & $\begin{array}{l}\text { DMSO } \\
\text { resolvation } 2\end{array}$ & & $\begin{array}{l}\text { DMSO } \\
\text { resolvation } 3\end{array}$ & \\
\hline $\mathrm{P} / \mathrm{P}_{0}$ & $\begin{array}{l}\mathrm{N}_{2} \text { uptake } \\
\left(\mathrm{cc} \mathrm{g} \mathrm{g}^{-1}\right)\end{array}$ & $\mathrm{P} / \mathrm{P}_{0}$ & $\begin{array}{l}N_{2} \text { uptake } \\
\left(\mathrm{cc} \mathrm{g}^{-1}\right)\end{array}$ & $\mathrm{P} / \mathrm{P}_{0}$ & $\begin{array}{l}N_{2} \text { uptake } \\
\left(\mathrm{cc} \mathrm{g}^{-1}\right)\end{array}$ \\
\hline 0.001194 & 182.7782 & 0.001494 & 101.8151 & 0.001593 & 182.182 \\
\hline 0.002231 & 271.0053 & 0.001657 & 106.5779 & 0.002048 & 212.0695 \\
\hline 0.00226 & 276.2863 & 0.002034 & 120.3136 & 0.002172 & 219.9595 \\
\hline 0.00231 & 282.1266 & 0.002205 & 126.2344 & 0.002304 & 228.9807 \\
\hline 0.002367 & 288.6637 & 0.002375 & 132.9979 & 0.002429 & 238.9566 \\
\hline 0.00241 & 295.6199 & 0.002523 & 140.4455 & 0.002561 & 249.8512 \\
\hline 0.002474 & 303.5332 & 0.002678 & 148.2294 & 0.003053 & 307.2515 \\
\hline 0.003017 & 369.8882 & 0.003018 & 167.8419 & 0.004125 & 416.3678 \\
\hline 0.004562 & 471.655 & 0.004024 & 217.4802 & 0.005548 & 473.6187 \\
\hline 0.005113 & 485.9919 & 0.005815 & 247.4341 & 0.010979 & 537.6948 \\
\hline 0.011848 & 556.9438 & 0.011474 & 277.6571 & 0.015243 & 564.6497 \\
\hline 0.016381 & 584.4628 & 0.016113 & 292.1469 & 0.0203 & 590.9799 \\
\hline 0.02143 & 611.6127 & 0.021469 & 306.7794 & 0.025445 & 616.1368 \\
\hline 0.025241 & 631.4401 & 0.02502 & 316.0127 & 0.031441 & 645.0292 \\
\hline 0.030568 & 658.7178 & 0.031078 & 331.1965 & 0.03569 & 666.4329 \\
\hline 0.036052 & 686.6638 & 0.03696 & 345.8659 & 0.040138 & 688.7286 \\
\hline 0.040285 & 710.1234 & 0.040318 & 354.7939 & 0.046126 & 720.7272 \\
\hline 0.045848 & 739.2612 & 0.046141 & 370.5483 & 0.050537 & 740.0429 \\
\hline 0.051483 & 760.3092 & 0.050283 & 379.7928 & 0.057575 & 760.5698 \\
\hline 0.055937 & 770.564 & 0.055795 & 388.4689 & 0.061671 & 768.3044 \\
\hline 0.061543 & 779.7903 & 0.061691 & 394.588 & 0.067953 & 776.6087 \\
\hline 0.067013 & 786.5272 & 0.068771 & 400.0138 & 0.073281 & 782.101 \\
\hline 0.072662 & 792.2086 & 0.073572 & 403.075 & 0.079967 & 787.8111 \\
\hline 0.079355 & 797.8935 & 0.080481 & 406.7052 & 0.086404 & 792.5243 \\
\hline 0.084446 & 801.8207 & 0.086851 & 410.1313 & 0.090403 & 795.0911 \\
\hline 0.091746 & 806.9634 & 0.09166 & 412.3065 & 0.097288 & 799.282 \\
\hline 0.097688 & 810.5867 & 0.098044 & 415.3505 & 0.102528 & 802.1899 \\
\hline 0.104388 & 814.3807 & 0.104295 & 417.737 & 0.148852 & 821.5743 \\
\hline 0.153196 & 836.182 & 0.146182 & 431.1873 & 0.200336 & 836.5777 \\
\hline 0.207825 & 853.8012 & 0.200912 & 444.035 & 0.248562 & 848.1207 \\
\hline 0.260237 & 867.291 & 0.245743 & 453.3145 & 0.298512 & 857.9769 \\
\hline 0.31561 & 878.905 & 0.298216 & 461.9812 & 0.348388 & 865.6654 \\
\hline 0.369037 & 889.1745 & 0.348225 & 469.8575 & 0.397972 & 873.7139 \\
\hline 0.419504 & 897.7117 & 0.396571 & 476.8926 & 0.447085 & 881.154 \\
\hline 0.470351 & 906.2589 & 0.451884 & 484.6936 & 0.499288 & 888.3274 \\
\hline 0.522241 & 913.7651 & 0.496723 & 489.1829 & 0.54733 & 894.4666 \\
\hline
\end{tabular}




\begin{tabular}{|l|l|l|l|l|l|}
\hline 0.576233 & 922.8646 & 0.552932 & 495.2327 & 0.59739 & 897.5685 \\
\hline 0.627458 & 931.3682 & 0.597341 & 499.9036 & 0.646952 & 903.9473 \\
\hline 0.678018 & 939.2852 & 0.646737 & 504.8536 & 0.697122 & 910.9775 \\
\hline 0.729544 & 948.0156 & 0.696362 & 510.514 & 0.748958 & 915.1849 \\
\hline 0.778853 & 956.8928 & 0.74666 & 516.1556 & 0.799026 & 920.2993 \\
\hline 0.827511 & 966.7899 & 0.796078 & 521.1843 & 0.848308 & 927.189 \\
\hline 0.876019 & 977.7419 & 0.854063 & 528.4504 & 0.895506 & 935.8611 \\
\hline 0.92565 & 991.5732 & 0.89879 & 534.8665 & 0.951063 & 945.5508 \\
\hline 0.96951 & 1012.8243 & 0.945737 & 544.8519 & 0.926352 & 942.8245 \\
\hline 0.928424 & 998.8966 & 0.927494 & 541.9094 & 0.86714 & 938.0795 \\
\hline 0.872458 & 985.6812 & 0.86857 & 533.88 & 0.826812 & 936.2086 \\
\hline 0.820732 & 974.4836 & 0.827356 & 530.8848 & 0.76448 & 933.389 \\
\hline 0.770129 & 964.5755 & 0.774498 & 523.8409 & 0.724504 & 931.0665 \\
\hline 0.718268 & 953.9378 & 0.722647 & 516.8517 & 0.666393 & 922.948 \\
\hline 0.670704 & 943.7743 & 0.6643 & 513.4155 & 0.615408 & 917.8532 \\
\hline 0.619507 & 931.9305 & 0.623182 & 507.0351 & 0.574287 & 914.9234 \\
\hline 0.570877 & 920.2847 & 0.568801 & 500.7508 & 0.518436 & 909.2845 \\
\hline 0.52302 & 908.8976 & 0.518525 & 496.652 & 0.469741 & 897.2615 \\
\hline 0.47537 & 893.6193 & 0.470616 & 486.7854 & 0.418037 & 887.0951 \\
\hline 0.423795 & 878.1179 & 0.419393 & 476.7433 & 0.365907 & 878.468 \\
\hline 0.36943 & 868.9553 & 0.369428 & 469.004 & 0.314496 & 867.7056 \\
\hline 0.318541 & 860.679 & 0.314928 & 459.9644 & 0.265141 & 857.2428 \\
\hline 0.266387 & 852.5112 & 0.267411 & 452.5316 & 0.218875 & 846.1921 \\
\hline 0.221146 & 843.4249 & 0.219022 & 443.5633 & 0.172741 & 832.6379 \\
\hline & & 0.173754 & 433.607 & 0.122321 & 812.7501 \\
\hline & & 0.12316 & 419.9548 & 0.074507 & 781.9907 \\
\hline & & 0.073986 & 400.5178 & 0.027103 & 621.5972 \\
\hline & & 0.029059 & 324.1093 & 0.017827 & 573.0786 \\
\hline
\end{tabular}

\section{$\mathrm{CH}_{2} \mathrm{Cl}_{2} \mathrm{RSE}$ samples}

Table S37. $\mathrm{N}_{2}$ isotherm data for $\mathrm{CH}_{2} \mathrm{Cl}_{2}$ RSE FJI-1 samples used.

\begin{tabular}{|l|l|l|l|l|l|}
\hline $\mathrm{CH}_{2} \mathrm{Cl}_{2} \mathrm{RSE} 1$ & & $\mathrm{CH}_{2} \mathrm{Cl}_{2} \mathrm{RSE} 2$ & & $\mathrm{CH}_{2} \mathrm{Cl}_{2} \mathrm{RSE} 3$ & \\
\hline $\mathrm{P} / \mathrm{P}_{0}$ & $\begin{array}{l}\mathrm{N}_{2} \text { uptake } \\
(\mathrm{cc} \mathrm{g})\end{array}$ & $\mathrm{P} / \mathrm{P}_{0}$ & $\begin{array}{l}\mathrm{N}_{2} \text { uptake } \\
\left.(\mathrm{cc} \mathrm{g})^{-1}\right)\end{array}$ & $\mathrm{P} / \mathrm{P}_{0}$ & $\begin{array}{l}\mathrm{N}_{2} \text { uptake } \\
\left(\mathrm{cc} \mathrm{g}^{-1}\right)\end{array}$ \\
\hline 0.000869 & 144.9106 & 0.001762 & 201.2521 & 0.000905 & 121.0356 \\
\hline 0.001671 & 213.1819 & 0.002052 & 232.5175 & 0.001589 & 172.8371 \\
\hline 0.002157 & 257.1107 & 0.00211 & 240.4373 & 0.002096 & 211.5266 \\
\hline 0.002216 & 261.0222 & 0.002175 & 249.4153 & 0.002118 & 215.1526 \\
\hline 0.002261 & 265.466 & 0.00224 & 258.9105 & 0.002148 & 219.1605 \\
\hline 0.002312 & 270.2891 & 0.002313 & 269.6948 & 0.002206 & 223.5986 \\
\hline 0.002319 & 275.5709 & 0.00303 & 399.4659 & 0.002265 & 228.3745 \\
\hline 0.003048 & 394.6068 & 0.004125 & 526.8072 & 0.003037 & 336.0391 \\
\hline 0.004087 & 585.9756 & 0.005466 & 576.7866 & 0.004045 & 504.5173 \\
\hline
\end{tabular}




\begin{tabular}{|c|c|c|c|c|c|}
\hline 0.005324 & 662.2448 & 0.011339 & 644.4868 & 0.005244 & 572.9031 \\
\hline 0.011258 & 755.5151 & 0.01508 & 669.588 & 0.011377 & 656.287 \\
\hline 0.015595 & 789.3096 & 0.021068 & 706.2518 & 0.015694 & 685.6537 \\
\hline 0.020404 & 822.8378 & 0.025056 & 730.5659 & 0.020482 & 714.5815 \\
\hline 0.025212 & 855.9459 & 0.030972 & 767.0045 & 0.025579 & 745.6003 \\
\hline 0.030042 & 889.8796 & 0.035452 & 796.1495 & 0.030595 & 776.1283 \\
\hline 0.036698 & 938.5084 & 0.040636 & 834.1822 & 0.035044 & 804.5115 \\
\hline 0.040446 & 970.2141 & 0.04574 & 873.4727 & 0.04117 & 849.0341 \\
\hline 0.045954 & 1018.7546 & 0.051388 & 905.4422 & 0.045804 & 885.4125 \\
\hline 0.051211 & 1056.4351 & 0.055296 & 919.1713 & 0.0514 & 919.1725 \\
\hline 0.056005 & 1078.5371 & 0.062582 & 935.893 & 0.055916 & 936.4054 \\
\hline 0.061866 & 1094.9707 & 0.0677 & 943.5919 & 0.060505 & 947.2994 \\
\hline 0.067042 & 1104.7163 & 0.073268 & 950.4177 & 0.066168 & 956.7775 \\
\hline 0.070473 & 1109.8087 & 0.079482 & 956.6752 & 0.070044 & 961.9503 \\
\hline 0.076924 & 1117.9965 & 0.08568 & 962.0739 & 0.07 & 969.1145 \\
\hline 0.081761 & 1122.9998 & 0.09243 & 967.2875 & 0.082039 & 973.5953 \\
\hline 0.085877 & 1126.9545 & 0.09844 & 971.5121 & 0.086525 & 977.0651 \\
\hline 0.092048 & 1131.9899 & 0.104037 & 975.0795 & 0.091747 & 980.5672 \\
\hline 0.096907 & 1135.6051 & 0.152402 & 998.9428 & 0.098292 & 984.577 \\
\hline 0.148759 & 1161.0536 & 0.201804 & 1016.8568 & 0.147898 & 1004.6138 \\
\hline 0.195383 & 1175.3787 & 0.253663 & 1033.0431 & 0.197011 & 1016.7227 \\
\hline 0.245733 & 1187.6145 & 0.304384 & 1047.5642 & 0.24797 & 1025.9401 \\
\hline 0.298395 & 1197.769 & 0.356519 & 1062.1794 & 0.300768 & 1033.7214 \\
\hline 0.351764 & 1206.5123 & 0.408878 & 1075.502 & 0.345476 & 1039.5475 \\
\hline 0.395884 & 1213.0098 & 0.458997 & 1090.9355 & 0.402046 & 1046.3 \\
\hline 0.450173 & 1220.2767 & 0.510798 & 1104.5641 & 0.447467 & 1051.3531 \\
\hline 0.504374 & 1226.8685 & 0.564151 & 1118.7623 & 0.501464 & 1056.7881 \\
\hline 0.547022 & 1232.3408 & 0.615488 & 1134.114 & 0.548738 & 1061.2953 \\
\hline 0.599176 & 1238.7592 & 0.666195 & 1146.9523 & 0.598925 & 1065.1213 \\
\hline 0.649378 & 1244.9949 & 0.717402 & 1161.5262 & 0.645913 & 1069.0441 \\
\hline 0.696812 & 1249.7391 & 0.768108 & 1176.6283 & 0.69568 & 1073.1938 \\
\hline 0.745881 & 1255.6348 & 0.816074 & 1189.0067 & 0.747257 & 1077.2639 \\
\hline 0.797114 & 1262.1182 & 0.865947 & 1206.6393 & 0.797091 & 1080.2247 \\
\hline 0.849997 & 1268.4786 & 0.910237 & 1227.7894 & 0.846998 & 1083.7308 \\
\hline 0.90204 & 1276.9884 & 0.957819 & 1252.4851 & 0.900318 & 1088.1472 \\
\hline 0.948554 & 1290.4202 & 0.925034 & 1240.4166 & 0.947909 & 1091.0764 \\
\hline 0.927605 & 1288.8036 & 0.873588 & 1216.7278 & 0.926721 & 1090.851 \\
\hline 0.867956 & 1279.132 & 0.821221 & 1195.6902 & 0.864053 & 1087.0275 \\
\hline 0.828121 & 1276.6202 & 0.772306 & 1185.0444 & 0.825266 & 1084.9209 \\
\hline 0.764524 & 1271.1572 & 0.722998 & 1170.1017 & 0.771748 & 1082.301 \\
\hline 0.727612 & 1269.9059 & 0.673401 & 1154.4656 & 0.715295 & 1078.4882 \\
\hline 0.669016 & 1264.295 & 0.623231 & 1139.7428 & 0.667204 & 1074.6821 \\
\hline 0.616833 & 1259.6711 & 0.573112 & 1124.6141 & 0.617355 & 1070.4659 \\
\hline
\end{tabular}




\begin{tabular}{|l|l|l|l|l|l|}
\hline 0.569428 & 1254.0264 & 0.52611 & 1111.3406 & 0.567485 & 1066.9717 \\
\hline 0.517311 & 1248.3279 & 0.47415 & 1096.8984 & 0.515518 & 1062.126 \\
\hline 0.470783 & 1241.4028 & 0.423175 & 1080.8223 & 0.472627 & 1058.1346 \\
\hline 0.418342 & 1234.1581 & 0.371838 & 1066.9349 & 0.420785 & 1052.3376 \\
\hline 0.369627 & 1225.8427 & 0.320986 & 1053.3618 & 0.36979 & 1046.1184 \\
\hline 0.321199 & 1216.2683 & 0.270229 & 1039.1361 & 0.316345 & 1038.9817 \\
\hline 0.271711 & 1205.5701 & 0.222771 & 1024.8262 & 0.268019 & 1031.6616 \\
\hline 0.224151 & 1193.8911 & 0.174587 & 1008.3885 & 0.222038 & 1023.4851 \\
\hline 0.179103 & 1180.5044 & 0.127158 & 988.4124 & 0.177853 & 1013.8079 \\
\hline 0.129424 & 1160.6016 & 0.071753 & 949.2584 & & \\
\hline 0.07766 & 1123.3379 & 0.028993 & 756.0954 & & \\
\hline & & 0.019517 & 696.4122 & & \\
\hline
\end{tabular}

THF RSE samples

Table S38. $\mathrm{N}_{2}$ isotherm data for THF RSE FJI-1 samples used.

\begin{tabular}{|l|l|l|l|l|l|}
\hline THF RSE 1 & THF RSE 2 & & THF RSE 3 & \\
\hline $\mathrm{P}^{\mathrm{P}_{0}}$ & $\begin{array}{l}\mathrm{N}_{2} \text { uptake } \\
\left(\mathrm{cc} \mathrm{g}^{-1}\right)\end{array}$ & $\mathrm{P} / \mathrm{P}_{0}$ & $\begin{array}{l}\mathrm{N}_{2} \text { uptake } \\
\left(\mathrm{cc} \mathrm{g}^{-1}\right)\end{array}$ & $\mathrm{P} / \mathrm{P}_{0}$ & $\begin{array}{l}\mathrm{N}_{2} \text { uptake } \\
\left(\mathrm{cc} \mathrm{g}^{-1}\right)\end{array}$ \\
\hline 0.001535 & 182.3229 & 0.000772 & 106.4441 & 0.001605 & 200.8439 \\
\hline 0.002166 & 236.3549 & 0.001544 & 165.7249 & 0.002113 & 244.0178 \\
\hline 0.002188 & 241.2293 & 0.002191 & 220.6822 & 0.002158 & 247.8574 \\
\hline 0.002247 & 246.753 & 0.002213 & 225.6445 & 0.002224 & 252.3132 \\
\hline 0.002299 & 252.8673 & 0.002287 & 231.3021 & 0.002283 & 257.2134 \\
\hline 0.002344 & 259.4762 & 0.002323 & 237.6319 & 0.002334 & 262.4584 \\
\hline 0.003056 & 379.4238 & 0.002404 & 244.3639 & 0.003078 & 371.4006 \\
\hline 0.004279 & 559.6526 & 0.003037 & 336.2885 & 0.004013 & 545.485 \\
\hline 0.005051 & 601.0975 & 0.004228 & 517.3994 & 0.005228 & 642.2209 \\
\hline 0.010657 & 686.5416 & 0.005338 & 578.0984 & 0.010251 & 734.7576 \\
\hline 0.016071 & 725.8309 & 0.011963 & 666.5942 & 0.017062 & 787.2959 \\
\hline 0.020365 & 753.507 & 0.015639 & 693.9434 & 0.02 & 807.3572 \\
\hline 0.02503 & 783.4073 & 0.020139 & 722.9889 & 0.025163 & 842.6915 \\
\hline 0.031245 & 823.7968 & 0.025007 & 753.197 & 0.030074 & 875.3109 \\
\hline 0.035925 & 855.7778 & 0.03097 & 790.554 & 0.036481 & 920.0952 \\
\hline 0.040278 & 889.1393 & 0.036073 & 824.2997 & 0.041186 & 966.1349 \\
\hline 0.046582 & 940.2556 & 0.040624 & 857.5499 & 0.045958 & 1008.5301 \\
\hline 0.050001 & 962.8062 & 0.04547 & 896.3201 & 0.051282 & 1046.1095 \\
\hline 0.055652 & 986.8439 & 0.050632 & 930.5431 & 0.055723 & 1066.7407 \\
\hline 0.060614 & 1000.187 & 0.057132 & 956.8892 & 0.061526 & 1082.8157 \\
\hline 0.067511 & 1012.2955 & 0.060065 & 965.1164 & 0.06668 & 1092.6122 \\
\hline 0.071968 & 1018.0699 & 0.066433 & 976.4553 & 0.070215 & 1097.7917 \\
\hline 0.076032 & 1022.5468 & 0.070528 & 982.5298 & 0.076828 & 1105.752 \\
\hline 0.082128 & 1028.3251 & 0.077389 & 990.403 & 0.082388 & 1111.0746 \\
\hline 0.087631 & 1032.6671 & 0.083183 & 995.3699 & 0.086335 & 1114.561 \\
\hline
\end{tabular}




\begin{tabular}{|c|c|c|c|c|c|}
\hline 0.094128 & 1037.2271 & 0.088124 & 999.613 & 0.092491 & 1119.2744 \\
\hline 0.100528 & 1041.1971 & 0.09466 & 1004.121 & 0.097042 & 1122.4153 \\
\hline 0.146739 & 1061.8629 & 0.100785 & 1008.1136 & 0.145651 & 1144.6128 \\
\hline 0.198371 & 1076.5372 & 0.147049 & 1028.0883 & 0.20239 & 1159.9677 \\
\hline 0.247312 & 1086.6464 & 0.199049 & 1042.4504 & 0.248489 & 1167.8738 \\
\hline 0.301466 & 1096.0112 & 0.246048 & 1052.671 & 0.299859 & 1175.8037 \\
\hline 0.346475 & 1102.6329 & 0.302856 & 1062.1525 & 0.347467 & 1181.1495 \\
\hline 0.400963 & 1110.0598 & 0.347708 & 1069.3831 & 0.396636 & 1186.9139 \\
\hline 0.446922 & 1115.306 & 0.396222 & 1076.2952 & 0.447381 & 1191.631 \\
\hline 0.502359 & 1122.524 & 0.445508 & 1083.0597 & 0.495166 & 1195.9402 \\
\hline 0.547428 & 1127.6791 & 0.496566 & 1089.4083 & 0.552516 & 1200.5768 \\
\hline 0.595878 & 1132.8875 & 0.551573 & 1097.196 & 0.598659 & 1204.4969 \\
\hline 0.65024 & 1138.9189 & 0.598859 & 1102.0975 & 0.647069 & 1209.0868 \\
\hline 0.697652 & 1143.2468 & 0.648506 & 1107.8867 & 0.699147 & 1210.6692 \\
\hline 0.749596 & 1148.0798 & 0.69924 & 1113.7131 & 0.747992 & 1215.4491 \\
\hline 0.80085 & 1152.6409 & 0.748004 & 1119.5137 & 0.79842 & 1220.4927 \\
\hline 0.848426 & 1156.7876 & 0.799423 & 1124.1636 & 0.847994 & 24.1757 \\
\hline 0.897885 & 1161.3101 & 0.848789 & 1128.9988 & 0.901125 & 28.2305 \\
\hline 0.948346 & 1167.8605 & 0.89 & 1137 & 353 & 1752 \\
\hline 0.924985 & 3181 & 0.9 & 1143 & 373 & 3.3121 \\
\hline 0.87 & 075 & $0.9 c$ & 114 & 0.8 & 1229.5812 \\
\hline $0.82 \varepsilon$ & 6378 & 0.86 & 1139 & $0.8 ?$ & 0.3491 \\
\hline 0.770599 & 1152.8451 & 0.82 & 1135 & 0.77 & 3.4913 \\
\hline 0.729142 & 1148.7817 & 0.76 & 1131 & $0.7 t$ & .8113 \\
\hline 0.66686 & 1143. & 0.72 & 1130. & 0.66 & 3.0002 \\
\hline 0.628406 & 1140.8831 & & & 0.61 & 1213.3268 \\
\hline 0.569372 & 1134.4578 & & & 0.568599 & 1208.8099 \\
\hline 0.518511 & 1129.225 & & & 0.528326 & 1206.2513 \\
\hline 0.470862 & 1123.4624 & & & 0.472558 & 1199.6836 \\
\hline 0.422507 & 1116.0465 & & & 0.420864 & 1194.5415 \\
\hline 0.364623 & 1107.7861 & & & 0.379802 & 1190.4824 \\
\hline 0.320266 & 1100.7645 & & & 0.319182 & 1183.2144 \\
\hline 0.271941 & 1092.415 & & & 0.267355 & 1174.2433 \\
\hline 0.221903 & 1082.1974 & & & 0.221073 & 1165.8156 \\
\hline 0.175996 & 1070.6313 & & & 0.176322 & 1155.2343 \\
\hline 0.125803 & 1052.7997 & & & 0.12744 & 1138.5342 \\
\hline 0.073792 & 1017.4614 & & & 0.076828 & 1105.4358 \\
\hline 0.028723 & 801.5673 & & & 0.028911 & 870.475 \\
\hline 0.01883 & 735.8391 & & & 0.019816 & 805.8362 \\
\hline
\end{tabular}


DMF RSE samples

Table S39. $\mathrm{N}_{2}$ isotherm data for DMF RSE FJI-1 samples used.

\begin{tabular}{|c|c|c|c|c|c|}
\hline DMF RSE 1 & & DMF RSE 2 & & DMF RSE 3 & \\
\hline $\mathrm{P} / \mathrm{P}_{0}$ & $\begin{array}{l}N_{2} \text { uptake } \\
\left(\mathrm{cc} \mathrm{g}^{-1}\right)\end{array}$ & $\mathrm{P} / \mathrm{P}_{0}$ & $\begin{array}{l}\mathrm{N}_{2} \text { uptake } \\
\left(\mathrm{cc} \mathrm{g}^{-1}\right)\end{array}$ & $\mathrm{P} / \mathrm{P}_{0}$ & $\begin{array}{l}\mathrm{N}_{2} \text { uptake } \\
\left(\mathrm{cc} \mathrm{g}^{-1}\right)\end{array}$ \\
\hline 0.002083 & 264.7953 & 0.001551 & 192.291 & 0.00162 & 230.5477 \\
\hline 0.002054 & 267.3467 & 0.002156 & 254.662 & 0.002117 & 293.2907 \\
\hline 0.002061 & 270.1726 & 0.002178 & 260.2812 & 0.002095 & 295.9899 \\
\hline 0.002075 & 273.3235 & 0.002223 & 266.6719 & 0.002117 & 298.9921 \\
\hline 0.002097 & 276.8842 & 0.002274 & 273.8261 & 0.002138 & 302.3972 \\
\hline 0.002104 & 280.6009 & 0.002326 & 281.3679 & 0.002153 & 306.0447 \\
\hline 0.003038 & 468.6271 & 0.003064 & 418.8617 & 0.003038 & 484.5482 \\
\hline 0.004144 & 586.1218 & 0.004135 & 554.8927 & 0.004133 & 613.2234 \\
\hline 0.005513 & 634.6876 & 0.005184 & 602.7428 & 0.00522 & 659.7482 \\
\hline 0.010577 & 698.8628 & 0.011947 & 687.7316 & 0.010894 & 739.359 \\
\hline 0.016754 & 742.1859 & 0.016097 & 716.3025 & 0.016294 & 780.1926 \\
\hline 0.020177 & 764.1716 & 0.02038 & 744.4138 & 0.021319 & 815.0885 \\
\hline 0.026097 & 801.9724 & 0.026368 & 782.3812 & 0.025639 & 845.2242 \\
\hline 0.030704 & 831.8817 & 0.031101 & 813.0072 & 0.030226 & 877.1441 \\
\hline 0.03524 & 862.6147 & 0.03534 & 842.0678 & 0.036115 & 921.4629 \\
\hline 0.04141 & 910.6229 & 0.040531 & 882.4495 & 0.041148 & 965.1177 \\
\hline 0.045175 & 941.7403 & 0.045589 & 924.2341 & 0.045943 & 1006.8843 \\
\hline 0.051944 & 983.1604 & 0.051267 & 959.6166 & 0.050558 & 1037.5962 \\
\hline 0.055603 & 997.1359 & 0.055203 & 975.0876 & 0.05503 & 1056.475 \\
\hline 0.060303 & 1008.7639 & 0.060748 & 989.2214 & 0.060429 & 1070.392 \\
\hline 0.066394 & 1019.146 & 0.066589 & 999.7262 & 0.065851 & 1080.3301 \\
\hline 0.070901 & 1024.9648 & 0.071654 & 1006.4565 & 0.070884 & 1087.3451 \\
\hline 0.076293 & 1030.6963 & 0.07779 & 1012.604 & 0.075139 & 1092.296 \\
\hline 0.080266 & 1034.3364 & 0.083173 & 1018.3118 & 0.080143 & 1097.2168 \\
\hline 0.086435 & 1039.3074 & 0.088903 & 1022.3619 & 0.085665 & 1101.9874 \\
\hline 0.091884 & 1043.1865 & 0.095579 & 1026.5819 & 0.092477 & 1107.1632 \\
\hline 0.097654 & 1046.834 & 0.100607 & 1029.3478 & 0.098345 & 1111.1588 \\
\hline 0.148956 & 1069.2197 & 0.145317 & 1049.96 & 0.14598 & 1132.6626 \\
\hline 0.196571 & 1081.7429 & 0.196392 & 1065.3804 & 0.196437 & 1146.8229 \\
\hline 0.24778 & 1092.8751 & 0.2478 & 1076.6194 & 0.249134 & 1157.8087 \\
\hline 0.29742 & 1101.4547 & 0.298845 & 1086.3588 & 0.303832 & 1166.9723 \\
\hline 0.345655 & 1109.4773 & 0.347395 & 1093.0027 & 0.358437 & 1175.3383 \\
\hline 0.397043 & 1117.7875 & 0.395421 & 1099.9775 & 0.410198 & 1182.6469 \\
\hline 0.450299 & 1124.8096 & 0.446983 & 1105.8363 & 0.464313 & 1188.751 \\
\hline 0.501579 & 1131.6493 & 0.497859 & 1112.1667 & 0.516902 & 1194.7672 \\
\hline 0.552896 & 1138.6382 & 0.548587 & 1117.5389 & 0.569145 & 1200.3966 \\
\hline 0.601994 & 1145.6323 & 0.596753 & 1122.7767 & 0.619249 & 1205.8208 \\
\hline 0.651334 & 1152.4739 & 0.649142 & 1129.4834 & 0.673552 & 1211.4233 \\
\hline
\end{tabular}




\begin{tabular}{|l|l|l|l|l|l|}
\hline 0.701117 & 1159.6505 & 0.698084 & 1134.4274 & 0.724614 & 1216.6818 \\
\hline 0.749794 & 1166.509 & 0.747438 & 1140.486 & 0.776727 & 1222.4092 \\
\hline 0.798764 & 1173.759 & 0.79536 & 1147.4038 & 0.825918 & 1227.1097 \\
\hline 0.847755 & 1181.4056 & 0.847358 & 1152.7528 & 0.87603 & 1233.5425 \\
\hline 0.899913 & 1188.5674 & 0.899445 & 1160.5803 & 0.925832 & 1240.5527 \\
\hline 0.94864 & 1196.0598 & 0.947729 & 1166.5249 & 0.966361 & 1253.1782 \\
\hline 0.917979 & 1192.351 & 0.927911 & 1166.9775 & 0.922081 & 1247.1144 \\
\hline 0.872418 & 1187.3215 & 0.866321 & 1160.3065 & 0.870349 & 1239.7775 \\
\hline 0.824362 & 1180.8674 & 0.828928 & 1156.6244 & 0.819618 & 1233.645 \\
\hline 0.774201 & 1174.3966 & 0.765691 & 1151.6769 & 0.770881 & 1228.5225 \\
\hline 0.724154 & 1167.3201 & 0.727701 & 1146.7703 & 0.718055 & 1222.1968 \\
\hline 0.675662 & 1160.9226 & 0.664198 & 1144.396 & 0.669779 & 1216.6943 \\
\hline 0.62655 & 1154.0848 & 0.628342 & 1140.6165 & 0.620063 & 1210.8489 \\
\hline 0.579249 & 1147.9271 & 0.568612 & 1133.0107 & 0.571139 & 1205.7172 \\
\hline 0.528675 & 1140.6802 & 0.51623 & 1126.8617 & 0.523533 & 1200.6841 \\
\hline 0.476253 & 1133.6453 & 0.478166 & 1121.2869 & 0.474832 & 1185.3737 \\
\hline 0.425193 & 1126.641 & 0.420681 & 1112.8522 & 0.4222 & 1179.532 \\
\hline 0.376859 & 1119.3867 & 0.36965 & 1105.1984 & 0.373737 & 1173.7726 \\
\hline 0.325842 & 1110.6116 & 0.322016 & 1097.6135 & 0.321076 & 1165.7828 \\
\hline 0.278106 & 1101.7905 & 0.267508 & 1084.4019 & 0.274068 & 1158.5696 \\
\hline 0.228694 & 1092.1434 & 0.220449 & 1073.9969 & 0.22366 & 1149.47 \\
\hline 0.173362 & 1078.1007 & 0.175355 & 1059.7216 & 0.177062 & 1139.2722 \\
\hline 0.126055 & 1061.8915 & 0.124583 & 1039.8478 & 0.122781 & 1121.8374 \\
\hline 0.071208 & 1024.3174 & 0.073028 & 1003.7943 & 0.072173 & 1087.8386 \\
\hline 0.029791 & 822.3125 & 0.029913 & 801.2363 & 0.029182 & 870.3054 \\
\hline 0.019592 & 754.9392 & 0.018615 & 726.1517 & 0.019296 & 800.5732 \\
\hline
\end{tabular}

DMSO RSE samples

Table S40. $\mathrm{N}_{2}$ isotherm data for DMSO RSE FJI-1 samples used.

\begin{tabular}{|l|l|l|l|l|l|}
\hline $\begin{array}{l}\text { DMSO } \\
\text { RSE 1 }\end{array}$ & $\begin{array}{l}\text { DMSO } \\
\text { RSE 2 }\end{array}$ & $\begin{array}{l}\text { DMSO } \\
\text { RSE 3 }\end{array}$ & \\
\hline $\mathrm{P}^{\mathrm{P}_{0}}$ & $\begin{array}{l}\mathrm{N}_{2} \text { uptake } \\
\left(\mathrm{cc} \mathrm{g}^{-1}\right)\end{array}$ & $\mathrm{P} / \mathrm{P}_{0}$ & $\begin{array}{l}\mathrm{N}_{2} \text { uptake } \\
\left(\mathrm{cc} \mathrm{g}^{-1}\right)\end{array}$ & $\mathrm{P} / \mathrm{P}_{0}$ & $\begin{array}{l}\mathrm{N}_{2} \text { uptake } \\
\left(\mathrm{cc} \mathrm{g}^{-1}\right)\end{array}$ \\
\hline 0.000869 & 131.121 & 0.00103 & 124.6889 & 0.001528 & 151.4394 \\
\hline 0.001524 & 179.2103 & 0.00164 & 164.2211 & 0.002167 & 197.8713 \\
\hline 0.002187 & 231.9364 & 0.00214 & 199.5647 & 0.002248 & 204.9084 \\
\hline 0.002224 & 236.6431 & 0.002206 & 203.2615 & 0.002359 & 212.861 \\
\hline 0.002275 & 242.0457 & 0.00225 & 207.3293 & 0.002463 & 221.7717 \\
\hline 0.002297 & 247.9041 & 0.002309 & 211.8364 & 0.002575 & 231.2417 \\
\hline 0.002349 & 254.2601 & 0.002375 & 216.8711 & 0.003012 & 284.4106 \\
\hline 0.003144 & 372.7437 & 0.003082 & 307.0381 & 0.004059 & 425.1827 \\
\hline 0.004109 & 517.897 & 0.004111 & 442.5896 & 0.005105 & 486.2153 \\
\hline 0.005059 & 582.1251 & 0.005494 & 508.3938 & 0.013036 & 582.29 \\
\hline
\end{tabular}




\begin{tabular}{|c|c|c|c|c|c|}
\hline 0.012422 & 693.2261 & 0.010973 & 572.9513 & 0.01619 & 601.6815 \\
\hline 0.015706 & 718.0737 & 0.015812 & 602.8269 & 0.020456 & 624.7291 \\
\hline 0.020948 & 752.166 & 0.021122 & 631.7404 & 0.025316 & 649.9211 \\
\hline 0.026073 & 784.4592 & 0.025939 & 657.0793 & 0.030094 & 675.522 \\
\hline 0.031242 & 817.9498 & 0.030933 & 683.9539 & 0.036149 & 708.2744 \\
\hline 0.036124 & 850.6786 & 0.0355 & 709.1949 & 0.041491 & 741.35 \\
\hline 0.040984 & 887.2243 & 0.041192 & 744.9642 & 0.045698 & 772.9135 \\
\hline 0.045497 & 923.2094 & 0.045223 & 772.1351 & 0.051292 & 804.9429 \\
\hline 0.050225 & 954.6838 & 0.052003 & 807.5397 & 0.056375 & 824.5592 \\
\hline 0.056535 & 981.1003 & 0.055997 & 821.0007 & 0.060723 & 835.2303 \\
\hline 0.061004 & 993.3001 & 0.061116 & 831.6889 & 0.0653 & 843.1642 \\
\hline 0.065938 & 1002.8864 & 0.06658 & 839.8558 & 0.071058 & 850.3308 \\
\hline 0.072609 & 1012.2228 & 0.070492 & 844.4086 & 0.077832 & 856.1341 \\
\hline 0.077513 & 1018.0887 & 0.075486 & 849.2436 & 0.085905 & 860.1937 \\
\hline 0.082888 & 1023.4689 & 0.081414 & 854.062 & 0.090921 & 862.8665 \\
\hline 0.087865 & 1027.7866 & 0.086488 & 857.6321 & 0.097576 & 866.7142 \\
\hline 0.092482 & 1031.4115 & 0.093071 & 861.684 & 0.103771 & 870.115 \\
\hline 0.098167 & 1035.5341 & 0.099646 & 865.2483 & 0.150011 & 888.0184 \\
\hline 0.149687 & 1061.6733 & 0.148854 & 883.3126 & 0.195746 & 899.747 \\
\hline 0.198712 & 1076.9551 & 0.19554 & 894.7459 & 0.246288 & 909.3829 \\
\hline 0.247375 & 1089.0079 & 0.248485 & 903.7646 & 0.296891 & 916.0774 \\
\hline 0.300655 & 1100.4734 & 0.299407 & 911.564 & 0.345801 & 923.6879 \\
\hline 0.34845 & 1109.4686 & 0.347138 & 917.2921 & 0.399237 & 930.5933 \\
\hline 0.39637 & 1116.6212 & 0.401451 & 923.5388 & 0.44935 & 936.1876 \\
\hline 0.449562 & 1125.9209 & 0.447313 & 928.4464 & 0.499767 & 940.0232 \\
\hline 0.500221 & 1134.984 & 0.502243 & 933.7645 & 0.547067 & 946.0948 \\
\hline 0.54697 & 1142.9274 & 0.546885 & 938.5574 & 0.597625 & 952.0197 \\
\hline 0.595825 & 1145.9629 & 0.598182 & 942.0143 & 0.647655 & 954.7691 \\
\hline 0.651823 & 1153.5006 & 0.645641 & 946.0782 & 0.697145 & 957.4207 \\
\hline 0.696532 & 1160.0045 & 0.697673 & 949.5361 & 0.749082 & 962.2063 \\
\hline 0.747964 & 1166.4342 & 0.75128 & 954.7514 & 0.801406 & 962.4742 \\
\hline 0.797563 & 1173.5105 & 0.806144 & 957.3516 & 0.850183 & 966.5148 \\
\hline 0.854584 & 1182.3998 & 0.848278 & 961.3093 & 0.904279 & 972.1477 \\
\hline 0.905309 & 1188.1936 & 0.895317 & 965.0455 & 0.952114 & 976.4901 \\
\hline 0.948708 & 1196.6942 & 0.954888 & 970.8543 & 0.927332 & 973.7441 \\
\hline 0.925963 & 1196.1644 & 0.928177 & 969.0175 & 0.867166 & 970.6229 \\
\hline 0.866358 & 1193.1174 & 0.868863 & 966.3304 & 0.825037 & 973.0392 \\
\hline 0.828592 & 1192.4664 & 0.813624 & 961.6224 & 0.763862 & 972.0514 \\
\hline 0.770886 & 1186.7369 & 0.777918 & 960.0528 & 0.728559 & 974.9474 \\
\hline 0.717989 & 1183.9153 & 0.715802 & 955.9575 & 0.664661 & 970.3545 \\
\hline 0.67967 & 1180.4277 & 0.677897 & 953.1222 & 0.628661 & 969.8063 \\
\hline 0.619432 & 1173.1696 & 0.617142 & 949.0406 & 0.567033 & 966.1333 \\
\hline 0.57149 & 1168.3765 & 0.566926 & 945.1307 & 0.51632 & 959.114 \\
\hline
\end{tabular}




\begin{tabular}{|l|l|l|l|l|l|}
\hline 0.518659 & 1160.769 & 0.514739 & 940.504 & 0.479592 & 955.3912 \\
\hline 0.470083 & 1152.329 & 0.474804 & 935.369 & 0.417297 & 947.3987 \\
\hline 0.42206 & 1143.7167 & 0.422175 & 930.4109 & 0.363415 & 940.4114 \\
\hline 0.372064 & 1134.1661 & 0.36773 & 923.7932 & 0.317324 & 931.6031 \\
\hline 0.320823 & 1123.0796 & 0.321206 & 918.3904 & 0.268585 & 922.9334 \\
\hline 0.269988 & 1110.2961 & 0.271019 & 910.5769 & 0.218079 & 912.3029 \\
\hline 0.223172 & 1097.2108 & 0.221693 & 902.0933 & 0.175282 & 900.77 \\
\hline 0.175127 & 1081.7739 & 0.174977 & 892.2562 & 0.123315 & 881.0806 \\
\hline 0.125771 & 1060.3378 & & & 0.077135 & 851.7094 \\
\hline & & & & 0.026421 & 653.189 \\
\hline & & & & 0.018289 & 608.1123 \\
\hline
\end{tabular}

\section{UiO-66}

\section{Pristine samples}

Table S41. $\mathrm{N}_{2}$ isotherm data for pristine UiO-66 samples used. Samples are labelled with their BET surface areas, given in parentheses.

\begin{tabular}{|l|l|l|l|}
\hline Pristine (1648.81) & & Pristine (1544.766) & \\
\hline $\mathrm{P}^{\mathrm{P}} \mathrm{P}_{0}$ & $\begin{array}{l}\mathrm{N}_{2} \text { uptake } \\
\left(\mathrm{cc} \mathrm{g}^{-1}\right)\end{array}$ & $\mathrm{P}^{\mathrm{P}} \mathrm{P}_{0}$ & $\begin{array}{l}\mathrm{N}_{2} \text { uptake } \\
\left(\mathrm{cc} \mathrm{g}^{-1}\right)\end{array}$ \\
\hline 0.002938 & 216.7104 & 0.001761 & 200.7243 \\
\hline 0.003555 & 222.7127 & 0.002163 & 207.7869 \\
\hline 0.004304 & 228.9196 & 0.002293 & 209.8184 \\
\hline 0.005163 & 235.4742 & 0.002473 & 211.9499 \\
\hline 0.006059 & 241.607 & 0.002638 & 214.2853 \\
\hline 0.007139 & 248.082 & 0.002868 & 216.89 \\
\hline 0.008219 & 254.3458 & 0.003112 & 219.6385 \\
\hline 0.009365 & 260.0793 & 0.00414 & 229.3341 \\
\hline 0.010532 & 265.3442 & 0.005376 & 238.8598 \\
\hline 0.013412 & 276.4538 & 0.011457 & 269.115 \\
\hline 0.01715 & 288.3472 & 0.015913 & 285.3063 \\
\hline 0.021271 & 299.9169 & 0.020959 & 301.1307 \\
\hline 0.026023 & 312.0817 & 0.025753 & 315.1251 \\
\hline 0.030569 & 323.2985 & 0.030914 & 328.128 \\
\hline 0.036173 & 335.7427 & 0.03537 & 337.0929 \\
\hline 0.041461 & 347.0021 & 0.041738 & 346.8474 \\
\hline 0.04636 & 356.0132 & 0.046101 & 351.9967 \\
\hline 0.052802 & 366.1554 & 0.050989 & 356.8105 \\
\hline 0.058619 & 373.4695 & 0.055553 & 360.3582 \\
\hline 0.064781 & 379.6046 & 0.061116 & 364.245 \\
\hline 0.0704 & 384.0757 & 0.065946 & 366.9864 \\
\hline 0.077627 & 388.7356 & 0.070927 & 369.5344 \\
\hline 0.083077 & 391.8799 & 0.076979 & 372.1947 \\
\hline & & &
\end{tabular}




\begin{tabular}{|l|l|l|l|}
\hline 0.087006 & 393.9419 & 0.082815 & 374.4933 \\
\hline 0.093279 & 396.6494 & 0.089147 & 376.7411 \\
\hline 0.099683 & 398.999 & 0.096809 & 379.1248 \\
\hline 0.104142 & 400.5673 & 0.102523 & 380.774 \\
\hline 0.1512 & 412.2884 & 0.146806 & 390.2991 \\
\hline 0.199668 & 420.6237 & 0.195883 & 398.1253 \\
\hline 0.251243 & 427.7198 & 0.248402 & 405.5077 \\
\hline 0.301886 & 434.214 & 0.299082 & 412.0729 \\
\hline 0.349135 & 440.1493 & 0.348691 & 418.3297 \\
\hline 0.397316 & 446.0227 & 0.401059 & 424.5107 \\
\hline 0.448231 & 452.624 & 0.450847 & 431.19 \\
\hline 0.499879 & 458.0694 & 0.501297 & 437.4467 \\
\hline 0.54682 & 464.1263 & 0.552573 & 443.8763 \\
\hline 0.599886 & 468.6935 & 0.60193 & 450.6916 \\
\hline 0.649536 & 475.02 & 0.650755 & 457.1011 \\
\hline 0.698636 & 482.2197 & 0.702103 & 463.9814 \\
\hline 0.750777 & 486.312 & 0.751647 & 471.0137 \\
\hline 0.80092 & 492.5435 & 0.801615 & 478.2235 \\
\hline 0.849403 & 499.8122 & 0.85039 & 485.5353 \\
\hline 0.898033 & 509.2918 & 0.900667 & 496.6621 \\
\hline 0.947412 & 517.223 & 0.948119 & 508.9101 \\
\hline 0.924503 & 515.8483 & 0.925277 & 502.1623 \\
\hline 0.879678 & 509.3445 & 0.878989 & 491.4983 \\
\hline 0.821948 & 502.0055 & 0.829424 & 479.9225 \\
\hline 0.779591 & 499.0551 & 0.77914 & 473.4005 \\
\hline 0.717013 & 493.5852 & 0.728144 & 465.6208 \\
\hline 0.677674 & 489.6619 & 0.677673 & 458.5453 \\
\hline 0.6178 & 484.5073 & 0.62575 & 451.7275 \\
\hline 0.566717 & 479.8353 & 0.577334 & 445.5444 \\
\hline 0.514253 & 473.6493 & 0.527977 & 439.4277 \\
\hline 0.477426 & 469.1308 & 0.473977 & 433.3356 \\
\hline 0.423163 & 462.4883 & 0.424958 & 427.2081 \\
\hline 0.365455 & 454.675 & 0.374666 & 421.2119 \\
\hline 0.313696 & 446.5221 & 0.327056 & 415.6129 \\
\hline 0.266616 & 439.4718 & 0.274953 & 409.1849 \\
\hline 0.217024 & 430.7812 & 0.225115 & 402.2708 \\
\hline 0.168725 & 420.9124 & 0.177159 & 395.3254 \\
\hline 0.123209 & 409.4842 & 0.121822 & 385.4583 \\
\hline 0.070789 & 385.2401 & 0.074995 & 371.9686 \\
\hline 0.025508 & 308.9412 & 0.029189 & 326.2901 \\
\hline 0.019699 & 291.8843 & 0.019148 & 296.7492 \\
\hline & & & \\
\hline & & & \\
\hline
\end{tabular}


EtOH resolvated samples

Table S42. $\mathrm{N}_{2}$ isotherm data for $\mathrm{EtOH}$ resolvated UiO-66 samples used.

\begin{tabular}{|c|c|c|c|c|c|}
\hline $\begin{array}{l}\text { EtOH } \\
\text { resolvation } 1\end{array}$ & & $\begin{array}{l}\text { EtOH } \\
\text { resolvation } 2\end{array}$ & & $\begin{array}{l}\mathrm{EtOH} \\
\text { resolvation } 3\end{array}$ & \\
\hline $\mathrm{P} / \mathrm{P}_{0}$ & $\begin{array}{l}\mathrm{N}_{2} \text { uptake } \\
\left(\mathrm{cc} \mathrm{g}^{-1}\right)\end{array}$ & $\mathrm{P} / \mathrm{P}_{0}$ & $\begin{array}{l}\mathrm{N}_{2} \text { uptake } \\
\left(\mathrm{cc} \mathrm{g}^{-1}\right)\end{array}$ & $\mathrm{P} / \mathrm{P}_{0}$ & $\begin{array}{l}\mathrm{N}_{2} \text { uptake } \\
\left(\mathrm{cc} \mathrm{g} \mathrm{g}^{-1}\right)\end{array}$ \\
\hline 0.001854 & 206.3747 & 0.000827 & 175.846 & 0.001153 & 203.3106 \\
\hline 0.002251 & 212.3593 & 0.001547 & 198.4167 & 0.001574 & 215.6336 \\
\hline 0.002764 & 218.6912 & 0.002065 & 206.9809 & 0.002299 & 228.0148 \\
\hline 0.003391 & 224.9926 & 0.002353 & 210.9609 & 0.002516 & 231.5761 \\
\hline 0.004099 & 231.2256 & 0.002705 & 214.9926 & 0.002821 & 235.2695 \\
\hline 0.004892 & 237.4981 & 0.003123 & 219.3833 & 0.003183 & 239.1382 \\
\hline 0.005867 & 243.9046 & 0.003619 & 223.8601 & 0.003604 & 243.5079 \\
\hline 0.00692 & 250.0238 & 0.004187 & 228.4298 & 0.004119 & 248.0045 \\
\hline 0.008082 & 256.1658 & 0.004835 & 233.0923 & 0.004685 & 252.6755 \\
\hline 0.010687 & 268.031 & 0.005626 & 238.1045 & 0.005337 & 257.4665 \\
\hline 0.016034 & 288.5891 & 0.01121 & 264.0041 & 0.01095 & 286.1538 \\
\hline 0.021294 & 306.3652 & 0.016469 & 282.3591 & 0.015736 & 303.8608 \\
\hline 0.026901 & 322.1881 & 0.020124 & 293.8657 & 0.021392 & 322.147 \\
\hline 0.031216 & 331.8495 & 0.025714 & 310.0291 & 0.025018 & 332.6796 \\
\hline 0.036245 & 340.6806 & 0.03029 & 320.7651 & 0.030152 & 344.9318 \\
\hline 0.042494 & 348.7491 & 0.035801 & 330.9811 & 0.035598 & 354.9859 \\
\hline 0.048585 & 354.605 & 0.040593 & 337.7509 & 0.040116 & 361.3971 \\
\hline 0.055663 & 359.9106 & 0.045572 & 343.2325 & 0.045148 & 367.0558 \\
\hline 0.06158 & 363.6904 & 0.050579 & 347.6488 & 0.050355 & 371.7708 \\
\hline 0.068638 & 367.4807 & 0.056918 & 352.1296 & 0.05707 & 376.6264 \\
\hline 0.073833 & 369.9511 & 0.064149 & 356.3452 & 0.062581 & 380.0136 \\
\hline 0.078971 & 372.1403 & 0.070286 & 359.2913 & 0.068477 & 383.058 \\
\hline 0.085097 & 374.5304 & 0.075862 & 361.6052 & 0.074474 & 385.7689 \\
\hline 0.08934 & 375.8574 & 0.08128 & 363.689 & 0.081384 & 388.4741 \\
\hline 0.09574 & 377.6822 & 0.086604 & 365.4508 & 0.088049 & 390.8468 \\
\hline 0.101975 & 379.541 & 0.093799 & 367.7109 & 0.094698 & 393.0353 \\
\hline 0.10808 & 381.1396 & 0.099475 & 369.311 & 0.099941 & 394.6911 \\
\hline 0.159659 & 394.0309 & 0.106411 & 371.128 & 0.105525 & 396.3785 \\
\hline 0.213302 & 405.1084 & 0.156537 & 382.8336 & 0.155285 & 407.8639 \\
\hline 0.264809 & 415.2713 & 0.20934 & 393.2907 & 0.20944 & 417.8372 \\
\hline 0.318055 & 425.5337 & 0.261553 & 402.807 & 0.261862 & 426.5241 \\
\hline 0.369742 & 435.7633 & 0.313319 & 411.3442 & 0.312739 & 434.4246 \\
\hline 0.422353 & 445.5701 & 0.364388 & 419.5036 & 0.365762 & 442.7817 \\
\hline 0.474776 & 455.2865 & 0.415198 & 427.5283 & 0.418989 & 450.6761 \\
\hline 0.526255 & 465.2723 & 0.467806 & 435.7062 & 0.471352 & 458.9047 \\
\hline 0.576059 & 474.5344 & 0.519113 & 443.491 & 0.520221 & 466.1785 \\
\hline 0.626252 & 484.4568 & 0.568706 & 450.7203 & 0.570771 & 474.5713 \\
\hline
\end{tabular}




\begin{tabular}{|l|l|l|l|l|l|}
\hline 0.67514 & 494.3028 & 0.619286 & 458.3041 & 0.62072 & 482.81 \\
\hline 0.725211 & 504.6229 & 0.670498 & 465.5954 & 0.670771 & 491.0011 \\
\hline 0.774697 & 515.4197 & 0.721143 & 472.1541 & 0.719588 & 498.7291 \\
\hline 0.824718 & 527.0497 & 0.774291 & 480.674 & 0.768979 & 507.0543 \\
\hline 0.873945 & 539.7574 & 0.82233 & 487.5899 & 0.819442 & 515.8666 \\
\hline 0.921887 & 553.1544 & 0.872162 & 495.0663 & 0.868709 & 525.3276 \\
\hline 0.969866 & 574.4597 & 0.921828 & 503.9381 & 0.918136 & 536.1963 \\
\hline 0.927155 & 553.6141 & 0.966011 & 518.3292 & 0.963792 & 550.014 \\
\hline 0.871679 & 536.5826 & 0.92808 & 501.5455 & 0.926446 & 537.6813 \\
\hline 0.820425 & 522.3981 & 0.875363 & 488.8062 & 0.875345 & 525.4321 \\
\hline 0.770483 & 510.4491 & 0.823086 & 479.2901 & 0.822343 & 515.1664 \\
\hline 0.717779 & 498.3336 & 0.771247 & 470.6799 & 0.772822 & 506.086 \\
\hline 0.668306 & 489.3371 & 0.719366 & 461.3862 & 0.721415 & 497.1557 \\
\hline 0.619332 & 480.7845 & 0.669678 & 454.019 & 0.670524 & 489.0974 \\
\hline 0.571022 & 472.0006 & 0.621149 & 446.0977 & 0.620546 & 481.1416 \\
\hline 0.523123 & 463.4614 & 0.570246 & 440.2263 & 0.57323 & 473.3164 \\
\hline 0.472561 & 453.7315 & 0.521998 & 435.2037 & 0.523941 & 465.0881 \\
\hline 0.420838 & 443.8894 & 0.472548 & 428.5171 & 0.470003 & 456.9856 \\
\hline 0.369129 & 436.4757 & 0.42017 & 420.4151 & 0.420439 & 449.2489 \\
\hline 0.319137 & 428.9619 & 0.371756 & 412.4773 & 0.368061 & 441.6353 \\
\hline 0.267031 & 419.8664 & 0.31868 & 404.16 & 0.319562 & 434.0573 \\
\hline 0.217963 & 408.6025 & 0.267172 & 396.6887 & & \\
\hline 0.169025 & 396.4654 & 0.21765 & 388.8363 & & \\
\hline 0.121133 & 384.1444 & 0.170244 & 380.8334 & & \\
\hline 0.07327 & 367.9239 & 0.123506 & 371.7868 & & \\
\hline 0.026417 & 320.139 & 0.075776 & 358.5822 & & \\
\hline 0.018732 & 296.7393 & 0.02752 & 314.8941 & & \\
\hline & & & & & \\
\hline
\end{tabular}

DMF resolvated samples

Table S43. $\mathrm{N}_{2}$ isotherm data for DMF resolvated UiO-66 samples used.

\begin{tabular}{|l|l|l|l|l|l|}
\hline $\begin{array}{l}\text { DMF } \\
\text { resolvation 1 }\end{array}$ & $\begin{array}{l}\text { DMF } \\
\text { resolvation 2 }\end{array}$ & $\begin{array}{l}\text { DMF } \\
\text { resolvation 3 }\end{array}$ & \\
\hline $\mathrm{P}_{\mathrm{P}}$ & $\begin{array}{l}\mathrm{N}_{2} \text { uptake } \\
\left(\mathrm{cc} \mathrm{g}^{-1}\right)\end{array}$ & $\mathrm{P} / \mathrm{P}_{0}$ & $\begin{array}{l}\mathrm{N}_{2} \text { uptake } \\
\left(\mathrm{cc} \mathrm{g}^{-1}\right)\end{array}$ & $\mathrm{P} / \mathrm{P}_{0}$ & $\begin{array}{l}\mathrm{N}_{2} \text { uptake } \\
\left(\mathrm{cc} \mathrm{g}^{-1}\right)\end{array}$ \\
\hline 0.001652 & 209.5393 & 0.001072 & 199.3837 & 0.001073 & 200.1529 \\
\hline 0.002143 & 217.9328 & 0.001583 & 214.1244 & 0.001617 & 215.25 \\
\hline 0.002504 & 222.8206 & 0.002158 & 223.7468 & 0.002175 & 224.9534 \\
\hline 0.002951 & 228.1085 & 0.002461 & 227.9166 & 0.002451 & 229.3485 \\
\hline 0.003442 & 233.5323 & 0.002835 & 232.3746 & 0.002828 & 233.8445 \\
\hline 0.004041 & 239.349 & 0.003295 & 237.2173 & 0.003256 & 238.5464 \\
\hline 0.004777 & 245.2411 & 0.003828 & 242.1624 & 0.003793 & 243.7674 \\
\hline 0.005585 & 251.0581 & 0.004439 & 247.1862 & 0.004416 & 249.0871 \\
\hline 0.006523 & 256.8963 & 0.00513 & 252.3668 & 0.005134 & 254.5198 \\
\hline
\end{tabular}




\begin{tabular}{|c|c|c|c|c|c|}
\hline 0.010153 & 274.7454 & 0.005957 & 257.6033 & 0.005917 & 259.6926 \\
\hline 0.016719 & 299.0385 & 0.010404 & 279.4924 & 0.010479 & 282.706 \\
\hline 0.020919 & 312.7076 & 0.01603 & 300.1378 & 0.016331 & 304.3078 \\
\hline 0.025479 & 326.2354 & 0.020433 & 314.3824 & 0.020203 & 316.9206 \\
\hline 0.030769 & 339.6877 & 0.025038 & 328.4921 & 0.02504 & 331.1613 \\
\hline 0.036736 & 351.2491 & 0.030657 & 342.8275 & 0.030544 & 344.6591 \\
\hline 0.042069 & 358.7654 & 0.036636 & 354.6761 & 0.036794 & 356.2206 \\
\hline 0.047683 & 364.9721 & 0.041298 & 361.5251 & 0.041784 & 363.0235 \\
\hline 0.052957 & 369.6425 & 0.046457 & 367.6267 & 0.046831 & 368.6148 \\
\hline 0.059812 & 374.5067 & 0.051572 & 372.4652 & 0.052146 & 373.3515 \\
\hline 0.0656 & 378.0091 & 0.058062 & 377.517 & 0.058564 & 378.0707 \\
\hline 0.071589 & 381.137 & 0.065055 & 382.0243 & 0.064234 & 381.6108 \\
\hline 0.077044 & 383.6056 & 0.070193 & 384.8458 & 0.070543 & 385.0273 \\
\hline 0.08361 & 386.3465 & 0.076351 & 387.8076 & 0.076178 & 387.6555 \\
\hline 0.090134 & 388.612 & 0.082949 & 390.6082 & 0.08269 & 390.3576 \\
\hline 0.096202 & 390.6211 & 0.08782 & 392.3798 & 0.088549 & 392.6233 \\
\hline 0.101131 & 391.9727 & 0.094698 & 394.7057 & 0.095249 & 395.0327 \\
\hline 0.107473 & 393.7621 & 0.100504 & 396.5199 & 0.100906 & 396.9999 \\
\hline 0.159009 & 406.0377 & 0.107088 & 398.4475 & 0.108193 & 399.3901 \\
\hline 0.214759 & 416.211 & 0.155516 & 411.1001 & 0.157997 & 412.269 \\
\hline 0.267457 & 426.204 & 0.206498 & 422.2852 & 0.20944 & 423.9029 \\
\hline 0.318877 & 435.6107 & 0.260531 & 433.1677 & 0.26366 & 434.577 \\
\hline 0.369446 & 445.2115 & 0.31373 & 443.7086 & 0.315929 & 444.9601 \\
\hline 0.419279 & 454.3371 & 0.36323 & 453.1302 & 0.367981 & 455.2453 \\
\hline 0.471067 & 462.2606 & 0.415407 & 462.6559 & 0.418836 & 465.1319 \\
\hline 0.520583 & 470.4561 & 0.465036 & 472.4563 & 0.46851 & 475.32 \\
\hline 0.574197 & 480.3931 & 0.515019 & 482.3631 & 0.518625 & 485.3548 \\
\hline 0.62403 & 489.4941 & 0.566195 & 491.1528 & 0.568632 & 495.5241 \\
\hline 0.674678 & 499.7988 & 0.616509 & 500.7611 & 0.618762 & 506.5148 \\
\hline 0.726062 & 510.1438 & 0.668426 & 510.5314 & 0.670045 & 517.7354 \\
\hline 0.776372 & 521.5237 & 0.720128 & 519.6678 & 0.721401 & 528.6529 \\
\hline 0.825404 & 531.6139 & 0.772125 & 528.7592 & 0.770364 & 539.6442 \\
\hline 0.872119 & 544.4741 & 0.82305 & 538.3667 & 0.821596 & 551.8773 \\
\hline 0.919773 & 556.3182 & 0.871622 & 548.3598 & 0.868601 & 565.1094 \\
\hline 0.967116 & 577.359 & 0.923533 & 560.1649 & 0.917657 & 578.9857 \\
\hline 0.929125 & 558.6648 & 0.969385 & 573.4446 & 0.96443 & 597.5979 \\
\hline 0.873649 & 543.0988 & 0.925965 & 556.6053 & 0.926649 & 581.2092 \\
\hline 0.821096 & 528.6692 & 0.876025 & 542.3325 & 0.874569 & 565.4745 \\
\hline 0.769589 & 517.1929 & 0.822223 & 529.6755 & 0.82061 & 550.2737 \\
\hline 0.718991 & 505.8943 & 0.771888 & 519.1619 & 0.771872 & 538.102 \\
\hline 0.667397 & 496.5554 & 0.720819 & 509.305 & 0.721742 & 526.5015 \\
\hline 0.617485 & 487.6653 & 0.669175 & 499.0312 & 0.66919 & 515.4423 \\
\hline 0.569737 & 479.2529 & 0.620826 & 488.2794 & 0.618617 & 504.4488 \\
\hline
\end{tabular}




\begin{tabular}{|l|l|l|l|l|l|}
\hline 0.523953 & 470.9637 & 0.569706 & 481.5098 & 0.571127 & 494.1578 \\
\hline 0.472128 & 462.4533 & 0.522933 & 475.2039 & 0.522969 & 484.1916 \\
\hline 0.422562 & 454.3886 & 0.473483 & 466.1182 & 0.473549 & 474.1884 \\
\hline 0.368992 & 446.9795 & 0.421457 & 455.4327 & 0.421897 & 463.8138 \\
\hline 0.318459 & 438.7951 & 0.371482 & 446.0045 & 0.3716 & 454.1028 \\
\hline 0.267839 & 429.8966 & 0.317313 & 436.0369 & 0.320846 & 444.1382 \\
\hline 0.218396 & 418.932 & 0.268489 & 427.3233 & & \\
\hline 0.16953 & 408.4162 & 0.218226 & 418.5243 & & \\
\hline 0.12153 & 397.1053 & 0.169553 & 409.343 & & \\
\hline 0.073956 & 380.5706 & 0.122477 & 398.9382 & & \\
\hline 0.025479 & 324.8989 & 0.075035 & 383.9945 & & \\
\hline 0.01923 & 305.5477 & 0.027052 & 334.3427 & & \\
\hline
\end{tabular}

DMSO resolvated samples

Table S44. $\mathrm{N}_{2}$ isotherm data for DMSO resolvated UiO-66 samples used.

\begin{tabular}{|l|l|l|l|l|l|}
\hline $\begin{array}{l}\text { DMSO } \\
\text { resolvation 1 }\end{array}$ & $\begin{array}{l}\text { DMSO } \\
\text { resolvation 2 }\end{array}$ & $\begin{array}{l}\text { DMSO } \\
\text { resolvation 3 }\end{array}$ & \\
\hline $\mathrm{P}_{0} \mathrm{P}_{0}$ & $\begin{array}{l}\mathrm{N}_{2} \text { uptake } \\
\left(\mathrm{cc} \mathrm{g}^{-1}\right)\end{array}$ & $\mathrm{P}_{0}$ & $\begin{array}{l}\mathrm{N}_{2} \text { uptake } \\
\left(\mathrm{cc} \mathrm{g}^{-1}\right)\end{array}$ & $\mathrm{P} \mathrm{P}_{0}$ & $\begin{array}{l}\mathrm{N}_{2} \text { uptake } \\
\left(\mathrm{cc}^{-1}\right)\end{array}$ \\
\hline 0.001523 & 207.1343 & 0.003123 & 227.7704 & 0.000885 & 193.6255 \\
\hline 0.002049 & 216.5733 & 0.003605 & 233.4583 & 0.001849 & 219.1487 \\
\hline 0.002345 & 220.787 & 0.004223 & 239.0395 & 0.002125 & 223.9352 \\
\hline 0.002692 & 225.3874 & 0.004972 & 244.6233 & 0.002306 & 226.8072 \\
\hline 0.003103 & 230.1531 & 0.005821 & 250.2694 & 0.002545 & 229.9729 \\
\hline 0.003601 & 235.2533 & 0.006828 & 256.0483 & 0.002814 & 233.3198 \\
\hline 0.004185 & 240.5042 & 0.007878 & 261.462 & 0.003147 & 237.0847 \\
\hline 0.004842 & 245.8117 & 0.008958 & 266.6134 & 0.003546 & 240.9564 \\
\hline 0.0056 & 251.1342 & 0.010044 & 271.3682 & 0.004336 & 247.9458 \\
\hline 0.010268 & 275.214 & 0.011238 & 276.2166 & 0.005294 & 254.9943 \\
\hline 0.015774 & 295.8163 & 0.014541 & 288.4471 & 0.012117 & 288.3554 \\
\hline 0.020067 & 309.7182 & 0.018181 & 300.7328 & 0.016127 & 302.9117 \\
\hline 0.026554 & 329.2481 & 0.022131 & 313.601 & 0.020812 & 317.8525 \\
\hline 0.031223 & 340.6304 & 0.02657 & 327.0567 & 0.025946 & 332.7246 \\
\hline 0.035105 & 348.0743 & 0.031312 & 339.4847 & 0.030087 & 342.5404 \\
\hline 0.041592 & 357.8152 & 0.036953 & 351.2447 & 0.037106 & 355.0728 \\
\hline 0.046434 & 363.1946 & 0.043601 & 361.6952 & 0.041029 & 360.2448 \\
\hline 0.052741 & 368.8085 & 0.049644 & 369.1616 & 0.045409 & 365.0378 \\
\hline 0.059062 & 373.2672 & 0.055565 & 375.035 & 0.050232 & 369.3914 \\
\hline 0.064972 & 376.7843 & 0.062379 & 380.9115 & 0.056243 & 373.7512 \\
\hline 0.070809 & 379.7543 & 0.067451 & 384.6971 & 0.061377 & 376.9561 \\
\hline 0.076178 & 382.1206 & 0.074027 & 389.0684 & 0.067802 & 380.3461 \\
\hline 0.082759 & 384.6424 & 0.079539 & 392.1782 & 0.073988 & 383.1308 \\
\hline 0.089239 & 386.7802 & 0.084273 & 394.7556 & 0.07984 & 385.4756 \\
\hline & & & & & \\
\hline
\end{tabular}




\begin{tabular}{|c|c|c|c|c|c|}
\hline 0.095156 & 388.5695 & 0.090633 & 397.892 & 0.085532 & 387.5155 \\
\hline 0.103281 & 390.7803 & 0.097094 & 400.7993 & 0.092494 & 389.8582 \\
\hline 0.108671 & 392.0785 & 0.102339 & 402.9532 & 0.098295 & 391.6611 \\
\hline 0.157718 & 402.865 & 0.109527 & 405.9964 & 0.10567 & 393.7766 \\
\hline 0.208633 & 412.0988 & 0.161027 & 427.0103 & 0.154081 & 404.7014 \\
\hline 0.262723 & 420.9548 & 0.214902 & 447.5239 & 0.207098 & 414.2582 \\
\hline 0.315919 & 429.5759 & 0.265719 & 466.5928 & 0.260991 & 422.7812 \\
\hline 0.36809 & 437.5547 & 0.316356 & 485.152 & 0.313232 & 430.834 \\
\hline 0.418435 & 444.7409 & 0.365784 & 503.3248 & 0.363021 & 438.2206 \\
\hline 0.470216 & 452.9029 & 0.417112 & 522.3699 & 0.413985 & 445.3846 \\
\hline 0.52129 & 460.913 & 0.468799 & 541.8887 & 0.465261 & 453.1067 \\
\hline 0.57157 & 467.7585 & 0.521062 & 562.3364 & 0.517298 & 461.0641 \\
\hline 0.620249 & 475.7116 & 0.569915 & 580.2299 & 0.568436 & 468.2938 \\
\hline 0.671922 & 484.3409 & 0.621157 & 598.1553 & 0.618943 & 476.2239 \\
\hline 0.723097 & 493.0775 & 0.673808 & 617.2155 & 0.670234 & 484.2956 \\
\hline 0.772699 & 501.6379 & 0.726668 & 635.597 & 0.72151 & 492.3939 \\
\hline 0.823852 & 511.2097 & 0.77655 & 652.3803 & 0.770683 & 500.1715 \\
\hline 0.871657 & 520.9002 & 0.826158 & 670.6594 & 0.819101 & 508.2822 \\
\hline 0.919982 & 532.1971 & 0.875601 & 689.2794 & 0.866984 & 517.1739 \\
\hline 0.964439 & 548.297 & 0.926173 & 709.2095 & 0.914829 & 526.9179 \\
\hline 0.928324 & 534.1407 & 0.975285 & 725.9282 & 0.960493 & 540.1003 \\
\hline 0.87577 & 520.275 & 0.927037 & 698.7527 & 0.924612 & 528.8583 \\
\hline 0.822265 & 508.8952 & 0.873083 & 671.5869 & 0.875577 & 518.1628 \\
\hline 0.772843 & 499.4699 & 0.82082 & 648.5256 & 0.82256 & 508.1928 \\
\hline 0.719727 & 490.3001 & 0.770031 & 628.0054 & 0.773228 & 499.5125 \\
\hline 0.669107 & 482.8346 & 0.719898 & 608.9294 & 0.72222 & 491.2537 \\
\hline 0.618019 & 475.0268 & 0.6687 & 588.2436 & 0.671249 & 483.3461 \\
\hline 0.570134 & 467.7274 & 0.622193 & 565.6982 & 0.62167 & 475.4395 \\
\hline 0.521528 & 461.0072 & 0.568411 & 555.8337 & 0.571279 & 467.6092 \\
\hline 0.471688 & 453.0673 & 0.521141 & 541.7245 & 0.522947 & 460.7098 \\
\hline 0.422714 & 445.2969 & 0.472886 & 523.1293 & 0.472788 & 452.9844 \\
\hline 0.371596 & 439.8958 & 0.418745 & 501.7958 & 0.419917 & 445.122 \\
\hline 0.319786 & 432.8837 & 0.369504 & 482.8197 & 0.371672 & 438.3586 \\
\hline 0.270848 & 425.1633 & 0.316169 & 464.2354 & 0.319577 & 430.7284 \\
\hline 0.219017 & 414.9917 & 0.279648 & 452.4936 & & \\
\hline 0.170165 & 405.4757 & 0.218996 & 433.6161 & & \\
\hline 0.122374 & 394.9524 & 0.169128 & 417.8026 & & \\
\hline 0.075218 & 380.3479 & 0.120657 & 401.2111 & & \\
\hline 0.02776 & 331.7619 & 0.070912 & 379.0206 & & \\
\hline 0.019461 & 307.0171 & & & & \\
\hline
\end{tabular}




\section{$\mathrm{H}_{2} \mathrm{O}$ resolvated samples}

Table S45. $\mathrm{N}_{2}$ isotherm data for $\mathrm{H}_{2} \mathrm{O}$ resolvated UiO-66 samples used.

\begin{tabular}{|c|c|c|c|c|c|}
\hline $\begin{array}{l}\mathrm{H}_{2} \mathrm{O} \\
\text { resolvation } 1\end{array}$ & & $\begin{array}{l}\mathrm{H}_{2} \mathrm{O} \\
\text { resolvation } 2\end{array}$ & & $\begin{array}{l}\mathrm{H}_{2} \mathrm{O} \\
\text { resolvation } 3\end{array}$ & \\
\hline $\mathrm{P} / \mathrm{P}_{0}$ & $\begin{array}{l}\mathrm{N}_{2} \text { uptake } \\
\left(\mathrm{cc} \mathrm{g}^{-1}\right)\end{array}$ & $\mathrm{P} / \mathrm{P}_{0}$ & $\begin{array}{l}\mathrm{N}_{2} \text { uptake } \\
\left(\mathrm{cc} \mathrm{g}^{-1}\right)\end{array}$ & $\mathrm{P} / \mathrm{P}_{0}$ & $\begin{array}{l}\mathrm{N}_{2} \text { uptake } \\
\left(\mathrm{cc} \mathrm{g}^{-1}\right)\end{array}$ \\
\hline 0.001616 & 201.3387 & 0.001223 & 199.4368 & 0.001218 & 203.8998 \\
\hline 0.002129 & 209.7235 & 0.001554 & 207.5004 & 0.001726 & 216.0363 \\
\hline 0.002497 & 214.696 & 0.002058 & 215.7701 & 0.002052 & 222.1101 \\
\hline 0.002973 & 220.0083 & 0.002338 & 219.6397 & 0.002277 & 225.6725 \\
\hline 0.003492 & 225.4698 & 0.002684 & 223.5668 & 0.002589 & 229.6124 \\
\hline 0.004142 & 231.1886 & 0.003065 & 227.8922 & 0.002944 & 233.7099 \\
\hline 0.0049 & 236.9697 & 0.003554 & 232.2803 & 0.003336 & 237.9555 \\
\hline 0.005765 & 242.6751 & 0.004079 & 236.8058 & 0.003836 & 242.6536 \\
\hline 0.00674 & 248.2531 & 0.004727 & 241.5937 & 0.004416 & 247.5124 \\
\hline 0.010622 & 266.2654 & 0.005439 & 246.2804 & 0.005054 & 252.3962 \\
\hline 0.015586 & 283.9461 & 0.011526 & 274.7234 & 0.010544 & 281.4232 \\
\hline 0.020847 & 300.2849 & 0.01549 & 288.4733 & 0.015359 & 299.4306 \\
\hline 0.026042 & 314.7145 & 0.021318 & 306.6858 & 0.020892 & 317.3767 \\
\hline 0.030191 & 324.2175 & 0.025182 & 317.5815 & 0.02504 & 329.5601 \\
\hline 0.036772 & 335.6971 & 0.030528 & 329.9337 & 0.030159 & 342.0033 \\
\hline 0.042018 & 342.3491 & 0.035988 & 339.5846 & 0.035794 & 352.6853 \\
\hline 0.047444 & 347.6389 & 0.040701 & 345.8811 & 0.041755 & 361.0192 \\
\hline 0.052871 & 351.95 & 0.045766 & 351.2531 & 0.046722 & 366.3131 \\
\hline 0.060015 & 356.4669 & 0.051069 & 355.6942 & 0.051791 & 370.7837 \\
\hline 0.065722 & 359.4879 & 0.057925 & 360.2344 & 0.057425 & 374.9184 \\
\hline 0.071762 & 362.2177 & 0.064055 & 363.5791 & 0.063284 & 378.4846 \\
\hline 0.077246 & 364.3908 & 0.070488 & 366.5337 & 0.069673 & 381.8441 \\
\hline 0.083899 & 366.7331 & 0.076373 & 368.8234 & 0.07567 & 384.5499 \\
\hline 0.09004 & 368.6515 & 0.081481 & 370.526 & 0.082262 & 387.2167 \\
\hline 0.097184 & 370.6828 & 0.08705 & 372.1657 & 0.08728 & 389.1247 \\
\hline 0.102054 & 371.8865 & 0.093878 & 374.0556 & 0.095663 & 392.0544 \\
\hline 0.107827 & 373.2769 & 0.102339 & 376.1203 & 0.102907 & 394.3989 \\
\hline 0.161867 & 384.909 & 0.108886 & 377.5991 & 0.108672 & 396.1669 \\
\hline 0.2153 & 395.1966 & 0.160228 & 387.1772 & 0.156837 & 407.924 \\
\hline 0.268799 & 404.9267 & 0.211599 & 394.6783 & 0.207308 & 418.0084 \\
\hline 0.319837 & 412.9857 & 0.264085 & 401.3731 & 0.259563 & 427.5638 \\
\hline 0.369634 & 420.961 & 0.318514 & 407.6257 & 0.31176 & 436.7131 \\
\hline 0.421292 & 429.088 & 0.369094 & 413.1968 & 0.363021 & 445.4721 \\
\hline 0.472366 & 436.6736 & 0.420947 & 418.7279 & 0.415718 & 454.4455 \\
\hline 0.522026 & 444.149 & 0.472368 & 424.0642 & 0.46761 & 463.1895 \\
\hline 0.574962 & 451.9511 & 0.524185 & 429.0313 & 0.518886 & 472.3494 \\
\hline 0.625805 & 460.0464 & 0.5758 & 434.1315 & 0.567167 & 480.9253 \\
\hline
\end{tabular}




\begin{tabular}{|l|l|l|l|l|l|}
\hline 0.676367 & 468.4515 & 0.626625 & 438.7568 & 0.617573 & 489.6348 \\
\hline 0.726055 & 476.3277 & 0.679096 & 442.9372 & 0.670082 & 499.2787 \\
\hline 0.774928 & 486.449 & 0.730849 & 446.9914 & 0.722249 & 508.9332 \\
\hline 0.826067 & 496.0831 & 0.780637 & 450.8806 & 0.771248 & 518.1093 \\
\hline 0.877733 & 505.8923 & 0.831518 & 455.3908 & 0.822495 & 528.1791 \\
\hline 0.926686 & 517.2628 & 0.879774 & 460.096 & 0.870283 & 538.2521 \\
\hline 0.970602 & 539.0277 & 0.927389 & 466.2547 & 0.919688 & 549.7045 \\
\hline 0.927847 & 516.7437 & 0.973537 & 474.0941 & 0.965373 & 565.1727 \\
\hline 0.87344 & 502.6831 & 0.918842 & 460.756 & 0.924459 & 550.6321 \\
\hline 0.822084 & 492.4342 & 0.872816 & 453.0896 & 0.872502 & 537.9214 \\
\hline 0.770079 & 482.1671 & 0.821978 & 446.7852 & 0.820341 & 526.4641 \\
\hline 0.717988 & 471.6027 & 0.770945 & 441.8425 & 0.771676 & 516.6518 \\
\hline 0.669411 & 465.2086 & 0.719761 & 436.7765 & 0.721909 & 507.1537 \\
\hline 0.617788 & 457.806 & 0.669937 & 431.4301 & 0.669552 & 497.4564 \\
\hline 0.56986 & 450.4789 & 0.619185 & 427.31 & 0.62196 & 488.4761 \\
\hline 0.520872 & 443.201 & 0.567526 & 424.5767 & 0.571525 & 479.8475 \\
\hline 0.46987 & 435.2778 & 0.521098 & 420.8727 & 0.52323 & 471.1393 \\
\hline 0.419892 & 429.1885 & 0.472677 & 416.0114 & 0.473905 & 462.3363 \\
\hline 0.371012 & 423.2674 & 0.420731 & 409.9564 & 0.419714 & 453.3865 \\
\hline 0.31729 & 415.5065 & 0.370885 & 404.6518 & 0.368554 & 444.6458 \\
\hline 0.266576 & 406.8402 & 0.318939 & 399.4781 & 0.318177 & 436.0436 \\
\hline 0.218021 & 396.1763 & 0.267697 & 394.6431 & & \\
\hline 0.169342 & 385.9977 & 0.219118 & 389.7131 & & \\
\hline 0.12 & 375.1064 & 0.169618 & 383.8973 & & \\
\hline 0.073306 & 360.6757 & 0.121376 & 376.6235 & & \\
\hline 0.029094 & 320.1844 & 0.075135 & 365.2761 & & \\
\hline 0.01874 & 291.9583 & & & & \\
\hline & & & & & \\
\hline
\end{tabular}

\section{DMF RSE samples}

Table S46. $\mathrm{N}_{2}$ isotherm data for DMF RSE UiO-66 samples used.

\begin{tabular}{|l|l|l|l|l|l|}
\hline DMF RSE 1 & & DMF RSE 2 & & DMF RSE 3 & \\
\hline $\mathrm{P} / \mathrm{P}_{0}$ & $\begin{array}{l}\mathrm{N}_{2} \text { uptake } \\
\left(\mathrm{cc} \mathrm{g}^{-1}\right)\end{array}$ & $\mathrm{P} / \mathrm{P}_{0}$ & $\begin{array}{l}\mathrm{N}_{2} \text { uptake } \\
\left(\mathrm{cc} \mathrm{g}^{-1}\right)\end{array}$ & $\mathrm{P} / \mathrm{P}_{0}$ & $\begin{array}{l}\mathrm{N}_{2} \text { uptake } \\
\left(\mathrm{cc} \mathrm{g}^{-1}\right)\end{array}$ \\
\hline 0.002288 & 217.1789 & 0.001614 & 212.3994 & 0.00125 & 204.1998 \\
\hline 0.002638 & 222.0843 & 0.002047 & 219.7736 & 0.001561 & 212.0072 \\
\hline 0.003089 & 227.4048 & 0.002253 & 223.0132 & 0.002197 & 222.8863 \\
\hline 0.003604 & 232.8919 & 0.00251 & 226.4157 & 0.002426 & 226.2812 \\
\hline 0.004233 & 238.729 & 0.002767 & 230.1167 & 0.002722 & 230.101 \\
\hline 0.004969 & 244.5807 & 0.003082 & 234.0856 & 0.003062 & 234.2159 \\
\hline 0.005756 & 250.4795 & 0.003471 & 238.4315 & 0.003462 & 238.5032 \\
\hline 0.006657 & 256.2727 & 0.004337 & 246.825 & 0.003928 & 243.1313 \\
\hline 0.007665 & 262.0558 & 0.005342 & 255.0011 & 0.004468 & 247.8759 \\
\hline 0.010031 & 273.4788 & 0.010398 & 282.7042 & 0.005089 & 252.7408 \\
\hline
\end{tabular}




\begin{tabular}{|c|c|c|c|c|c|}
\hline 0.015201 & 292.5409 & 0.015366 & 301.6552 & 0.010896 & 283.4783 \\
\hline 0.020528 & 308.6212 & 0.020305 & 318.1811 & 0.016022 & 302.3424 \\
\hline 0.025826 & 322.9442 & 0.026051 & 335.2045 & 0.021312 & 319.404 \\
\hline 0.031874 & 338.0135 & 0.030732 & 346.4949 & 0.025321 & 331.3118 \\
\hline 0.036279 & 347.838 & 0.035957 & 356.1729 & 0.030136 & 343.9457 \\
\hline 0.040948 & 357.9423 & 0.041013 & 363.3188 & 0.035581 & 355.3486 \\
\hline 0.045524 & 366.7299 & 0.045284 & 367.9535 & 0.041047 & 364.1366 \\
\hline 0.051215 & 375.7571 & 0.050252 & 372.3048 & 0.045271 & 369.3745 \\
\hline 0.057528 & 383.7859 & 0.055477 & 376.0177 & 0.053135 & 376.5072 \\
\hline 0.063013 & 389.6464 & 0.061414 & 379.5785 & 0.057847 & 379.6609 \\
\hline 0.06899 & 394.9872 & 0.067534 & 382.6012 & 0.063483 & 382.8478 \\
\hline 0.074788 & 399.3221 & 0.072817 & 384.9015 & & 385.139 \\
\hline 0.081595 & 403.768 & 0.079715 & 387.4132 & & 387.4524 \\
\hline 0.08 & 407.2057 & 0.08 & 5274 & & 389.3438 \\
\hline 0.09 & 410.2594 & $0.0 \varsigma$ & 0443 & & 391.791 \\
\hline 0.10 & 412.4781 & $0.0 \varsigma$ & 392.841 & & 393.328 \\
\hline 0.106927 & 414.9526 & 0.10 & 394.0244 & 711 & 395.2537 \\
\hline 0.156991 & 430.3254 & 0.150287 & 402.6848 & 0.1 & 396.0818 \\
\hline 0.211524 & 443.908 & 0.198279 & 408.6308 & 0483 & 406.2414 \\
\hline 0.265356 & 456.782 & 0.246395 & 413.4043 & 0.200022 & 413.3226 \\
\hline 0.316514 & 468.2912 & 0.296317 & 418.0487 & 0.247454 & 419.0163 \\
\hline 0.366099 & 479.9908 & 0.347171 & 421.8038 & 0.29524 & 424.3148 \\
\hline 0.416384 & 491.8514 & 0.395728 & 425.8807 & 0.355365 & 429.614 \\
\hline 0.467721 & 504.3882 & 0.451711 & 429.5751 & 0.399623 & 433.615 \\
\hline 0.518328 & 515.9221 & 0.500628 & 432.673 & 0.445604 & 438.7047 \\
\hline 0.570673 & 529.6899 & 0.545435 & 436.0053 & 0.495129 & 442.5089 \\
\hline 0.61885 & 541.6147 & 0.599774 & 440.4693 & 0.550587 & 447.6832 \\
\hline 0.670601 & 554.4229 & 0.649505 & 443.2194 & 0.595555 & 450.2192 \\
\hline 0.722002 & 567.792 & 0.696235 & 447.4365 & 0.647417 & 454.8773 \\
\hline 0.773989 & 581.8063 & 0.749944 & 451.2204 & 0.699124 & 458.3985 \\
\hline 0.824089 & 595.5532 & 0.802992 & 455.0473 & 0.746037 & 463.3424 \\
\hline 0.873953 & 611.1694 & 0.845905 & 459.8853 & 0.796657 & 466.7771 \\
\hline 0.921385 & 628.3661 & 0.898513 & 466.1537 & 0.854015 & 473.2985 \\
\hline 0.968461 & 655.1918 & 0.94638 & 473.8162 & 0.896749 & 478.5074 \\
\hline 0.928964 & 630.196 & 0.924556 & 470.8493 & 0.951704 & 489.4335 \\
\hline 0.874632 & 607.5441 & 0.868867 & 464.9855 & 0.925732 & 484.736 \\
\hline 0.822788 & 589.5834 & 0.816963 & 461.1154 & 0.869224 & 477.4908 \\
\hline 0.770522 & 573.6094 & 0.77697 & 458.7807 & 0.827481 & 474.4899 \\
\hline 0.720107 & 558.9412 & 0.729213 & 456.3099 & 0.766106 & 470.0291 \\
\hline 0.671051 & 546.2081 & 0.664813 & 451.8745 & 0.716862 & 465.6952 \\
\hline 0.622189 & 534.4182 & 0.628922 & 448.8728 & 0.665577 & 461.6121 \\
\hline 0.572232 & 522.2364 & 0.568748 & 445.674 & 0.627082 & 461.0792 \\
\hline 0.52259 & 509.729 & 0.517564 & 441.572 & 0.570078 & 456.4354 \\
\hline
\end{tabular}




\begin{tabular}{|l|l|l|l|l|l|}
\hline 0.472812 & 498.5951 & 0.471429 & 438.0162 & 0.516152 & 451.5152 \\
\hline 0.421268 & 487.162 & 0.414712 & 434.062 & & \\
\hline 0.367815 & 475.3783 & 0.364548 & 429.6603 & & \\
\hline 0.31828 & 464.3036 & 0.31624 & 425.6864 & & \\
\hline 0.26891 & 454.0433 & 0.267933 & 420.4925 & & \\
\hline 0.218109 & 442.7047 & 0.220506 & 415.5875 & & \\
\hline 0.168903 & 431.0345 & 0.175354 & 409.4443 & & \\
\hline 0.121428 & 417.8354 & 0.123363 & 400.4988 & & \\
\hline 0.075167 & 397.991 & 0.075701 & 387.1375 & & \\
\hline 0.027506 & 327.9935 & 0.029213 & 344.252 & & \\
\hline 0.019634 & 306.1631 & 0.01938 & 315.5485 & & \\
\hline
\end{tabular}

\section{DMSO RSE samples}

Table S47. $\mathrm{N}_{2}$ isotherm data for DMSO RSE UiO-66 samples used.

\begin{tabular}{|l|l|l|l|l|l|}
\hline DMSO RSE 1 & & DMSO RSE 2 & & DMSO RSE 3 & \\
\hline $\mathrm{P} / \mathrm{P}_{0}$ & $\begin{array}{l}\mathrm{N}_{2} \text { uptake } \\
\left(\mathrm{cc} \mathrm{g}^{-1}\right)\end{array}$ & $\mathrm{P} / \mathrm{P}_{0}$ & $\begin{array}{l}\mathrm{N}_{2} \text { uptake } \\
\left(\mathrm{cc} \mathrm{g}^{-1}\right)\end{array}$ & $\mathrm{P} / \mathrm{P}_{0}$ & $\begin{array}{l}\mathrm{N}_{2} \text { uptake } \\
\left(\mathrm{cc} \mathrm{g}^{-1}\right)\end{array}$ \\
\hline 0.00216 & 210.547 & 0.001504 & 204.6585 & 0.000999 & 194.4961 \\
\hline 0.002584 & 216.1561 & 0.002011 & 214.3003 & 0.00159 & 209.143 \\
\hline 0.003051 & 222.2265 & 0.002157 & 216.8747 & 0.002034 & 217.0137 \\
\hline 0.003598 & 228.5204 & 0.002363 & 219.6239 & 0.002241 & 220.338 \\
\hline 0.004211 & 235.0291 & 0.002576 & 222.6181 & 0.002515 & 224.1095 \\
\hline 0.005014 & 242.0073 & 0.002825 & 225.8539 & 0.002855 & 228.1488 \\
\hline 0.00586 & 248.6573 & 0.003104 & 229.4381 & 0.00321 & 232.3873 \\
\hline 0.006714 & 255.2849 & 0.004418 & 242.6841 & 0.003662 & 236.965 \\
\hline 0.007846 & 262.3035 & 0.005232 & 249.2606 & 0.004172 & 241.65 \\
\hline 0.010108 & 273.5397 & 0.010655 & 278.1803 & 0.005311 & 250.6617 \\
\hline 0.016209 & 295.8638 & 0.016306 & 299.0605 & 0.011281 & 280.699 \\
\hline 0.02188 & 312.5927 & 0.02029 & 311.8166 & 0.016193 & 298.0977 \\
\hline 0.02539 & 322.1657 & 0.026197 & 329.3159 & 0.021541 & 315.0666 \\
\hline 0.031638 & 338.1394 & 0.030057 & 338.7501 & 0.026172 & 328.4817 \\
\hline 0.035754 & 347.7475 & 0.036361 & 351.0424 & 0.030151 & 338.3587 \\
\hline 0.040483 & 357.913 & 0.040507 & 357.1241 & 0.035581 & 349.2935 \\
\hline 0.045322 & 366.9672 & 0.045739 & 363.1375 & 0.041062 & 357.8551 \\
\hline 0.051357 & 375.7078 & 0.052072 & 368.6691 & 0.045271 & 362.873 \\
\hline 0.057846 & 383.1324 & 0.056402 & 371.7128 & 0.050006 & 367.2479 \\
\hline 0.062495 & 387.7696 & 0.061318 & 374.6357 & 0.055443 & 371.0991 \\
\hline 0.068377 & 392.3449 & 0.066015 & 377.1134 & 0.061715 & 374.8752 \\
\hline 0.073799 & 396.1018 & 0.071181 & 379.429 & 0.066923 & 377.4894 \\
\hline 0.079806 & 399.3439 & 0.078123 & 382.1291 & 0.073218 & 380.0789 \\
\hline 0.086608 & 402.3589 & 0.08436 & 384.1993 & 0.078004 & 381.8494 \\
\hline 0.092614 & 405.0554 & 0.089834 & 385.8336 & 0.084862 & 383.9268 \\
\hline 0.099161 & 407.3483 & 0.096336 & 387.6314 & 0.090698 & 385.8111 \\
\hline & & & & & \\
\hline
\end{tabular}




\begin{tabular}{|l|l|l|l|l|l|}
\hline 0.103875 & 408.9085 & 0.102566 & 389.1885 & 0.097503 & 387.5745 \\
\hline 0.148146 & 419.7028 & 0.147814 & 397.2725 & 0.103873 & 389.0125 \\
\hline 0.195512 & 428.5284 & 0.200979 & 403.6902 & 0.146207 & 397.2727 \\
\hline 0.245592 & 435.6804 & 0.24889 & 408.3095 & 0.200925 & 404.9095 \\
\hline 0.296738 & 442.1317 & 0.295326 & 412.3516 & 0.245959 & 409.8343 \\
\hline 0.345242 & 449.6349 & 0.345659 & 416.4348 & 0.298569 & 414.9604 \\
\hline 0.396278 & 455.6088 & 0.400344 & 420.6974 & 0.346 & 419.5496 \\
\hline 0.448526 & 460.7012 & 0.449253 & 424.0052 & 0.396361 & 424.013 \\
\hline 0.499168 & 465.6067 & 0.495131 & 427.5629 & 0.447542 & 427.4713 \\
\hline 0.546701 & 470.9648 & 0.551393 & 431.4914 & 0.503185 & 431.7129 \\
\hline 0.599679 & 476.7263 & 0.599055 & 434.0463 & 0.549048 & 434.8965 \\
\hline 0.650832 & 482.1131 & 0.645704 & 437.6612 & 0.597034 & 438.8575 \\
\hline 0.698993 & 485.4662 & 0.698796 & 441.709 & 0.648386 & 442.1209 \\
\hline 0.750708 & 489.3412 & 0.746311 & 445.2055 & 0.696727 & 446.3196 \\
\hline 0.795906 & 495.2897 & 0.801098 & 449.3354 & 0.748449 & 449.7525 \\
\hline 0.848533 & 499.7098 & 0.845994 & 453.3471 & 0.799009 & 454.1377 \\
\hline 0.898957 & 502.3092 & 0.90092 & 459.1652 & 0.849074 & 458.1292 \\
\hline 0.947256 & 518.4755 & 0.946439 & 466.4926 & 0.901617 & 466.2842 \\
\hline 0.926763 & 511.5555 & 0.927837 & 465.1625 & 0.949729 & 474.8132 \\
\hline 0.867925 & 507.7288 & 0.87743 & 459.9633 & 0.927781 & 470.7636 \\
\hline 0.829222 & 503.9205 & 0.821469 & 454.0166 & 0.867256 & 464.3081 \\
\hline 0.764889 & 498.8694 & 0.765148 & 451.268 & 0.829013 & 460.5128 \\
\hline 0.727383 & 501.2963 & 0.728068 & 448.5528 & 0.769213 & 456.2076 \\
\hline 0.669523 & 496.0455 & 0.66744 & 444.7434 & 0.728654 & 454.6698 \\
\hline 0.617428 & 492.6927 & 0.62912 & 442.1659 & 0.667227 & 451.1119 \\
\hline 0.567224 & 489.5302 & 0.569453 & 438.0171 & 0.616763 & 447.3042 \\
\hline 0.519523 & 483.5341 & 0.51774 & 434.8219 & 0.569161 & 443.7685 \\
\hline 0.473873 & 477.463 & 0.469506 & 431.4933 & & \\
\hline 0.428967 & 473.9998 & 0.429858 & 428.0812 & & \\
\hline 0.364407 & 465.4443 & 0.369105 & 423.3039 & & \\
\hline 0.329193 & 460.345 & 0.318706 & 418.6982 & & \\
\hline 0.268757 & 450.3834 & 0.268762 & 414.3331 & & \\
\hline 0.218815 & 441.3949 & 0.220778 & 409.1544 & & \\
\hline 0.171858 & 431.6461 & 0.17564 & 403.408 & & \\
\hline 0.11899 & 417.5945 & 0.12508 & 395.3986 & & \\
\hline 0.071574 & 396.4043 & 0.077961 & 382.9888 & & \\
\hline 0.028843 & 331.7714 & 0.029089 & 337.5387 & & \\
\hline 0.017428 & 296.7115 & 0.019138 & 308.548 & & \\
\hline & & & & & \\
\hline
\end{tabular}




\section{$\mathrm{H}_{2} \mathrm{O}$ RSE samples}

Table S48. $\mathrm{N}_{2}$ isotherm data for $\mathrm{H}_{2} \mathrm{O}$ RSE UiO-66 samples used.

\begin{tabular}{|c|c|c|c|c|c|}
\hline $\mathrm{H}_{2} \mathrm{O}$ RSE 1 & & $\mathrm{H}_{2} \mathrm{O} \mathrm{RSE} 2$ & & $\mathrm{H}_{2} \mathrm{O} \mathrm{RSE} 3$ & \\
\hline$P / P_{0}$ & $\begin{array}{l}\mathrm{N}_{2} \text { uptake } \\
\left(\mathrm{cc} \mathrm{g}^{-1}\right)\end{array}$ & $\mathrm{P} / \mathrm{P}_{0}$ & $\begin{array}{l}\mathrm{N}_{2} \text { uptake } \\
\left(\mathrm{cc} \mathrm{g}^{-1}\right)\end{array}$ & $\mathrm{P} / \mathrm{P}_{0}$ & $\begin{array}{l}\mathrm{N}_{2} \text { uptake } \\
\left(\mathrm{cc} \mathrm{g}^{-1}\right)\end{array}$ \\
\hline 0.002545 & 175.1699 & 0.001651 & 205.094 & 0.000851 & 194.0369 \\
\hline 0.003175 & 182.4433 & 0.002121 & 213.2436 & 0.001857 & 218.2992 \\
\hline 0.003975 & 189.9229 & 0.002334 & 216.6383 & 0.002049 & 222.1557 \\
\hline 0.004876 & 197.0922 & 0.002605 & 220.2342 & 0.002204 & 224.3693 \\
\hline 0.005913 & 204.0872 & 0.002928 & 224.1131 & 0.002382 & 226.92 \\
\hline 0.007071 & 210.6729 & .003288 & 228.2514 & 0.002589 & 229.6962 \\
\hline 0.008251 & 217.0108 & 0.00372 & 232.7804 & 0.002826 & 232.6347 \\
\hline 0.009509 & 222.8304 & 0.004227 & 237.4573 & 0.003099 & 235.8622 \\
\hline 0.010832 & 228.3076 & .005328 & 246.2561 & 0.004239 & 247.2045 \\
\hline 0.014178 & 240.5482 & 0.011029 & 275.6219 & 0.005178 & 254.6003 \\
\hline 0.018032 & 252.7786 & 0.015858 & 293.1683 & 0.010127 & .9145 \\
\hline 0.022665 & 265.7282 & .021163 & 309.8397 & 0.015164 & 341 \\
\hline 0.027463 & 277.7 & 025354 & 321.4332 & & 3253 \\
\hline 0.032854 & 289.5397 & 0.030622 & 333.3431 & 0.025047 & 7043 \\
\hline 0.038717 & 515 & .035972 & 342.5356 & 0.03004 & 3516 \\
\hline & & & & & 5984 \\
\hline 0.049914 & 617 & 0.046656 & 354.5772 & 040323 & 449 \\
\hline 0.056191 & 325.7 & 0.050839 & 357.8636 & 0.046359 & 2989 \\
\hline 0.062505 & 332. & .056402 & 361.4583 & 052417 & 2408 \\
\hline 0.06766 & 051 & 06214 & 364.6052 & 056471 & 8115 \\
\hline 0.073144 & 341. & 0.068003 & 367.3565 & 0.061745 & 7863 \\
\hline 0.078263 & 345. & 0.07336 & 369.4378 & 0.066753 & 382.2262 \\
\hline 0.084777 & 97 & 80508 & 372.0061 & 072323 & 384.5838 \\
\hline 0.090776 & 489 & 086429 & 373.847 & 0.077006 & \\
\hline 0.097154 & 356.2961 & 0.090289 & 374.9774 & 0.083441 & 388 \\
\hline 0.104261 & 359.7 & .09682 & 376.7096 & 0.088479 & 6884 \\
\hline 0.109637 & 362.0 & 0.102302 & 378.0835 & 0.095477 & 4228 \\
\hline 0.160874 & 382.8919 & 0.149509 & 386.5029 & 0.101823 & 392.9903 \\
\hline 0.212675 & 401.4724 & 0.20219 & 393.2482 & 0.147036 & 400.7622 \\
\hline 0.266858 & 421.1277 & 0.245368 & 397.3567 & 0.197944 & 562 \\
\hline 0.319145 & 440.2717 & 0.298335 & 402.1574 & 0.246869 & 410.8801 \\
\hline 0.370239 & 459.5949 & 0.34519 & 406.1527 & 0.298436 & 415.4457 \\
\hline 0.420217 & 478.6945 & 0.397262 & 410.1606 & 0.347376 & 419.0683 \\
\hline 0.471732 & 498.2328 & 0.448093 & 413.9706 & 0.395281 & 421.5043 \\
\hline 0.52284 & 519.5067 & 0.497524 & 417.0231 & 0.445508 & 424.4807 \\
\hline 0.571331 & 538.3366 & 0.552303 & 421.0888 & 0.503074 & 427.9679 \\
\hline 0.622975 & 557.4315 & 0.597507 & 424.8589 & 0.547598 & 430.5444 \\
\hline 0.67369 & 578.8417 & 0.645734 & 427.6534 & 0.596812 & 432.2061 \\
\hline
\end{tabular}




\begin{tabular}{|l|l|l|l|l|l|}
\hline 0.724905 & 601.3077 & 0.69782 & 430.1858 & 0.652906 & 435.2099 \\
\hline 0.775762 & 621.7194 & 0.751294 & 432.666 & 0.697829 & 437.7748 \\
\hline 0.825111 & 644.3082 & 0.796857 & 436.8188 & 0.749107 & 440.1714 \\
\hline 0.873145 & 666.6182 & 0.852025 & 439.7418 & 0.797478 & 444.2521 \\
\hline 0.923059 & 692.0793 & 0.900127 & 444.8408 & 0.850642 & 448.4073 \\
\hline 0.970377 & 720.0563 & 0.94729 & 452.5811 & 0.900648 & 452.188 \\
\hline 0.926154 & 690.7042 & 0.927572 & 449.2316 & 0.946503 & 459.6793 \\
\hline 0.87391 & 659.9841 & 0.869013 & 445.7231 & 0.92929 & 457.7776 \\
\hline 0.819792 & 633.4317 & 0.820669 & 442.8323 & 0.87333 & 451.2028 \\
\hline 0.7706 & 608.3919 & 0.779906 & 441.095 & 0.821763 & 447.675 \\
\hline 0.721037 & 584.8852 & 0.72015 & 439.1412 & 0.764249 & 445.2909 \\
\hline 0.670844 & 563.026 & 0.678785 & 435.8444 & 0.727574 & 444.4219 \\
\hline 0.619014 & 542.0896 & 0.619382 & 433.5869 & 0.669209 & 441.0631 \\
\hline 0.571395 & 524.8843 & 0.565974 & 430.2443 & 0.618042 & 438.0937 \\
\hline 0.521546 & 506.4053 & 0.516926 & 425.9859 & 0.578718 & 437.1727 \\
\hline 0.471446 & 486.8114 & 0.469235 & 422.6419 & & \\
\hline 0.417993 & 468.0119 & 0.427906 & 420.0932 & & \\
\hline 0.36961 & 451.1666 & 0.368555 & 414.5163 & & \\
\hline 0.320539 & 433.8956 & 0.317921 & 410.3812 & & \\
\hline 0.269968 & 417.084 & 0.268138 & 405.0063 & & \\
\hline 0.219289 & 399.8438 & 0.215699 & 398.9047 & & \\
\hline 0.169053 & 382.2726 & 0.167216 & 392.4108 & & \\
\hline 0.120498 & 363.7943 & 0.121154 & 384.0087 & & \\
\hline 0.071378 & 337.8868 & 0.074138 & 370.6617 & & \\
\hline 0.023502 & 267.8795 & 0.027225 & 326.4956 & & \\
\hline 0.018168 & 252.8002 & 0.019344 & 304.2083 & & \\
\hline
\end{tabular}

